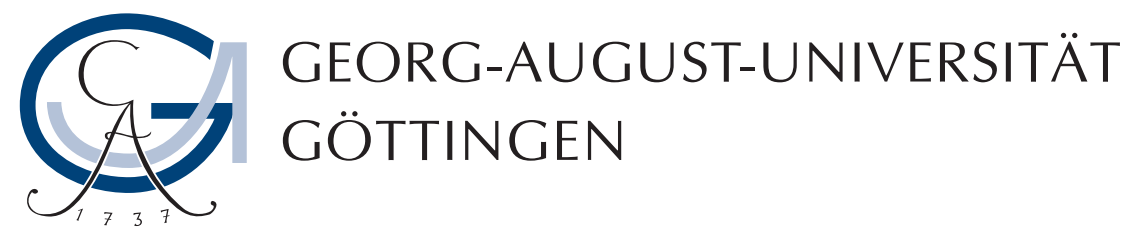

\title{
Aberration correction in STED microscopy
}

\author{
Dissertation \\ for the award of the degree \\ "Doctor rerum naturalium" \\ of the Georg-August-Universität Göttingen
}

within the doctoral program

"Physics of Biological and Complex Systems"

of the Georg-August University School of Science (GAUSS)

submitted by

Joris van Dort

born in Alfeld/Leine, 15.10.1989 


\section{Members of the thesis committee}

Dr. Katrin Willig (Referee)

Nanoscale Microscopy and Molecular Physiology of the Brain,

Max Planck Institute of Experimental Medicine, Göttingen

Prof. Dr. Christoph Schmidt ( $2^{\text {nd }}$ Referee)

Department of Molecular and Cellular Biophysics,

Faculty of Physics, Georg-August University Göttingen

\section{Prof. Dr. Stefan W. Hell}

Department of NanoBiophotonics,

Max Planck Institute for Biophysical Chemistry, Göttingen

\section{Members of the Examination Board}

\section{Prof. Dr. Carolin Wichmann}

Molecular Architecture of Synapses Group,

University Medical Center, Göttingen

\section{Prof. Dr. André Fiala}

Department of Molecular Neurobiology of Behaviour,

Georg-August University Göttingen

\section{Prof. Dr. Ulrich Parlitz}

Biomedical Physics Group,

Max Planck Institute for Dynamics and Self-Organization, Göttingen 
I declare that this thesis was composed by myself and that the work contained herein is my own except where explicitly stated otherwise in the text. Where the work was done in collaboration with others, I have made significant contributions that are clearly indicated throughout the thesis. I further declare that this work has not been submitted for any other degree or professional qualification except as specified.

Göttingen, October 2018

Joris van Dort 


\section{Abstract}

In the last decade, superresolution microscopy techniques became a valuable tool to study sub-cellular structures with a resolution below the diffraction limit. The use of superresolution for brain research requires to super-resolve structures deep within the tissue of the living mouse brain. The only superresolution technique applied in the living mouse brain so far is stimulated emission depletion (STED) microscopy. However, the penetration depth of STED microscopy in the cortex of a living mouse has been limited to a depth of $40 \mu \mathrm{m}$. In this thesis, I studied the use of adaptive optics with the aim of improving the penetration depth of STED microscopy in tissue. Tissue imaging with light microscopy is hampered by light scattering and aberrations induced by the inhomogeneous refractive index of the tissue. The latter can be improved by using adaptive optics to precorrect for the sample induced aberrations. As it is not possible to measure the sample induced aberrations directly, I use a sensorless approach for correction of the three most important aberrations (astigmatism, coma, spherical aberrations) while reducing the number of required images to a minimum. Therefore, I implemented a single correction device (deformable mirror) into a home-build STED microscope to correct all beams at once. Using only the brightest $5 \%$ of the pixels of an image as the metric for the sensorless approach, I optimized the accuracy of the correction and reduced the total number of images required. First experiments have shown that a single deformable mirror together with the novel 5\%-metric can be used to correct for aberrations in $80 \mu \mathrm{m}$ depth of brain tissue.

\section{Zusammenfassung}

Im letzten Jahrzehnt sind hochauflösende Mikroskopietechniken zu einem wertvollen Hilfsmittel für die Untersuchung subzellulärer Strukturen mit einer Aufösung unterhalb der Beugungsgrenze geworden. Die Verwendung von hochauflösender Mikroskopie in der Hirnforschung erfordert es die Strukturen tief im Hirngewebe der lebenden Maus aufzulösen. Die einzige hochauflösende Mikroskopietechnik, die bisher im lebenden Gehirn von Mäusen eingesetzt wurde, ist die STED-Mikroskopie. Die Eindringtiefe der STED-Mikroskopie in den Cortex einer lebenden Maus ist jedoch auf eine Tiefe von $40 \mu \mathrm{m}$ begrenzt. In dieser Arbeit zeige ich, wie man mit Hilfe von adaptiver Optik die Eindringtiefe der STEDMikroskopie in Gewebe verbessern kann. Gewebe mit Lichtmikroskopie zu untersuchen wird durch Lichtstreuung und Aberrationen, welche durch den inhomogenen Brechungsindex des Gewebes entstehen, behindert. Letzteres kann verbessert werden, indem adaptive Optik verwendet wird, um die, durch die Probe induzierten, Aberrationen im Vorhinein zu korrigieren. Da es nicht möglich ist diese Aberrationen direkt zu messen, verwende ich einen sensorlosen Ansatz zur Korrektur der drei wichtigsten Aberrationen (Astigmatismus, Koma, sphärische Aberrationen) und reduziere gleichzeitig die Anzahl der dafür erforderlichen Bilder auf ein Minimum. Dazu setze ich eine adaptive Optik (deformierbarer Spiegel) in einem selbstgebauten STED-Mikroskop ein, um alle Strahlen gleichzeitig zu korrigieren. Lediglich die hellsten 5\% der Pixel eines Bildes werden als Messgröße für den sensorlosen Ansatz genutzt, um die Genauigkeit der Korrektur zu optimieren und die Gesamtzahl der erforderlichen Bilder zu reduzieren. Erste Experimente zeigen, dass ein einzelner deformierbarer Spiegel zusammen mit der neuartigen 5\%-Metrik ausreicht, um Aberrationen in einer Gewebetiefe von $80 \mu \mathrm{m}$ zu korrigieren. 


\section{List of Abbreviations}

a.u.

arbitrary unit

$\mathrm{AO}$

adaptive optics

$\mathrm{AOM}$

acousto optic modulator

APD

avalanche photo diode

CMOS

complementary metal-oxide-semiconductor (sensor)

$\mathrm{DC}$

dichroic mirror

DF

detection filter

$\mathrm{DM}$

deformable mirror

FRET

Förster resonance energy transfer

GFP

green fluorescent protein

GSDIM

ground state depletion followed by individual molecule return

HWP

half wave plate or $\lambda / 2$ plate

ISC

inter system crossing

LCOS

liquid crystal on silicon (SLM)

LTP

long-term potentiation

NA

numerical aperture

OPL

optical path length

PAINT

points-accumulation-for-imaging-in-nanoscale-topography

PALM

photoactivation localization microscopy

PMT

photo multiplier tube

PPP

$\pi$ phase plate

PSD

post synaptic density

PSF

point spread function

QWP

quarter wave plate or $\lambda / 4$ plate

SLM

spatial light modulator

STED

stimulated emission depletion

STORM

stochastic optical reconstruction microscopy

VPP

vortex phase plate

WF Exc

widefield excitation

WFS

wave front sensor 


\section{Contents}

\begin{tabular}{lll}
\hline & Introduction & 1
\end{tabular}

1.1 History of microscopy . . . . . . . . . . . . . . . . . . . . . . . . . 1

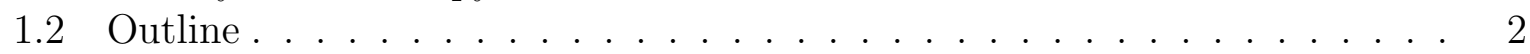

1.3 Fluorescence microscopy $\ldots \ldots \ldots$. . . . . . . . . . . . . . . . . . . . . 3

1.3 .1 STED microscopy $\ldots \ldots \ldots \ldots \ldots \ldots$. . . . . . . . . . . . . . . . . . . . . . . 5

1.3.2 Other superresolution techniques . . . . . . . . . . . . 8

1.3.2.1 Targeted switching microscopy . . . . . . . . . . . . . . 8

1.3.2.2 $\quad$ Stochastic switching superresolution microscopy . . . . . . 9

$1.3 .2 .3 \quad$ MINFLUX $\ldots \ldots \ldots \ldots \ldots$

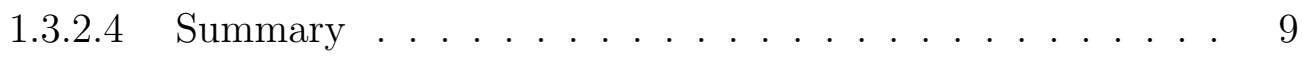

1.4 Wavefront deformations and tools for correction . . . . . . . . . . . . . 10

1.4 .1 Effects of aberrations . . . . . . . . . . . . . . . . . . . 12

1.4 .2 Adaptive elements . . . . . . . . . . . . . . . . . . . . . . 14

1.5 Motivation . . . . . . . . . . . . . . . . . . . . . . 16

2 3D and two-color STED microscopy 18

2.1 Setup design . . . . . . . . . . . . . . . . . . . . . . . . 18

2.2 Setup characteristics . . . . . . . . . . . . . . . . . . . . 21

2.2 .1 Resolution . . . . . . . . . . . . . . . . . . . . . . . . . . . 21

2.2 .2 Crosstalk . . . . . . . . . . . . . . . . . . . . . . . 23

2.3 STED imaging in neuroscience . . . . . . . . . . . . . . . . . 23

2.3 .1 2D two-color STED microscopy of a sciatic nerve . . . . . . . . . 24

$2.3 .2 \quad$ 2D live cell STED microscopy of neuronal actin . . . . . . . . . . . 25

2.3 .3 2D two-color STED microscopy of fixed cultured cells . . . . . . . . 26

2.3 .4 3D STED of the synaptic protein PSD95 . . . . . . . . . . . . . 28

3 Adaptive optics 30

3.1 Zernike polynomials . . . . . . . . . . . . . . . . . . . . . . 30

3.2 Setup design . . . . . . . . . . . . . . . . . . . . . . . . . . . . . . 32

3.2 .1 The deformable mirror . . . . . . . . . . . . . . . . . . 34

$3.2 .2 \quad$ Correcting the PSF movements from aberration modes . . . . . . . 35

3.3 Wavefront optimization $\ldots \ldots \ldots \ldots \ldots \ldots \ldots$

3.3 .1 Different optimization metrics . . . . . . . . . . . . . . . 35

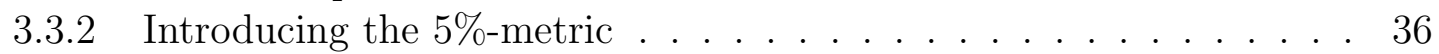

3.3 .3 Optimization algorithm . . . . . . . . . . . . . . . . . . . . 37

3.3 .4 Aberration correction . . . . . . . . . . . . . . . . . . . . . . . 38

3.3.5 Software implementation of the optimization routine . . . . . . . . 42

3.4 Characterization of the deformable mirror . . . . . . . . . . . . . . . 42 
3.5 Correction of brain slice induced aberrations . . . . . . . . . . . . . . . . 44

4 Discussion $\quad \mathbf{5 0}$

4.1 3D and two-color STED microscopy . . . . . . . . . . . . . . . . . . 50

4.2 Adaptive optics $\ldots \ldots \ldots \ldots \ldots \ldots \ldots \ldots$

4.2.1 Correcting the PSF movements from aberration modes . . . . . . . 51

4.2 .2 The adaptive optics setup . . . . . . . . . . . . . 52

4.2 .3 The optimization routine . . . . . . . . . . . . . . . . . . . . 52

4.2 .4 Correction of brain slice induced aberrations . . . . . . . . . . . . 53

4.2 .5 Adaptive optics in in vivo measurements . . . . . . . . . . . . . . . 54

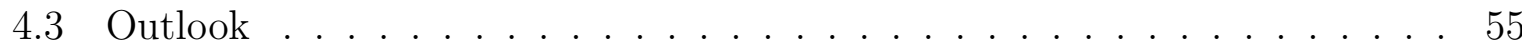

$\begin{array}{lll}5 & \text { Appendix } & 56\end{array}$

\begin{tabular}{ll}
\hline Bibliography & 58
\end{tabular} 


\section{Introduction}

\subsection{History of microscopy}

The usage of optical methods, including microscopy, is nowadays present in nearly all fields of science and often plays a key role in new discoveries. The oldest optical lens has been dated back to around 750BC and is a polished rock-crystal with a slightly convex face. Although it has the optical properties of a lens and could have been used as a magnifying glass, it might just have been created as a decoration [1]. The word "lens" originates from the Latin word of the lentil, which is an edible legume, because they resemble in the shape of its seeds. The Greeks used lenses as burning and magnifying glasses [2] around 400BC. The theoretical description, that light consists of rays that are bundled by the change in refractive index, by using e.g. lenses, was probably not known at this time and the first description dates back to the second century [3]. The potential of lenses to improve vision has been described by many different sources in different parts around the globe [4]. The first combination of single lenses to a more complex optics was probably the telescope. In The Hague, Netherlands, three different men tried to obtain a patent on it in 1608 [5], which the government declined because of its simplicity and the fight over who designed it first. This made the new invention available to everyone and started a hype in the field of optics around Europe. From this time on, the field of astronomy grew quickly into an important research topic. The newly gained possibilities of observation also sparked interest in the observation of smaller scales. Just a couple of decades later, many people from different countries claimed to have built the first microscope [6]. In 1621 Snell's law of refraction was published, which in a simpler form was already published more than 600 years prior in 984 by the Persian Ibn Sahl. The popularity in optics paired with new manufacturing techniques lead to an increase in lens quality. One of the first to describe that white light consists of different colors that get refracted differently and to propose a compound lens that corrects these chromatic aberrations was Sir Isaac Newton [7]. Often the invention of the microscope is closely connected to Antoni van Leeuwenhoek a Dutch scientist. Even though he was not among the first to have discovered the microscope, he still made huge discoveries for his time and is considered as one of the most influential people in early microscopy. He could manufacture lenses with such a high degree of quality, that he was the first to describe to have seen bacteria [8]. Lenses got progressively better over time and with each improvement smaller and smaller structures could be identified. A fundamental discovery in that time was, that this development would not go on infinitely, because light cannot 
be focused beyond a certain spot size. In the 1860's, Carl Zeiss wanted to optimize the empirical production methods that were not compatible with other businesses, so he requested Ernst Abbe to investigate this. Abbe then developed the formula that describes that the diffraction of light limits the resolution of a microscope and is dependent on the wavelength [9, 10]:

$$
d_{\text {lateral }}=\frac{\lambda}{2 n \sin \alpha}
$$

This means, that light of the wavelength $\lambda$ cannot be focused into a spot smaller than $\mathrm{d}$ in the lateral plane, when an objective with numerical aperture $\mathrm{NA}=n \sin \alpha$ is used. Here $n$ stands for the refractive index of the immersion medium and $\alpha$ is the half-opening angle of the objective. In other words, the quality of the lenses is not the only factor that limits resolution, but there is a physically lower boundary in the spot size of light and therefore a natural resolution limit. Having a resolution limit means, that when two objects are closer together than this limit, they appear as a single object in the image. In axial direction the spot size is larger and can be calculated with the following formula [11]:

$$
d_{\mathrm{axial}}=\frac{0.88 \cdot \lambda}{n-\sqrt{n^{2}-\mathrm{NA}^{2}}}
$$

Imaging with $650 \mathrm{~nm}$ and an $1.3 \mathrm{NA}$ objective that uses glycerol as immersion fluid will for example give a resolution of $\sim 250 \mathrm{~nm}$ in lateral direction and $\sim 700 \mathrm{~nm}$ in axial direction. After centuries, superresolution microscopy has recently shown that it is possible to acquire images with a resolution below the diffraction limit and some of these techniques will be discussed in more detail in section 1.3 .2 .

\subsection{Outline}

The following Chapter will give a short overview of the principles that are used in this thesis. These include fluorescence microscopy, STED microscopy in particular, as well as aberrations and their correction with adaptive optics. Chapter 2 will give insight to a twocolor STED microscope with the ability to image with superresolution in three dimensions. The setup was used for various applications to gain more insights into diverse mechanisms and protein localizations in the field of neuroscience. The research about aberrations and their correction is presented in chapter 3. An automated routine to correct aberrations deep in tissue is shown, with the focus on reducing the number of images taken for the correction. Finally the results are discussed in chapter 4, with a short outlook about further applications and ideas for improvements. 


\subsection{Fluorescence microscopy}

The classical light microscope is a wide field microscope, which uses white light of all wavelengths to enlarge an object that cannot be seen with the naked eye. When imaging biological samples, such as cells in a petri dish, it is very difficult to see the cellss. To improve wide field microscopy of transparent samples phase contrast microscopy can be used to make phase shifts visible. These microscopes convert phase differences of the light to amplitude differences, enhancing the contrast and thereby increasing the visibility of the border of the cells [12]. This method improves the visibility of single cells, but the substructure of the cell is still not resolved. To visualize these structures individually, fluorescence microscopy bears a great potential. Here, single components of biological samples can be marked and individually visualized, without observing the unlabeled components of the cell at the same time. It is also possible to perform multi-color labeling, so different structures can be visualized in parallel within the same cell. As the name fluorescence microscopy already implies, the mechanism behind it is called fluorescence. The term was coined by George Gabriel Stokes in 1852 [13], even though the first observations of this phenomenon were performed earlier. A chemical compound that can re-emit light upon excitation is called a fluorophore. The reason that some substances are fluorescent is, that electrons can absorb incoming photons of specific energy by being pushed into a higher energy state. This can be explained using the Jablonski diagram in Figure 1.1. The transition of a valence electron from the ground state to the excited state happens, when a photon with appropriate energy transfers its energy to that electron. There are

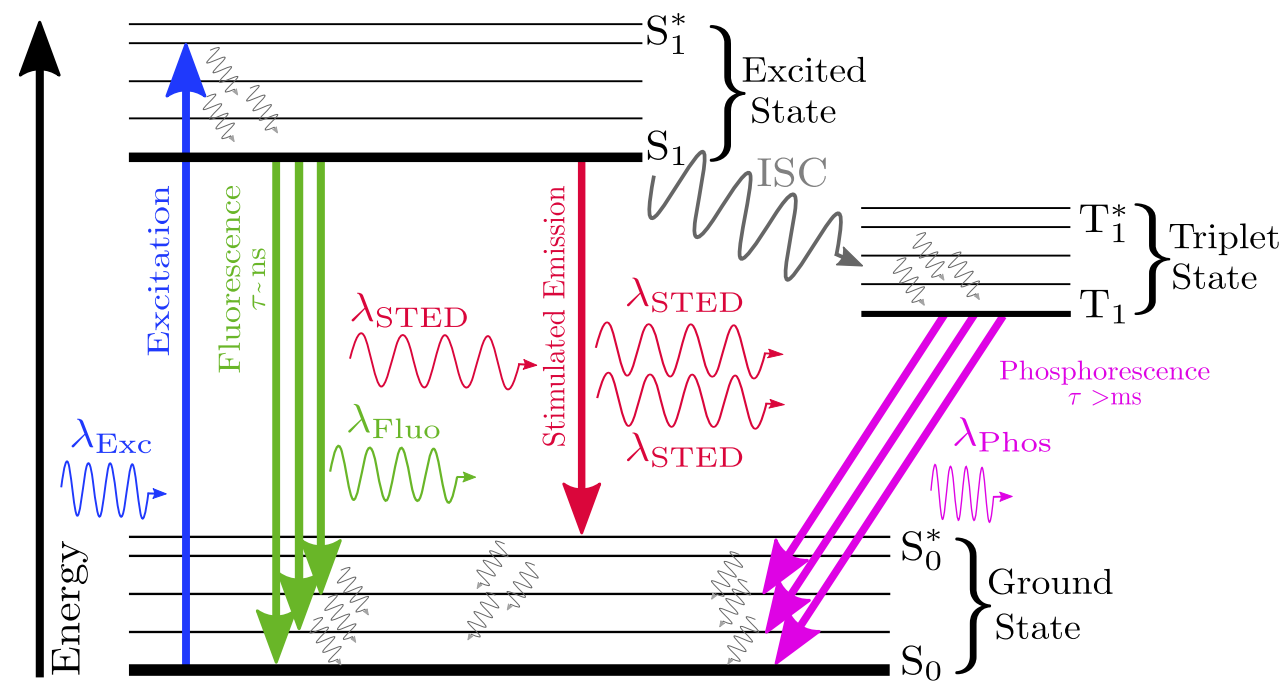

Figure 1.1: Jablonski diagram showing the different energy levels of a fluorophore and the excitation and relaxation of electrons inside. The excitation is shown in blue, fluorescence emission in green, phosphorescence emission in purple and stimulated emission in red. In gray energy loss without emission of photons is shown. 
different possibilities for this excited electron to relax back to the ground state. Firstly, it can relax by emitting a photon of typically lower energy, also called fluorescence or spontaneous emission. This normally happens in the range of nanoseconds after excitation. Secondly, it can be transferred to a more stable triplet state by inter system crossing (ISC). The triplet state is usually energetically lower than the singlet state, which makes the backwards transition unlikely [14]. Spontaneous emission from the triplet state to the ground state is called phosphorescence. The lifetime of the electrons in the triplet state varies from milliseconds to several hours depending on the environmental conditions. Since the mechanism of fluorescence is very complex and the fluorophore is directly interacting with the environment the properties like, lifetime, spectrum etc. can heavily depend on the environment. This can even be used as a sensor, for example for $\mathrm{pH}$-value [15]. The electron might as well react with other molecules and transfer its energy to them, thereby loosing its ability to emit fluorescence. This effect is called photobleaching and is a major concern in fluorescence microscopy. Photobleaching is not fully understood for most molecules, but it is likely that it involves triplet states because they are more reactive. Also oxygen plays a major role, which is why there are anti-photobleaching agents that reduce the amount of reactive oxygen in the sample, to prevent bleaching in fixed samples. Apart from bleaching, another effect can reduce fluorescence, called quenching. This is a reversible effect happening also because of external interactions or internal conversion. Quenching can also be used to measure co-localization of two molecules or to detect structural changes in proteins using Förster Resonance Energy Transfer (FRET) [16]. In FRET, two different fluorescent molecules are needed, a donor and an acceptor. The donor gets excited and will transfer the energy to the acceptor molecule which will emit fluorescence instead of the donor. This can only happen when the molecules are in very close proximity to each other. By observing the ratio between donor and acceptor fluorescence, structural changes in e.g. proteins can be detected [17, 18]. Another option for the electron to directly relax to the ground state is by being stimulated by a photon. This stimulated emission takes place when an incoming photon stimulates the excited electron to emit a photon of the same wavelength as the incoming photon, which is not affected by the stimulation.

Wide field epi-fluorescence microscopy is one of the most used techniques in modern biological research because of its usefulness compared to the simplicity and the cost efficiency. Most setups only require focusing optics as well as a light source, fluorescence filters and a dichroic mirror. It is mostly used when areas of a couple of hundred micron are observed. The problem is however, that the whole specimen is illuminated and therefore unwanted signal from out of focus planes is collected. Blocking the out of focus information can be done by putting a small pinhole in the fluorescence path and collecting the fluorescence with a detector. This small adjustment can improve the image quality by blocking out of focus light but has the disadvantage, that only the light of a single point is collected. To record an image, the beam needs to scan over the area of interest. This is typically done by using a scanning stage on which the specimen is mounted and moved 
in respect to the beam. A faster but more complicated method is to use beam scanning. Here a scanner, consisting of fast moving galvanometric mirrors, is placed in front of the objective to scan the beam over the sample.

\subsubsection{STED microscopy}

STimulated Emission Depletion (STED) microscopy is able to resolve objects that are closer together than the diffraction limit. The theory behind this can be explained by rate equations. Whether an electron is stimulated by an incoming photon or not is a statistical process and can be expressed by the simplified rate equation and its solution:

$$
\begin{aligned}
\frac{\mathrm{d} N}{\mathrm{~d} t} & =-\sigma \cdot I \cdot N(t) \\
N(t) & =N_{0} \cdot e^{-(\sigma \cdot I \cdot t)}
\end{aligned}
$$

Where $N$ is the population of the excited state, $I$ is the intensity of the stimulated radiation and $\sigma$ is the stimulation cross section, which primarily depends on the fluorophore and the wavelength used for stimulation. By solving the differential equation we see, that the population depends exponentially on the laser intensity, the stimulation cross section and the time. The cross section is given in $\mathrm{cm}^{2}$ and is often referred to as the "molecules photon caption area" [19]. The area is roughly proportional to the emission spectrum and greatly depends on the wavelength used for depletion [20]. Because of this, the depletion should be done at a wavelength where the emission is as high as possible for maximum efficiency. This is not possible in STED microscopy, because of possible re-excitation of the molecule with the high intensity laser and therefore, the depletion is done with light shifted to longer wavelength from the excitation maximum.

How stimulated emission can be used to create a superresolution image is sketched in Figure 1.2. Two laser beams are used, where the excitation laser excites the electrons

$$
\text { Excitation Depletion Fluorescence }
$$

Iow STED Power

high STED Power
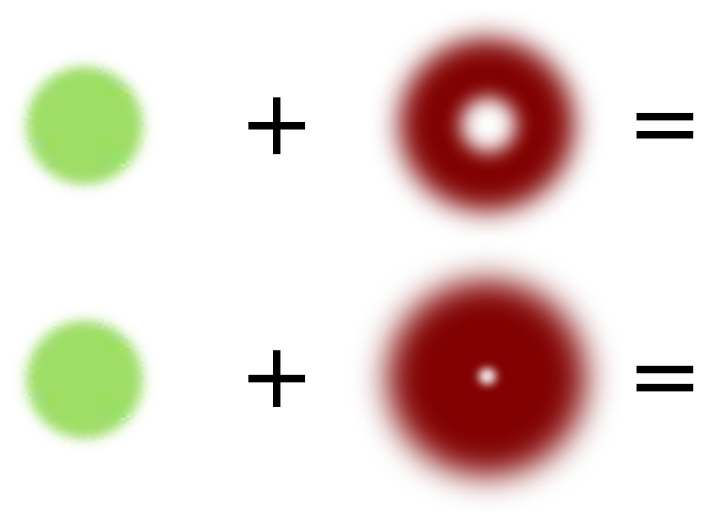

Figure 1.2: Excitation spot and depletion donut create a sub-diffraction limited fluorescence spot. Increasing the STED power leads to a smaller spot size. 
in the diffraction limited spot. This spot is then overlaid with the depletion laser which is formed as a donut with an intensity zero in the middle. Where the intensity of the depletion laser is high, the excited state is depleted, so the fluorescence is quenched at these positions. This creates a sub-diffraction limited fluorescence spot where the depletion laser has zero intensity, because only the excited electrons in the middle of the intensity zero can emit a fluorescence photon. If the power of the STED beam increases, the probability of stimulated emission is higher near the intensity zero and the spot size decreases (Figure 1.3). Harke et al. showed [21], that the resolution is not limited by equation 1.1 anymore but is dependent on the saturation factor:

$$
d_{\mathrm{STED}}=\frac{d_{\mathrm{Abbe}}}{\sqrt{1+d_{\text {Abbe }}^{2} a^{2} \chi}}
$$

Where $d_{\text {STED }}$ is the full width at half maximum (FWHM) of the fluorescence spot and $d_{\text {Abbe }}$ is the FWHM from equation 1.1. Here, $a$ is the pattern steepness in lateral direction and the saturation factor is defined as $\chi=I / I_{S}$, where $I_{S}$ is the effective saturation intensity. It depends on properties of the fluorophore and is the intensity at which the probability of fluorescence emission is reduced by half, so where spontaneous emission and stimulated emission are equally probable. The maximum of the STED intensity distribution $I$ can be increased to decrease the effective fluorescence FWHM as shown in Figure 1.3. With this, there is no theoretical limit to the resolution anymore, assuming infinite STED power. The maximum resolution achieved in lateral direction with STED microscopy is $2.4 \mathrm{~nm}$ on atom-like fluorescent defects in diamonds [22]. A major factor for the imaging quality of a STED microscope is the shape of the depletion beam. For most applications the resolution is maximal in the lateral direction which is achieved by the typical donut shape already shown in Figure 1.2. The beam can be shaped like a donut, by passing through a vortex phase plate (VPP) followed by a $\lambda / 4$-plate (quarter

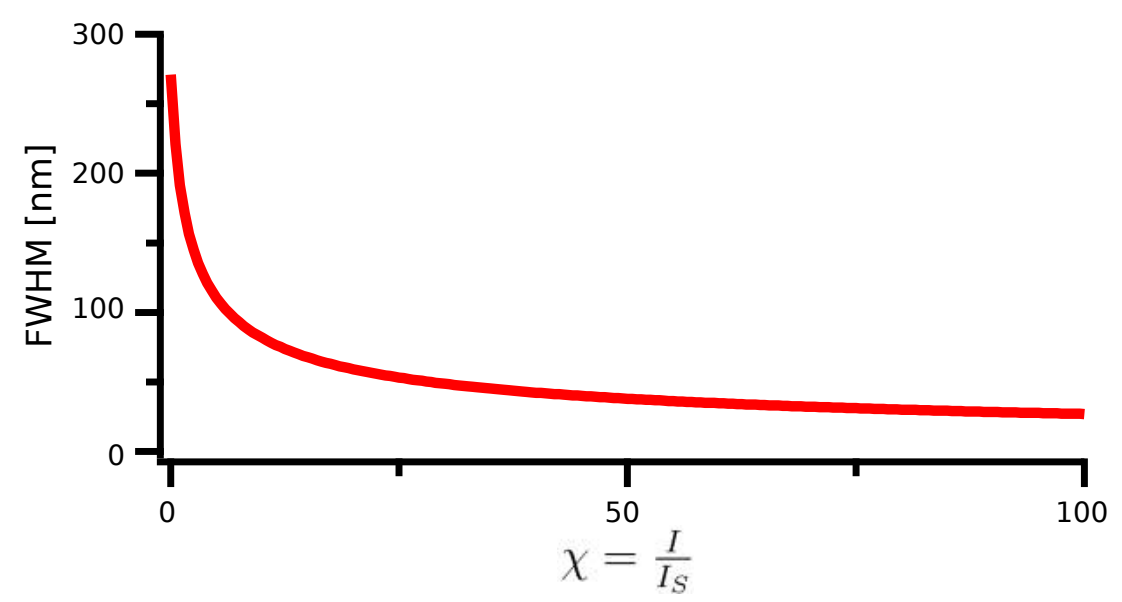

Figure 1.3: Effect of the STED depletion (equation 1.5). Increasing the saturation factor $\chi$ will decrease the FWHM of the fluorescence spot. 
wave plate, QWP) before focusing. The vortex phase plate retards the beam depending on the angular position from 0 to $2 \pi$ which, combined with the circular polarization of the QWP, means that focusing the beam will lead to destructive interference of the light in the focal spot. This leads to a "donut" PSF, with an intensity zero in the middle and a ring around the center. Figure 1.4 shows the point spread functions (PSF) of excitation and depletion beam in the axial direction, as well as the effective area that is fluorescent when a saturation factor of $\chi=15$ is applied. What is additionally shown is a $\pi$ phase
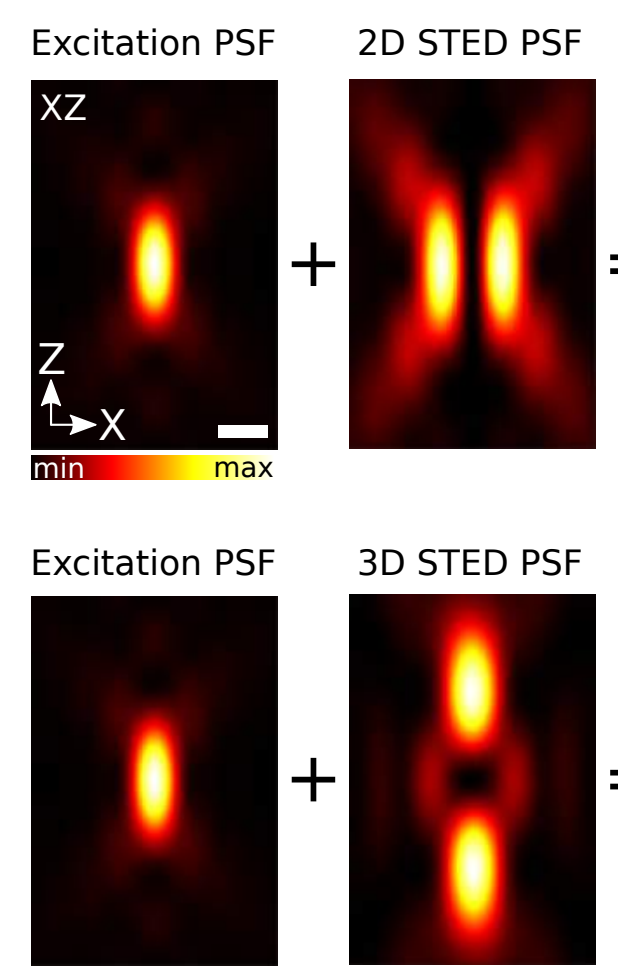
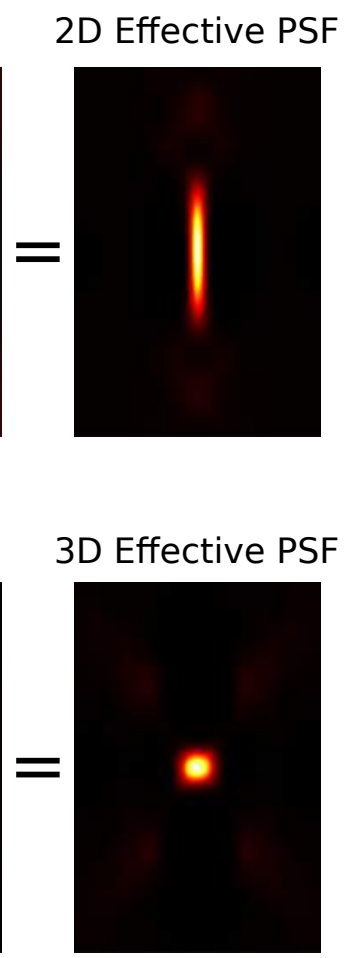
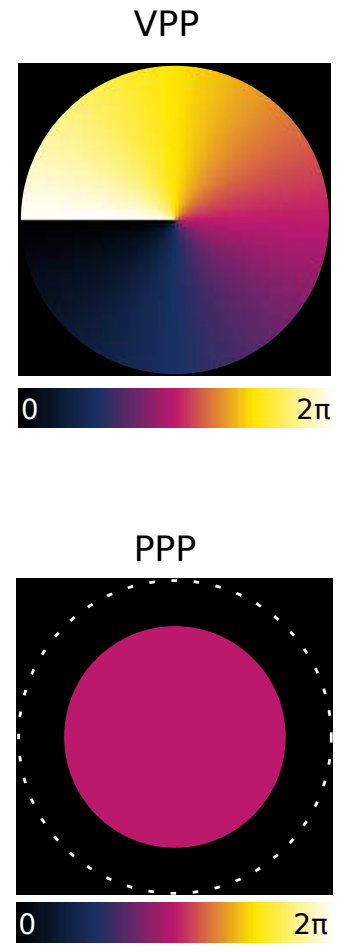

Figure 1.4: XZ simulation of excitation, 2D STED, 3D STED and the respective effective PSF, as well as the VPP and PPP. The saturation factor was set to 15 and the scalebar is $200 \mathrm{~nm}$. In the PPP the dotted line indicates the beam diameter and the $\pi$-phase is applied to exactly $50 \%$ of the area to create the 3D STED PSF.

plate (PPP), that can be used to quench fluorescence in the axial direction. Here the $\pi$ retardation of the middle segment will lead to an intensity zero in the focus, but will stretch the PSF in axial direction in a way that two lobes appear above and below the focus. The shape in lateral direction is still a donut, but most of the intensity will be spread in the upper and lower lobe in axial direction. The difference between the effective PSF created with VPP and PPP is, that the VPP will lead to a superior resolution in vertical direction while not improving resolution in the axial direction. The PPP reduces the fluorescence in the axial direction while only reducing the fluorescent spot in lateral direction by a fraction. Combining the phase plates can lead to superresolution in all three dimensions as described in section 2.2.1. I use pulsed lasers for STED microscopy, where 
the excitation laser emits pulses with a pulse length below 100fs (stretched to picoseconds by a polarizing preserving single-mode fiber) and the STED laser pulses of 700ps. Other than the spatial overlap, the temporal overlap is very important for STED microscopy, since the depletion of the excited state has to happen before the fluorescence is emitted, but after the excitation pulse reached the fluorophore. Since the STED pulse length is shorter than the typical fluorescence lifetime, which is in the range of nanoseconds, the adjustment has to be very precise. The theoretical optimal pulse length of the depletion laser is around 20ps, longer pulses increase the possibility of re-excitation [23]. But in the used STED setup, much longer pulses than that are used because in practice creating short pulses is rather complex. Furthermore, the synchronization of such short pulses is challenging because of jitter and shorter pulses also mean high peak intensities which increase photobleaching [24]. Just as in confocal microscopy a pinhole is used in the detection path and the image is scanned pixel by pixel while the fluorescence is collected for each position.

\subsubsection{Other superresolution techniques}

Apart from using STED microscopy there are other possibilities to achieve better resolutions than normal light microscopy. Since equation 1.1 is dependent on the wavelength of light, using shorter wavelengths will increase the resolution. This is realized in X-ray crystallography as well as electron microscopy and uses wavelength in the single nanometer range to picometer range respectively. These techniques achieve sub-nanometer resolutions, but do not work in vivo. Also, using deconvolution to optimize the image, by estimating the sample structure and considering further information, helps to identify features that are smaller than the actual resolution, but is technically not superresolution. It is also often used to remove imaging artifacts in fluorescence microscopy [25]. In this section, other superresolution techniques will be shortly presented, starting with targeted switching methods.

\subsubsection{Targeted switching microscopy}

STED microscopy is a targeted switching technique and can be seen as a specialized RESOLFT method. In REversible Saturable Optical Linear Fluorescent Transition (RESOLFT) microscopy superresolution is accomplished by switching off unwanted fluorescence in a donut shape. Photoswitchable fluorophores are used, that can be switched on and off using light of a certain wavelength. This switching is done before the fluorophore is excited, so only the fluorophores in the center of the intensity zero of the donut are excited. Compared to STED microscopy, this reduces bleaching effect but the image acquisition takes more time since the switching is slower [26]. 


\subsubsection{Stochastic switching superresolution microscopy}

Instead of scanning an image pixel by pixel with a illumination pattern, it is also possible to use a widefield approach to generate an image with resolution below the diffraction limit. This was shown in PhotoActivation Localization Microscopy (PALM) [27] and (direct) STochastic Optical Reconstruction Microscopy ((d)STORM) [28]. It is realized with special fluorophores that can be activated by light. Separating neighboring fluorophores in time, by illuminating the sample with low activation intensity, so that only a few fluorophores are activated, is the key to this method. This is done stochastically and most of the fluorophores are in a non fluorescent state when the excitation beam excites the fluorescent molecules [29]. The already imaged fluorescent molecules are switched off (e.g. by bleaching them) and further images are taken, starting with the activation of other fluorophores. By doing this, a large number of widefield images are taken with only few fluorescent spots visible, on which the location of single spots can be calculated with sub-diffraction precision. When ideally all fluorophores have been imaged at least once, the image can be reconstructed which leaves a superresolution image that was calculated from widefield images [30]. There are different techniques that use the stochastic switching of fluorophores to localize molecules with a sub-diffraction resolution including Points-Accumulation-for-Imaging-in-Nanoscale-Topography (PAINT) [31] and Ground State Depletion followed by Individual Molecule return microscopy (GSDIM) [32].

\subsubsection{MINFLUX}

A fluorescence technique achieving a resolution of $\sim 1 \mathrm{~nm}$ has been published by Balzarotti et al. in 2017 [33]. It uses stochastic switching combined with patterned excitation beams to localize a single fluorophore with nanometer precision and it has been shown that molecules only $6 \mathrm{~nm}$ apart can be separated. With MINimal emission FLUXes (MINFLUX) it is also possible to track single molecules in E.coli bacteria.

\subsubsection{Summary}

There are a variety of superresolution methods with different strengths and weaknesses and there is an optimal technique for every application. Stochastic superresolution techniques are generally simpler to realize, since a widefield setup is used and not much technical knowledge has to be applied. The downside is that the use is limited to special fluorophores. The sample preparation differs and special buffers, that are not well suited to live cell microscopy, have to be used [34]. Another drawback is the acquisition time which is considerably longer than for other techniques. This is also the drawback when comparing RESOLFT to STED microscopy, because in RESOLFT the activation of the fluorophore is relatively long. Other positive features STED offers are the recording of raw data, a good signal-to-noise ratio and its low demand on sample preparation [22]. This comes at the cost of a relatively complicated setup with advanced optical and tech- 
nical components and photobleaching because of the high power depletion beam. There are different adjustments that can be made to reduce bleaching in STED microscopy to counteract the drawbacks, by intelligently switching off the depletion beam where it is not needed [35, 36]. Still, STED is the most widely used method for live cell and in vivo imaging and has revealed previously unknown detail of biological structure [37, 38, 39, 40], since it has the sectioning capability of a confocal microscope, which PALM and STORM lack. STED microscopy as well as STORM and others also make it possible to image with superresolution in all three dimensions [41, 42, 43].

\subsection{Wavefront deformations and tools for correction}

The first breakthroughs in optical microscopy were possible because the lenses were improved to form a better focus. The quality of optical components improved so far, that it is possible to form diffraction-limited focal spots with standard optical components. A microscope that can achieve the perfect focal spot is referred to as aberration free. However, to get a good quality image the sample itself also should not introduce aberrations. Deviations in coverglass thickness, refractive index of the embedding medium or imaging depth can greatly disturb the focal spot [44], leading to distortions in the wavefront, so called aberrations. Aberrations occur because there is a difference in optical path length (OPL) for each focused ray and this leads to reduced image contrast, brightness and loss of resolution [45]. That aberrations can disturb the image formation is well known and has also been a problem in astronomy in the midst of the last century, due to inhomogeneities and turbulence in the atmosphere. The first person to correct for aberrations in his telescope was Babcock in 1953 [46]. Babcock put a mirror coated with oil into the beam path to change the OPL of the rays. Adjusting the oil surface allowed him to be able to clear up the image. Nowadays, aberration correction is indispensable in astronomy and the correcting elements, which are called adaptive optics (AO), are more versatile [47. The incoming light is reflected off an AO element and projected onto a wavefront sensor (WFS; often a Shack-Hartmann WFS [48]), which divides the wavefront into many subregions with the help of a lenslet array. The ray of each subregion is focused onto a complementary metal-oxide-semiconductor (CMOS) sensor and the positions of the focal spots on the sensor are used to gain information about the curvature in the subregion. By combining the information of each subregion the wavefront can be reconstructed and the AO element can be set to correct this to a flat wavefront [49]. This is often done in a feedback loop, since the aberrations in astronomy are dynamic [50]. In microscopy, especially since superresolution techniques were developed, the quality of the focal spot is of utmost importance and therefore adaptive optics have been implemented in different configurations [51, 52, 53. For STED microscopy, it is worth noting that the resolution scales with the quality of the depletion beam, which is affected more by aberrations than the excitation [54]. In Figure 1.5, it is shown why aberrations occur and how they are 
corrected with adaptive optics. If the refractive index of the medium changes in the focal path, the angle of the light changes which leads to a different position and a difference in OPL between the different rays (not shown here). This leads to an elongated focus if the medium is homogeneous. In Figure 1.5c, the situation is more complex, since biological tissue distorts the light even more. The rays of light take different paths through the sample distorting the focal spot. If the shape of the wavefront and therefore the aberrations are known, the inverted aberrations can be transferred to the incoming wavefront by the use of an adaptive optic element. This is shown in Fig $1.5 \mathrm{~d}$ where the sample cancels out the aberrations given to the wavefront, to form a diffraction limited focal spot. This

a)

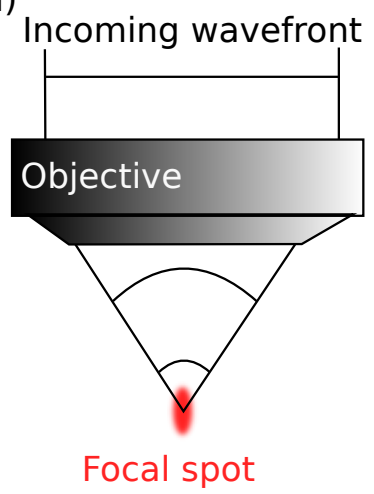

b)

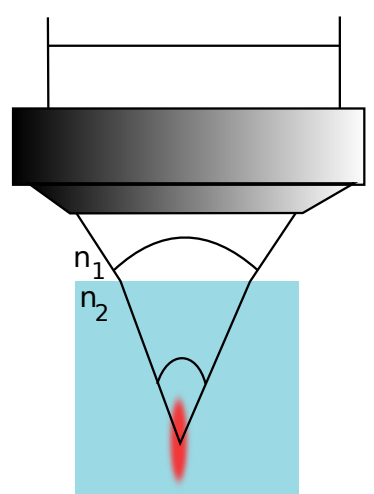

c)

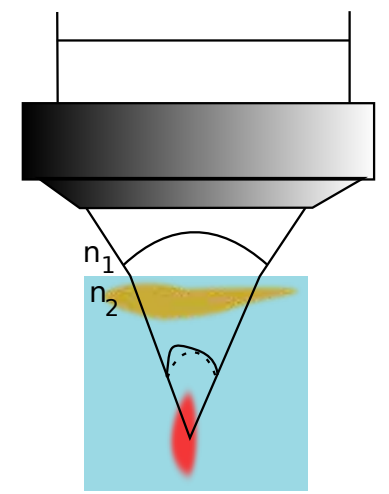

d)

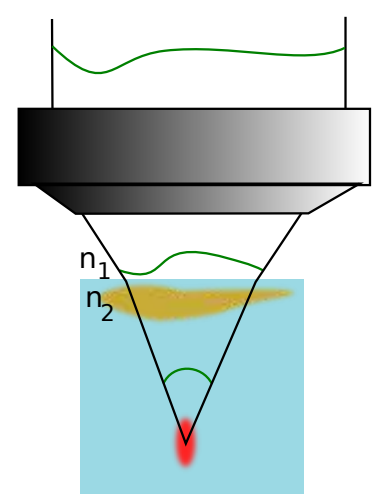

Figure 1.5: Schematics of the creation of the focal spot in different imaging settings. a) Focusing of a flat wavefront with a high-NA objective will create a diffractionlimited sized focal spot. b) Introducing a refractive index change $\left(\mathrm{n}_{1}<\mathrm{n}_{2}\right)$ leads to a spread out focus. c) A complex specimen is added which introduces additional aberrations even though the incoming wavefront is flat. d) The incoming wavefront was adjusted by an adaptive optic element and is not flat. Focusing through the complex specimen corrects the wavefront and creates a diffraction sized focal spot. Adapted from [51].

means that knowing the shape of aberrations makes it also possible to correct for them in deep tissue imaging. The challenge in microscopy is, that in contrast to astronomy, it is not trivial to measure the wavefront distortions directly. The aberrations that are added to the wavefront in microscopy are induced by the specimen itself and extracting the information is challenging. In astronomy a small part of the incoming light is separated and focused onto a WFS to measure the light directly. This is usually done with a guide-star, so the light comes from a point-like emitter and the wavefront is well defined. Using a feedback loop between the sensor and the adaptive optics, the aberrations that are present due to the astronomical turbulence can be corrected in real-time. In microscopy there is no real guide-star present, since fluorescent structures tend to be more complex, which will lead to superpositions of wavefronts coming from different emitters and therefore the wavefront might be misinterpreted. Another limitation is the low light level emitted by the fluorophore which leads to a bad signal-to-noise ratio. Adding a guide-star into a 
fluorescent sample is possible but needs injecting of a different fluorophore which makes it impractical for many applications [55]. The difficulties of direct wavefront sensing in microscopy means that most implementations of adaptive optics are sensorless [56]. This means, that the image can be corrected without directly measuring the wavefront. By selectively inducing aberrations to the system and analyzing the acquired images, the optimal settings of the adaptive element can be found. This sequential imaging is further explained in section 3.3 .

\subsubsection{Effects of aberrations}

It is useful to visualize what effect the single aberrations have on the PSF. In this chapter I focus on the effect of lower order aberrations, which are astigmatism, coma, spherical aberrations and trefoil (see Figure 1.6). Higher order aberrations are not considered in this thesis, because they add only minor distortions to the wavefront. Further implementations are tip, tilt and defocus which are used to position the PSF and have minor effects on the image quality themselves, so they are not presented here. Here I simulated the effect of aberrations of amplitude 0.5rad on excitation and STED PSFs to visualize the changes. The visualization is done using Zernike polynomials $\mathrm{Z}_{n}^{m}$, which are explained in chapter 3.1. Astigmatism $\mathrm{Z}_{2}^{2}$ and $\mathrm{Z}_{2}^{-2}$ only differ in the rotation of the main axis, which is why I only discuss one of each here. The same is true for coma $Z_{3}^{1}$ and $Z_{3}^{-1}$ as well as trefoil $\mathrm{Z}_{3}^{3}$ and $\mathrm{Z}_{3}^{-3}$ (see Table 3.1). The non-aberrated PSFs are the reference to the aberrated ones and they are all symmetric in space and intensity. General observations on the PSFs are, that the maximum intensity in the excitation PSF drops beneath $80 \%$ and that aberrations have more impact of the STED PSF. Astigmatism in an optical setup occurs, when the focal points of the rays coming from perpendicular planes do not meet in the same point. Astigmatism does not appear to affect the excitation PSF largely, apart from the intensity loss (Figure 1.6 b). In the STED PSF the changes are more dominant; the intensity of the usually symmetrical donut shifts to the Y-direction and leaves less light in the $\mathrm{X}$-direction. Studies have shown, that astigmatism is the only aberration that fills the intensity zero of the donut, also quenching the fluorescence in the center $(\sim 1 \%$ at $0.4 \mathrm{rad}$, [57]). This increase in intensity is limiting the brightness of the fluorophore and therefore astigmatism is an aberration which needs to be corrected carefully in order to gain the best imaging capabilities. Coma is an aberration that can be present in optical setups if they are not well aligned, because it occurs when light is passing not perpendicular through a lens. Then, the outer rays of the beam are not focused on the same spot and therefore the intensity is smeared out in one direction. A small shift of the excitation PSF can be seen as well in the XY-PSF, but it is more apparent in the STED beam (Figure 1.6c). Antonello et al. have shown, that this is not just a shift in intensity but also a lateral shift of the intensity zero [57].

The most common specimen induced aberration is spherical aberration, which is also usually the one with the largest amplitude when focusing deep into a sample, because it 


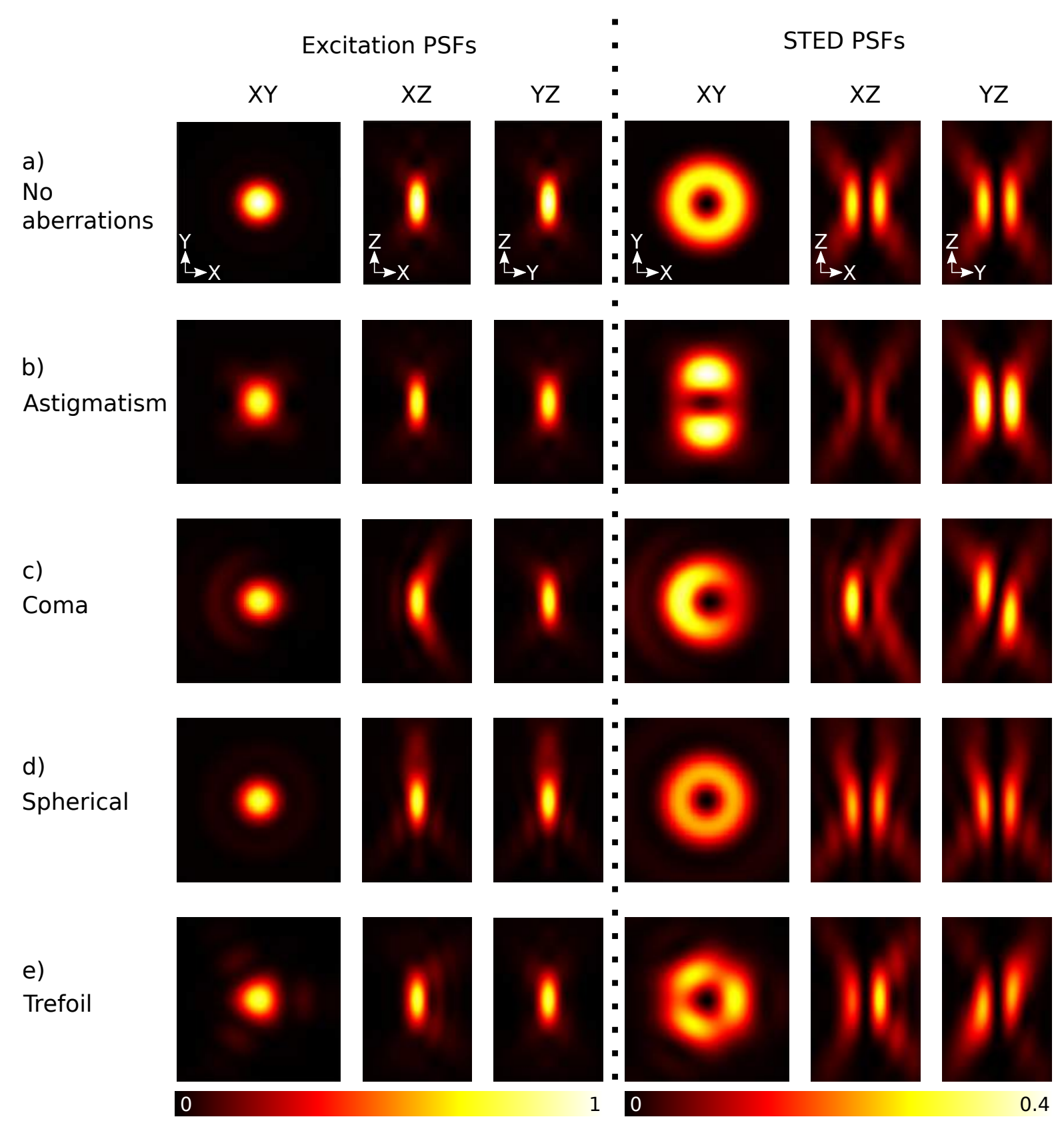

Figure 1.6: Simulated excitation and STED PSFs of different aberrations in XY, XZ and YZ direction. All aberrations presented here have an amplitude of $0.5 \mathrm{rad}$. The colorbar was normalized to 1 for the unaberrated excitation PSF. For the depletion PSFs it is normalized to 0.4, which is the maximum intensity in the STED PSF with astigmatism. The image sizes for XY images are $1.5 \mu \mathrm{m} \times 1.5 \mu \mathrm{m}$ and for $\mathrm{XZ}$ and $\mathrm{YZ} 2 \mu \mathrm{m} \times 3 \mu \mathrm{m}$.

appears when there is a change in the refractive index of the medium. The reason the focal point becomes stretched is, that the rays on the optical axis are focused on a spot closer to the objective than the rays on the outer part of the beam. This stretches the 
focus in axial direction but does not drastically change the appearance in lateral direction (Figure 1.6 d). Because of the more spread out focus, the intensity of the light in the focal plane is reduced. Another aberration is trefoil, which changes the excitation PSF to be a slight triangular shape in XY and leads to a donut that has three intensity maxima on its ring (Figure 1.6. $)$. The general observation that the biggest change in the excitation PSF is the brightness explains why the correction of aberrations is not that crucial in confocal microscopy. In STED microscopy the quality of the donut and its intensity are the most important factors for a good resolution. Because aberrations do affect both factors and astigmatism even fills the intensity zero, the aberration correction of the STED PSF is crucial for a good image quality. Since the intensity loss in the excitation beam impacts confocal as well as STED microscopy, the correction of both beam paths is very important.

\subsubsection{Adaptive elements}

There are different adaptive elements that can shape a wavefront. The most commonly used in microscopy for aberration correction is the correction collar. A correction collar can be found on some high NA objectives to adjust for different coverslip thicknesses or to correct spherical aberrations when focusing deeper into a specimen [58], which makes it a very limited tool for aberration correction. Deformable mirrors (DM) or spatial light modulators (SLM) are often used and are more versatile tools. Deformable mirrors are mainly used for aberration correction, while SLMs have more technological uses and are found in many variations [59, 60]. Both elements are able to shape a wavefront by changing the OPL of the reflected rays (see Figure 1.7). The deformable mirror consists of a continuous reflective surface that is controlled by a number of piezo-electric actuators, that change their height depending on the applied voltage. By pushing or pulling the connected reflective surface, the shape of the mirror changes, which will change the wavefront of the beam that is reflected. On the other hand the layout of liquid-crystal-on-silicon spatial light modulators (LCOS-SLM) is a bit more complex. The SLM consists of multiple layers that are mounted on a silicon substrate. On the silicon there is a layer of liquid crystals, which is a matter that flows like a liquid but has molecules oriented like a crystal. The orientation of these liquid crystals can be controlled pixel by pixel by applying an electric field to an electrode. This orientation change leads to a change in the refractive index that will cause a change in the OPL of the light. For this to work, the light needs to be polarized linearly in the direction of the liquid crystal molecules, else the light will be reflected off the silicon substrate without interaction [61]. The useful thing about polarization dependence is, that with a HWP in front of the SLM the amount of the beam that is going to be altered can be chosen. The drawbacks however are the wavelength dependence and the fact, that unpolarized light (e.g. fluorescence) cannot be corrected. This is different with the DM, since the reflective surface reflects beams of any wavelength and polarization as well as leading to less intensity loss than the SLM. Other differences of the two elements are, that standard commercially available SLMs can have 
more than 10 million pixels (on GAEA-2 from HOLOEYE Photonics, Berlin, Germany) while DMs usually do not have more than a couple of hundred in total. One reason for the low number of pixels on deformable mirrors is, that the surface is not pixelated, but connected and therefore the actuators cannot move freely and independent from each other. This limits correction but makes the changes on the DM smoother. The pixels on the SLM on the other hand can be changed independently of each other, which allows it to be used as a phase mask for STED microscopy. For example the phase jump from 0 to $2 \pi$ in the VPP cannot be displayed by the DM. The SLM can be used to apply a phase plate as well as function as an adaptive optics tool at the same time [62]. The time it takes the device to apply the change to the wavefront differs for both elements. An LCOS-SLM can change its properties up to twenty times a second, which equals a response time of $50 \mathrm{~ms}$, while a deformable mirror can react in time frames faster than

a) Deformable Mirror (DM)

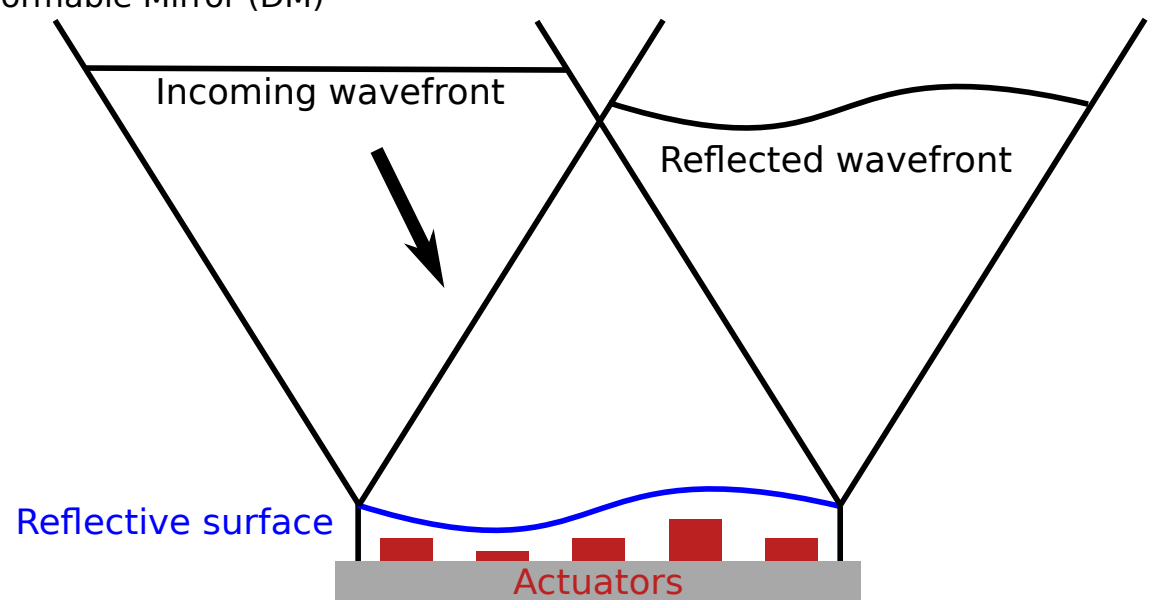

b) Spatial Light Modulator (SLM)

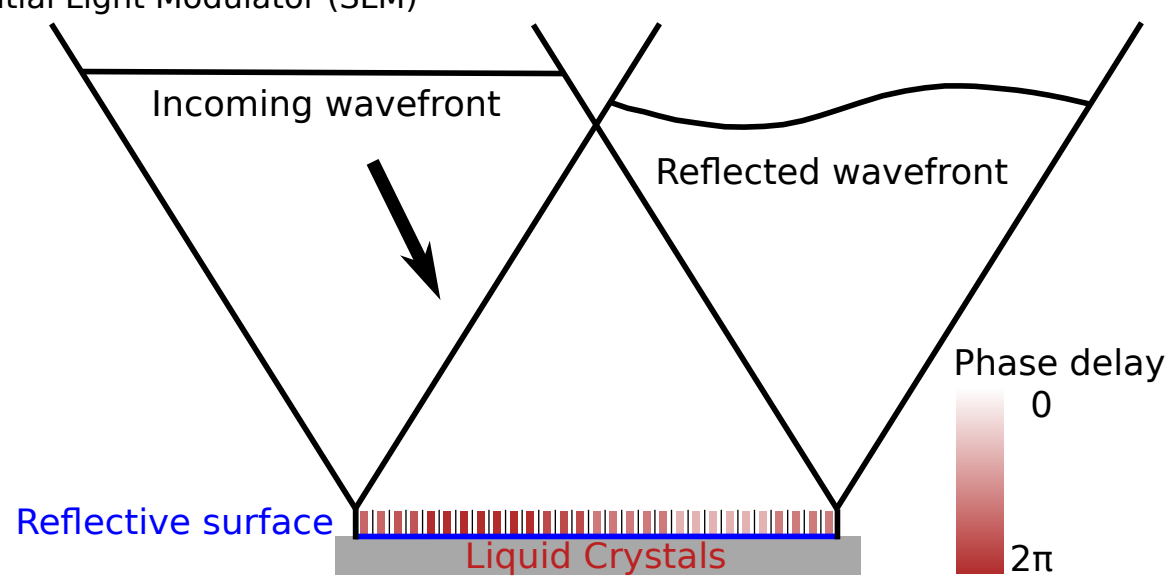

Figure 1.7: Sketch of wavefront shaping with adaptive optics. The reflective surface of the DM can be controlled to reflect the incoming wavefront, introducing a difference in the optical path length. The SLM uses a change in refractive index to achieve this. 
milliseconds [63]. There are different approaches to which element should be used in a STED microscope to correct for aberrations in deep tissue. Some use just one SLM in the depletion path, others use two SLMs for depletion and excitation path [64], while other use both, a DM to correct excitation, depletion and fluorescence emission and an SLM for the depletion phase mask and the more precise, independent correction of the STED beam [65]. Furthermore, adaptive optics can be used to auto-align a STED microscope in lateral and axial direction by applying tip, tilt and defocus to change the position of the depletion beam [66]. It is also possible to focus motionless and keep this focus over a long period of time to prevent sample drift [67].

\subsection{Motivation}

The goal of our group is to image processes in the visual cortex of the mouse brain in vivo, which is why we need to focus light through the brain tissue. To realize the imaging, the bone above the cortex is removed and a window is implanted in the skull of an anesthetized mouse to be able to focus light into the brain. By using transgenic mouse lines expressing fluorescent protein, a subset of cells such as neurons or astrocytes are fluorescently labeled [68]. Imaging in the tissue is currently made possible by using the correction collar of the objective, to reduce the effect of the refractive index change. Although this allows us to correct for spherical aberrations it does not, however optimize the aberrations that are present because of the non-isotropic tissue. Still, the correction collar makes imaging possible with a distance of up to $40 \mu \mathrm{m}$ to the coverslip in the living mouse [69] and $120 \mu \mathrm{m}$ in organotypic brain slices [39]. The optimization of the correction collar during the in vivo experiments takes a lot of time and is not a realistic solution for everyday measurements, as the mouse has to be repositioned every time the collar is turned. Therefore, there is a need for adaptive optics since it can replace the correction collar as the adjustable unit and can correct more precisely by including the correction of more aberrations. This will not only improve the imaging depth significantly, but will also increase imaging speed and is more convenient to use, since the imaging area does not change during the correction. In vivo brain imaging of the mouse adds significant challenges to the usage of adaptive optics, since antibodies, which are typically carrying an organic fluorophore which makes them photostable and bright, cannot be used. Instead we rely on the rather dark and faster bleaching fluorescent proteins (see section 2.3.2). This stresses the importance of sensorless aberration correction with a low number of images. Sensorless approaches used for STED microscopy use up to 150 images per aberration mode (5 applied amplitudes, up to 10 averaged frames per amplitude, repeated two or three times [65]), which is not realistic to be achieved with fluorescent proteins, because of

photobleaching. A process is needed that uses only a small number of images to correct the most basic aberrations. The quality of the optimization is sacrificed for the speed (fewer images) of the optimization and therefore, the conservation of the fluorescence. In 
this thesis, I will show that 5 to 10 images per mode with my approach is sufficient to correct for aberrations, depending on the amplitude of the aberration. To develop the necessary optimization process and test the usability, a STED setup with a deformable mirror was built. The deformable mirror is placed to correct all beams. I chose to optimize the microscope for the red color spectrum, since the red light has several advantages for in vivo imaging. The tissue absorbs less light of this wavelength range, causing less phototoxic stress and reducing background due to the auto-fluorescence of molecules (e.g. hemoglobin [70]). Furthermore, scattering effects are not as severe for longer wavelengths [71, 72]. 


\section{3D and two-color STED microscopy}

Fluorescence microscopy is an outstanding tool in research due to its ability to visualize specific structures. The co-localization of two proteins can be shown by using two spectrally separated fluorescent dyes. With convenient light microscopy co-localization can only be detected with a resolution above $\sim 200 \mathrm{~nm}$, which is the diffraction limit of light. Proteins are much smaller than 200nm and therefore better resolution is needed to check if the co-localization between two proteins really exists. With novel superresolution techniques it is possible to image with a resolution below the diffraction limit and 3D STED microscopy gives us the ability to study interactions with superresolution in all three dimensions $\mathrm{x}, \mathrm{y}$ and $\mathrm{z}$. In this chapter a 3D two-color STED microscope is presented and different applications are shown.

\subsection{Setup design}

This setup was designed similar to [73] for imaging two red emitting fluorophores simultaneously and is sketched in Figure 2.1. For STED, a pulsed laser (Katana 08 HP, OneFive $\mathrm{GmbH}$, Regensdorf, Switzerland) is used, which emits pulses with a frequency of up to $80 \mathrm{MHz}$ and a pulse length of 700ps at a wavelength of $775 \mathrm{~nm}$. By passing through a $\lambda / 2$ waveplate (HWP) and a Glan-Thompson polarizing prism (Bernhard Halle Nachfl., Berlin, Germany) the light is linearly polarized and coupled into a polarization preserving fiber (T2045501-01, Schäfter \& Kirchhoff, Hamburg, Germany). After being collimated, the light passes trough another HWP that controls the polarization and is reflected by a Spatial Light Modulator unit (SLM; Abberior Instruments, Göttingen, Germany). This unit is the main difference to the setup build by Göttfert et al. and consists of an area of pixels whose refractive index can be controlled individually to change the OPL of the light (see section 1.4.2). By changing the refractive index, the optical path length of the beam is altered and the phase of the reflected wavefront can be controlled. Due to the large number of pixels and the individual controlling, complex patterns can be displayed. The beam is reflected twice on different parts of the SLM, so that two separate patterns can be applied. The first one being a helical phase pattern to create a 2D STED PSF and the second one a $\pi$ phase pattern to create a 3D STED PSF (both in Figure 1.4). By rotating the HWP in front of the SLM, the ratio between the two phase patterns can be adjusted. After the SLM, the beam is first expanded by a factor of two and is then merged with the excitation beams by a dichroic mirror (DC4). All beams are reduced in size by a telescope to fit onto the two galvanometric mirrors (MicroMax 673XX, 
Cambridge Technology, Bedford, MA, USA), which act as a beam scanner. The beam is afterwards expanded to cover the aperture of the $1.4 \mathrm{NA}$ objective (HCX PL APO 100x/1.40 OIL STED, Leica Microsystems, Wetzlar, Germany). To ensure an intensity zero in the center of the donut shaped beam, a $\lambda / 4$ waveplate (QWP) is placed in front of the objective, in the inverted microscopy stand (DMi8, Leica Microsystems), creating circular polarization. The objective is moved with a Z-scanning piezo (MIPOS 100PL, piezosystem jena, Jena, Germany) to align the focal plane in axial direction.

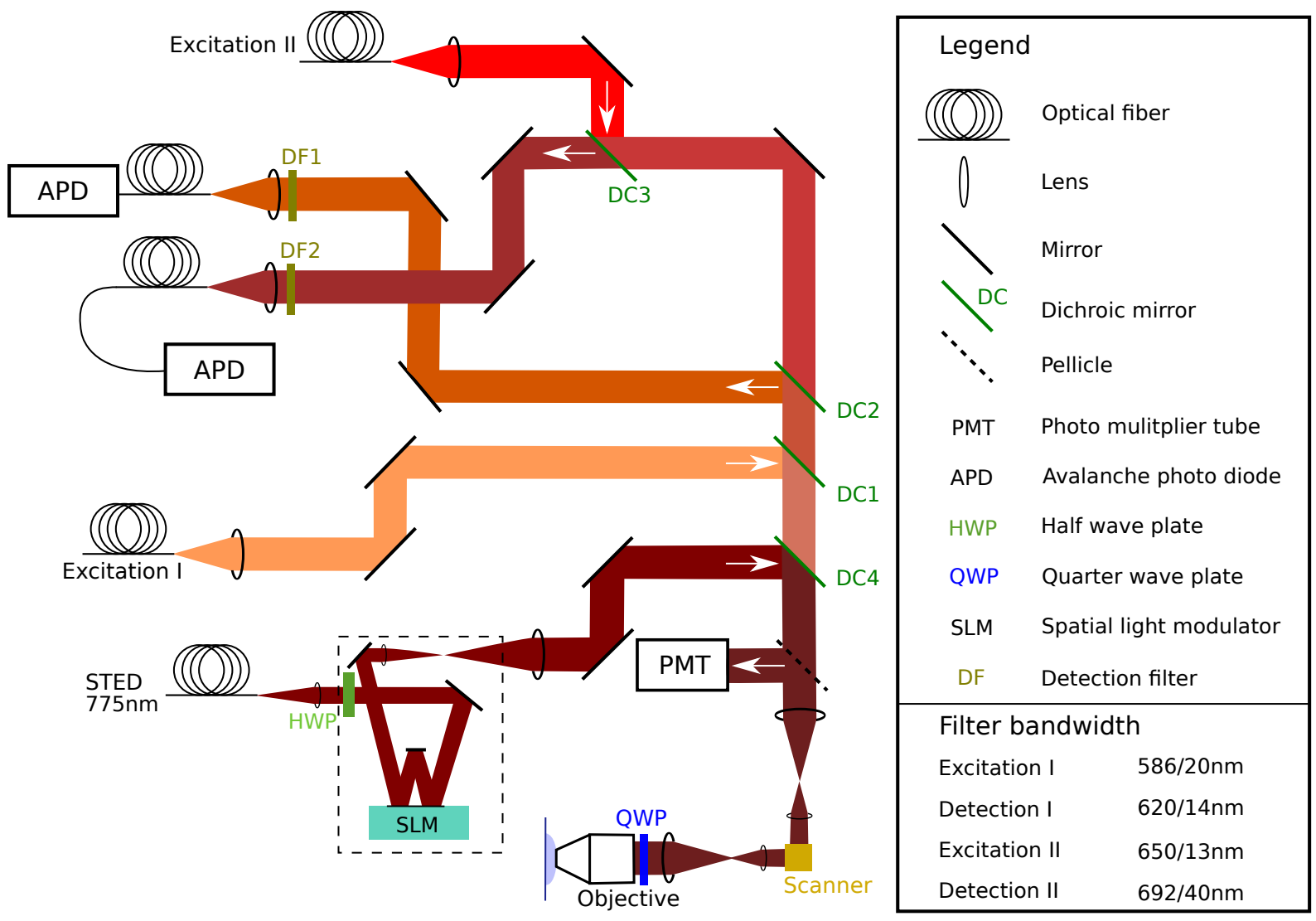

Figure 2.1: Outline of the two-color STED microscope. The pulsed STED laser is reflected onto the SLM and merged with the excitations via a dichroic mirror. All beams are aligned for confocal excitation and detection. The beam is scanned over the sample in the focal plane, while a piezo is moving the objective to scan in the axial direction. The STED donut is visualized by imaging gold beads (150nm gold nanoparticles, BBI Solutions, Crumlin, UK) and detecting the reflection with a PMT. The excitations are also overlaid using this method.

Both excitations (I and II) originate from a Ti:Sa laser (MaiTai; Spectra-Physics, Darmstadt, Germany) which is pulsed at $\sim 80 \mathrm{MHz}$ with a pulse length of below $100 \mathrm{fs}$. From this laser source, white light is generated by passing the light through a supercontinuum device (FemtoWHITE800, NKT photonics, Birkerød, Denmark). The beam is splitted into two by a 50/50 beam splitter. Two bandpass filters (586/20 Bright Line HC, Semrock, Rochester, NY and 650/13 Bright Line HC, Semrock) are used to generate 
two different excitation beams (Exciataion I and II) from the same laser. Acousto-optic modulators (AOM; MT80, AA Opto-electronics, Orsay, France) in each beam path are used to switch the beams on and off within a time frame of 200ns (not shown in Figure 2.1). They are also used to control the intensity. Afterwards the beams are each focused into a 10m long single mode optical fiber (T2045781-01, OZ Optics) to clean up the beam profiles and stretch the spulses. The light is parallelized after the fiber and the beams are combined by two dichroic mirrors (DC1: zt 594 rdc, Chroma, Bellows Falls, VT and DC3: HC BS 622, Semrock). Two other dichroic mirrors (DC2: zt633 rdc, Chroma, DC4: T750SPXRXT, Chroma) are used to separate the descanned fluorescence emission in two spectrally separated detection channels.

The detection belonging to excitation I is cleaned up with a 620/14 Bright Line HC detection filter (DF1; Semrock) and called detection I form now on. The whole path of excitation I and detection I will be called channel I. Accordingly channel II is the path of excitation II and detection II which is cleaned up by the 692/40 Bright Line HC detection filter (DF2; Semrock). After passing the detection filters, the fluorescence is each focused into a $50 \mu \mathrm{m}$ multi mode fiber (M42201, OZ Optics) which acts as a confocal pinhole $(\approx 1$ Airy disc) for the attached avalanche photo diode detectors (APD; SPCM-AQRH-12-TR, Excelitas, Waltham, MA, USA). To adjust and align the PSFs the reflected light of gold beads is detected with a photo multiplier tube (PMT; H10723, Hamamatsu, Bridgewater, NJ, USA). For efficient depletion of the fluorescence, the excitation and STED pulses need to be aligned in space and time. The depletion has to happen right after the fluorophore is excited, but before it can emit spontaneously. If the depletion happens too early it will be inefficient as the fluorophore is still excited by the excitation beam. If it happens to late, some of the excited electrons have already emitted photons and the depletion will also be inefficient. A TTL signal of the MaiTai is used to trigger the STED laser which is delayed by a custom build delay electronics box to adjust the timing between the pulses. The phase delay is adjusted electronically for excitation I and the excitation II beam is synchronized with the first excitation pulse by an optical delay line. To ensure a good spatial co-localization a single STED beam is used to deplete both excitations. Even if the two excitation beams are not aligned perfectly, the zero intensity center of the STED donut will always ensure the co-localization in the STED image.

The excitation and detection bandwidth as well as the spectra of Alexa Fluor 594 (Thermo Fischer Scientific, Darmstadt, Germany) and STAR RED (Abberior, Göttingen, Germany) are presented in Figure 2.2. Alexa Fluor 594 peaks at 590nm and is excited by the MaiTai in the range between $576 \mathrm{~nm}$ and $596 \mathrm{~nm}$, due to the use of the bandpass filter. The emission maximum of Alexa Fluor 594 is at $617 \mathrm{~nm}$, which is the range of the detection filter $(613 \mathrm{~nm}$ to $627 \mathrm{~nm})$. STAR RED has its excitation maximum at $634 \mathrm{~nm}$ which is slightly outside of the excitation bandpass filter of $643 \mathrm{~nm}$ to $657 \mathrm{~nm}$. The emission maximum of STAR RED is at $655 \mathrm{~nm}$ and therefore outside of the detection, which ranges from $672 \mathrm{~nm}$ to $712 \mathrm{~nm}$. As can be seen in Figure 2.1, the $586 \mathrm{~nm}$ excitation (Detection I) will also excite STAR RED to a small degree, but this can be neglected as explained 


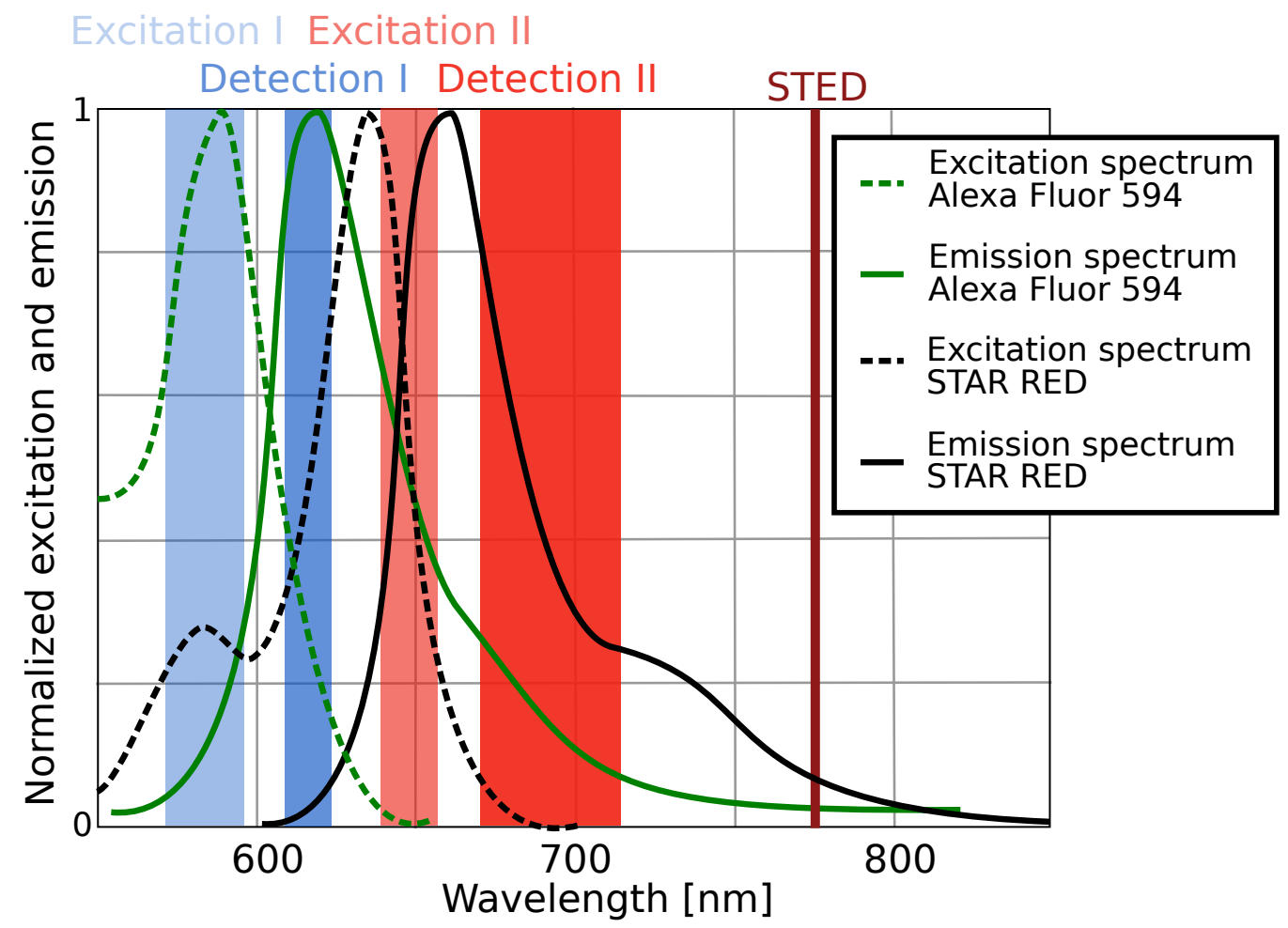

Figure 2.2: Spectra of the dyes and fluorescent filters. In blue, the bandpass filters for excitation I (586/20nm) and detection I (620/14nm) are shown. The corresponding dotted green line and full green line indicate the excitation an emission spectrum of Alexa Fluor 594. Shown in red are excitation II (650/13nm) and detection II $(692 / 40 \mathrm{~nm})$. The black dotted line indicates the excitation spectrum of the dye STAR RED, while the full black line shows its emission spectrum. The STED laser emits at a wavelength of $775 \mathrm{~nm}$, where both dyes are fluorescent.

in section 2.2.2. Detection II (around 692nm) overlaps with the emission of both dyes, so the detector will collect fluorescence of both dyes. The separation of both channels still works, because the detection is separated in time (further see Section 2.2.2). It is important that both dyes emit at the STED wavelength $775 \mathrm{~nm}$, so that the STED beam can deplete the excitations of both dyes.

\subsection{Setup characteristics}

\subsubsection{Resolution}

A superresolution microscope is able to resolve structures below the diffraction limit. The resolution is defined as the minimal distance at which two objects can still be separated. As this would require artificial samples of certain patterns, I prefer to estimate the resolution in the biological sample by measuring the full width at half maximum (FWHM) of 
a) With 2D STED PSF

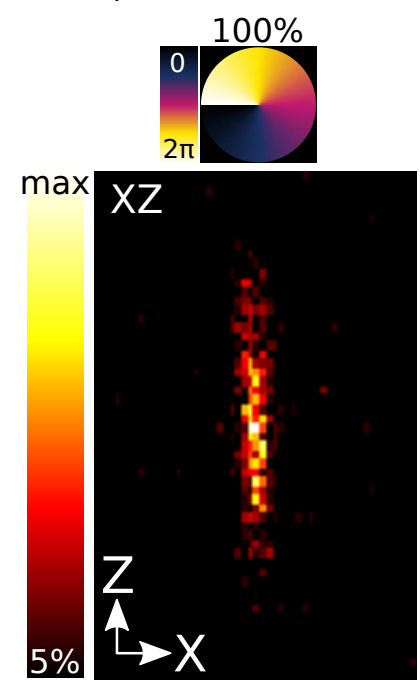

d) Lateral

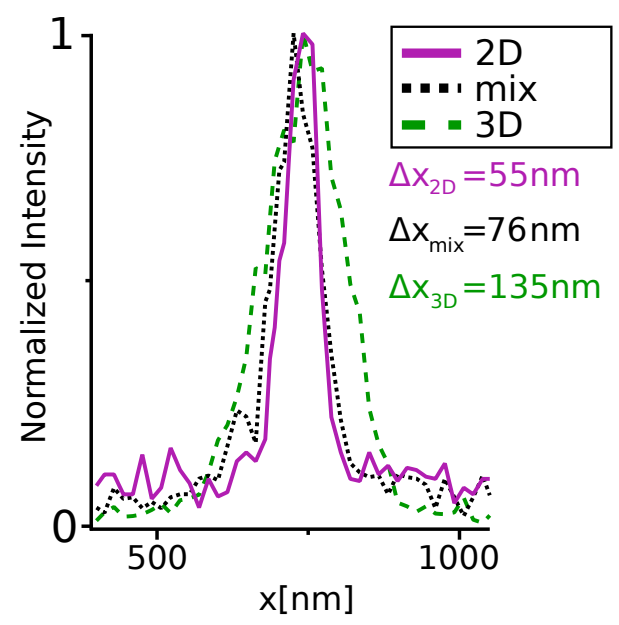

b) With mixed STED PSF
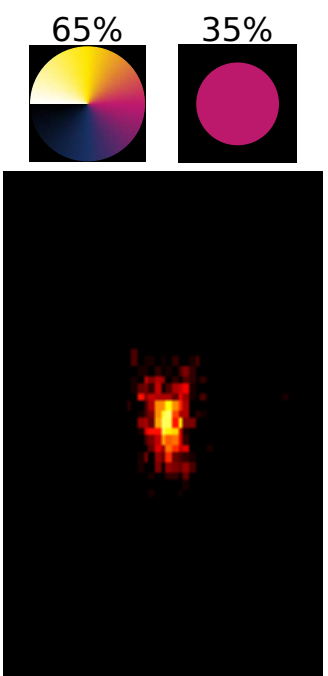

e) Axial c) With 3D STED PSF
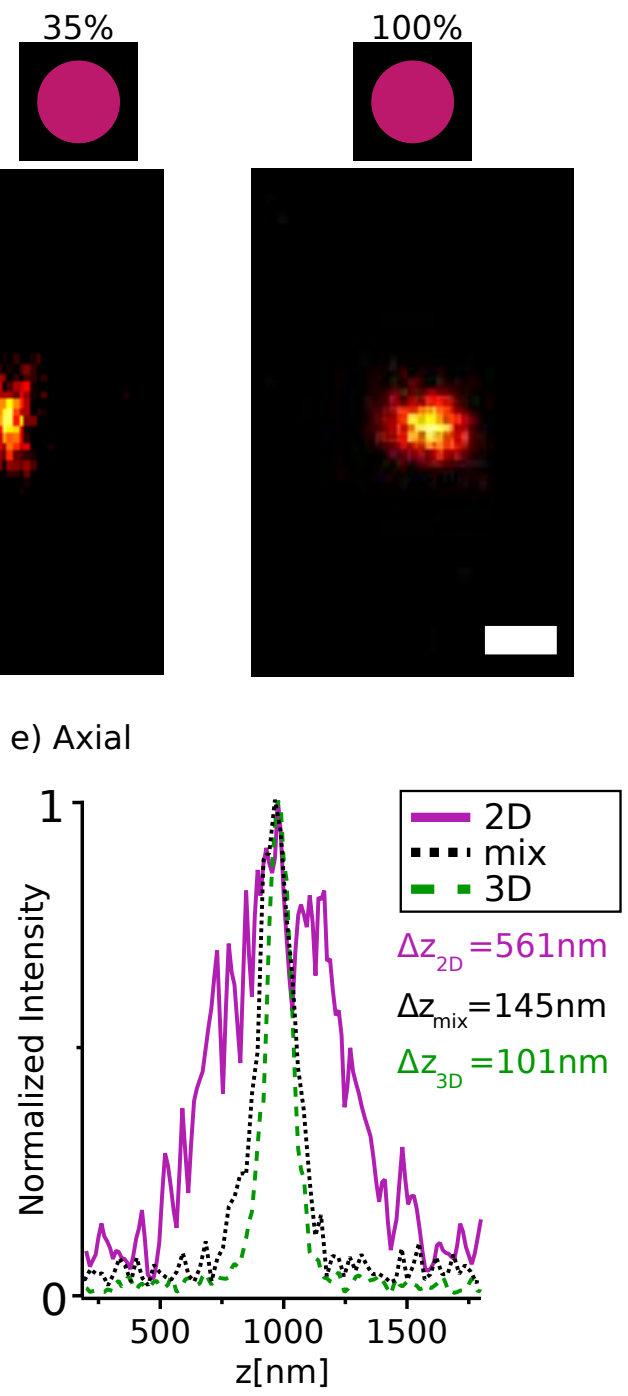

Figure 2.3: XZ-Scans of 40nm fluorescent beads with different depletion settings. a) Scan with the 2D-PSF, b) with a combination (65\% Intensity of the 2D-STED PSF and $35 \%$ intensity of the 3D-STED PSF). c) Scan with the 3D-STED PSF. The effective PSFs have different sizes in lateral $(\mathrm{x})$ and axial $(\mathrm{z})$ direction. In d) the line profiles (width of three pixels) in lateral direction are plotted. The FWHMs are given for all three PSFs. In e) the line profiles are taken in axial direction. Scalebar: 200nm

the smallest point like structures that can be seen, presumably single antibodies. This has been done on the sample presented in Figure 2.6 on various images. Averaging 16 point like structures gives a FWHM for Alexa Fluor 594 of approximately 54nm and a FWHM for STAR RED of approximately $39 \mathrm{~nm}$ at a STED power of $260 \mathrm{~mW}$ in the aperture of the objective. The point like structures that were analyzed are shown in Figure 5.2. The $3 \mathrm{D}$ resolution, so the effect of the $\pi$ phase plate (PPP), was tested on a 40nm fluorescent bead that was imaged in the axial direction with channel II $(650 \mathrm{~nm}$ excitation, $692 \mathrm{~nm}$ 
detection). Figure 2.3 shows STED images of a 40nm bead in the XZ-plane. The images were acquired with a pixel size of $15 \mathrm{~nm}$ in X-direction and $30 \mathrm{~nm}$ in Z-direction and $50 \mu \mathrm{s}$ pixel dwell time. The laser powers were $\sim 10 \mu \mathrm{W}$ for the excitation and $260 \mathrm{~mW}$ for the STED laser.

Imaging with the VPP results in a long thin rod in axial direction (Figure 2.3a) with a lateral FWHM of 55nm (Figure 2.3d) and an axial FWHM of 561nm (Figure 2.3e). Imaging with the PPP (Figure 2.3c), the axial FWHM improves to 101nm and the lateral FWHM to $135 \mathrm{~nm}$ (Figure $2.3 \mathrm{~d}+\mathrm{e}$ ). Taking $65 \%$ of the intensity of the VPP and $35 \%$ intensity of the PPP creates a mixed depletion donut which results in a short rod in axial direction in the STED image [62. This rod has a lateral FWHM of 76nm (Figure 2.3d) and an axial FWHM of $145 \mathrm{~nm}$ (Figure 2.3e) which makes it a better option to image stacks with 3D resolution. By losing image quality in the lateral direction $(55 \mathrm{~nm}$ to $75 \mathrm{~nm}$ ) the resolution in the axial direction can be improved nearly fourfold (561nm to $145 \mathrm{~nm})$. Hence, I can image with superresolution in all three dimensions.

\subsubsection{Crosstalk}

I use a two-color STED microscopy that is operated with just one depletion beam, to ensure the co-localization of both colors in the STED image. Since the stimulated emission cross section is dependent on the wavelength, the two used dyes need to be fluorescent at the depletion wavelength. This also means that the spectra of the dyes are overlapping and therefore are not easy to separate. If just one detector would be used, I could not separate the signal from each other directly, which is why I use two detectors. The imaging is done by scanning each line of the image twice, once with the excitation I and then with the excitation II. When the first excitation is used, only the signal from the respective detector is counted. When the line is scanned with excitation II, detector II collects the fluorescent signal. This will separate the fluorescence of the dyes Alexa Fluor 594 and STAR RED in time, even though their spectra are close together (Figure 2.2). Images of the crosstalk can be seen in Figure 5.1. Here two single color stainings are imaged with

the same excitation power that is used for the two-color image shown in Figure 2.6. It is clear that the crosstalk is below $5 \%$ and is thereby negligible; no further analysis was done.

\subsection{STED imaging in neuroscience}

The described 3D two-color STED microscope was successfully used in several colaboration. We imaged fixed neurons and nerve cells and even performed live cell STED microscopy. The following images were taken as results of some of the colaborations, so the preparation was done by the people mentioned. 


\subsubsection{D two-color STED microscopy of a sciatic nerve}

Nerve structure, composition and conduction velocities are commonly studied properties in regard to an investigation of a potential dysfunction of the peripheral nervous system. The sciatic nerve is the strongest and longest nerve in the human body as well as in mice, which is used as a model system, and has been examined for decades. It consists

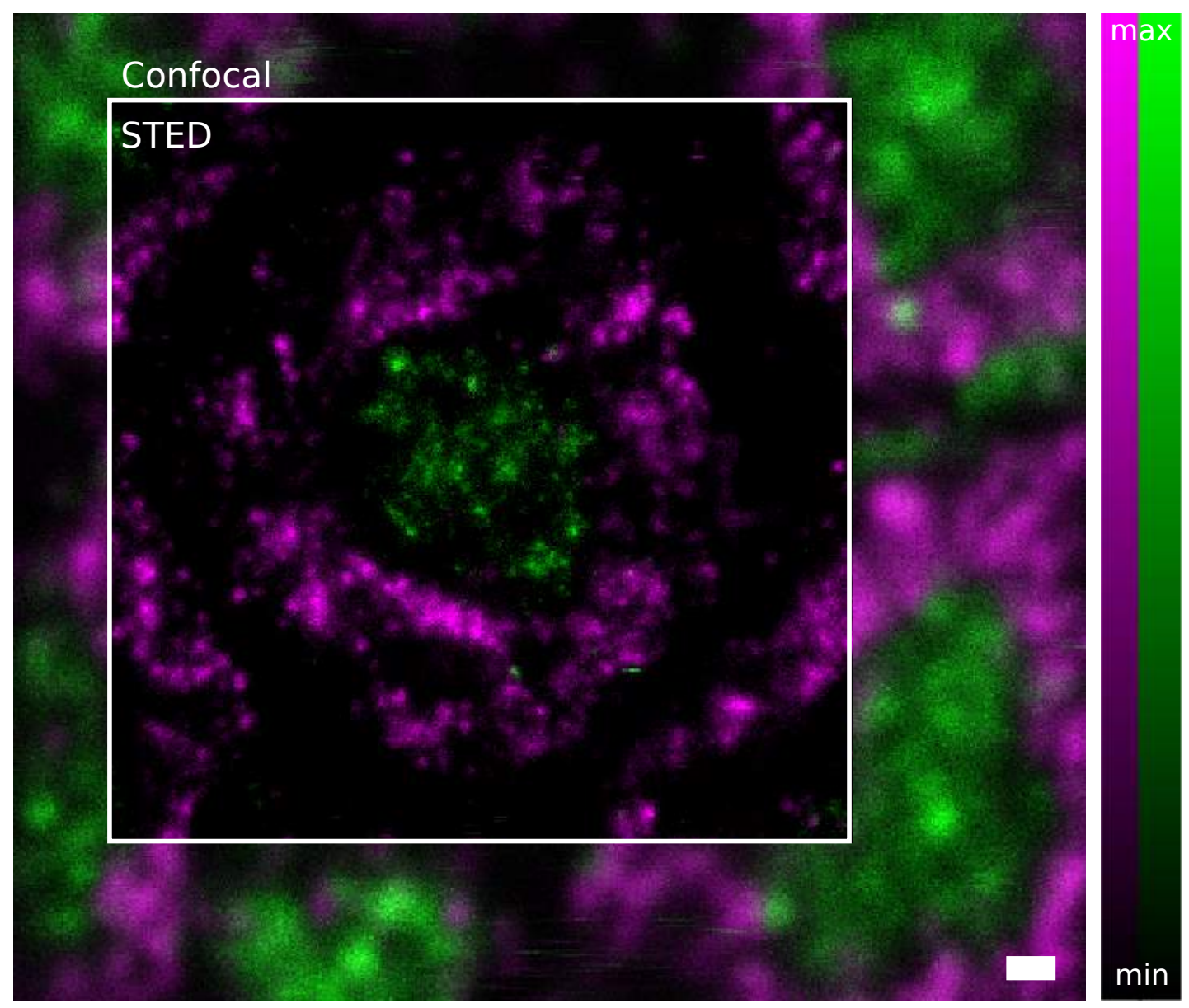

Figure 2.4: Confocal image with STED inset from a sciatic nerve cross section. MBP is shown in magenta (STAR RED) and TM9 is shown in green (Dylight 594). Sample preparation by Meike Hütte. Scalebar: 500nm

of nerve fibers, so called axons, which are enwrapped by myelin sheaths. This nerve insulation by myelin sheath is important, because electrical stimuli can be propagated significantly faster. The main component of myelin is the myelin basic protein (MBP) [74. Superresolution microscopy can help to study this structure further and in greater detail, due to the examination of co-localization between important proteins like MBP and others. The sample shown in Figure 2.4 has been prepared by Meike Hütte (Group of Dr. Manuela Schmidt, MPI of experimental medicine, Göttingen). In brief, sciatic nerves were dissected from legs of mice and embedded in paraffin. Afterwards, $5 \mu \mathrm{m}$ cross sections were produced with a microtome followed by mounting on slides. In Figure 2.4 
the protein TM9 is labeled with STAR RED (shown in magenta; Abberior), whereas MBP is stained with Dylight 594 (shown in green; Thermo Fisher Scientific). TM9 is expressed in the axon and MBP is localized in the surrounding myelin sheath. The images were acquired with a pixel size of $20 \mathrm{~nm}$ and $20 \mu \mathrm{s}$ pixel dwell time. The laser powers in the confocal image were $\sim 8 \mu \mathrm{W}$ for excitation I and $\sim 14 \mu \mathrm{W}$ for excitation II. In the STED image the powers were set to $\sim 15 \mu \mathrm{W}$ for excitation I and $\sim 30 \mu \mathrm{W}$ for excitation II as well as $260 \mathrm{~mW}$ for the STED laser.

\subsubsection{D live cell STED microscopy of neuronal actin}

A big breakthrough in the field of fluorescence microscopy was the discovery of the green fluorescent protein (GFP) from the jellyfish Aequorea victorea (GFP, see [75]). The discovery and development of this protein was groundbreaking and was awarded with the Nobel Prize in Chemistry in 2008. This protein naturally acts as a fluorophore, which makes a fluorescent label redundant. Due to its bright green illumination it is often used as a genetically encoded fusion protein to highlight various proteins or structures. Since the discovery of GFP in the early 1960s, numerous other fluorescent proteins have been discovered or existing ones modified, to broaden the color range from green to blue/cyan (CFP), yellow (YFP), and red (RFP). Red fluorescent proteins, like mNeptune2 are especially important for in vivo imaging because they have several advantages compared to GFP/YFP: The red-shifted excitation light is less absorbed by the tissue which leads to less phototoxicity and tissue autofluorescence of e.g. hemoglobin is reduced, compared to excitation with shorter wavelengths [70]. Furthermore longer wavelengths scatter less in the tissue than shorter ones, which makes them more suitable for in vivo imaging [71]. To find a suitable red fluorescent protein for in vivo imaging, we first tested these proteins in live cell microscopy. To do so, rat primary hippocampal neuronal cell culture of P0-P1 Wistar rats of both sexes have been transduced at 11 days in vitro with adeno-associated viral particles that carried the sequence of Lifeact, an F-actin label fused to mNeputne2, under the human Synapsin1 promoter. After a period of 11 days, transduced neurons were live imaged to investigate the labeled F-actin structure. To image these samples, the setup described above was altered by changing the bandpass filter of detection II to a 708/75nm Brightline (Semrock) filter. After illuminating the sample with excitation I, the signal of both channels was summed up to collect most of the emitted light. This was done because of the broad emission spectrum of mNeptune2, spannig over both detection channels [77]. The image shown in Figure 2.5 shows a bright dendrite with spines, nicely labeled with the red fluorescent protein mNeptune2. The images were acquired with a pixel size of $30 \mathrm{~nm}$ and $25 \mu$ s pixel dwell time. The laser powers were $\sim 2,6 \mu \mathrm{W}$ for the excitation and $117 \mathrm{~mW}$ for the STED laser. The expression of Lifeact-mNeptune2 did not induce any cytotoxic effects [76]. STED imaging revealed actin filaments with an average 83nm FWHM (average of 85, 87,86 and 74nm measured positions indicated in Figure 2.5). Further imaging showed, that the illumination light does not induced phototoxic 
a)

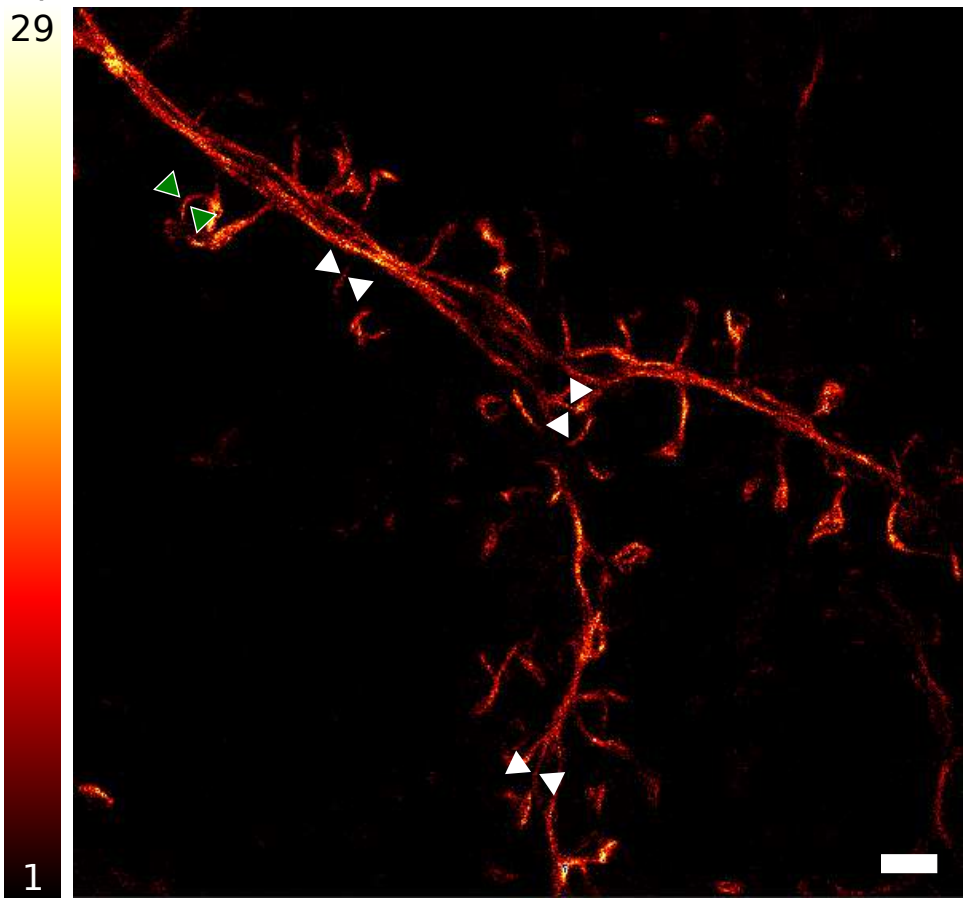

b)

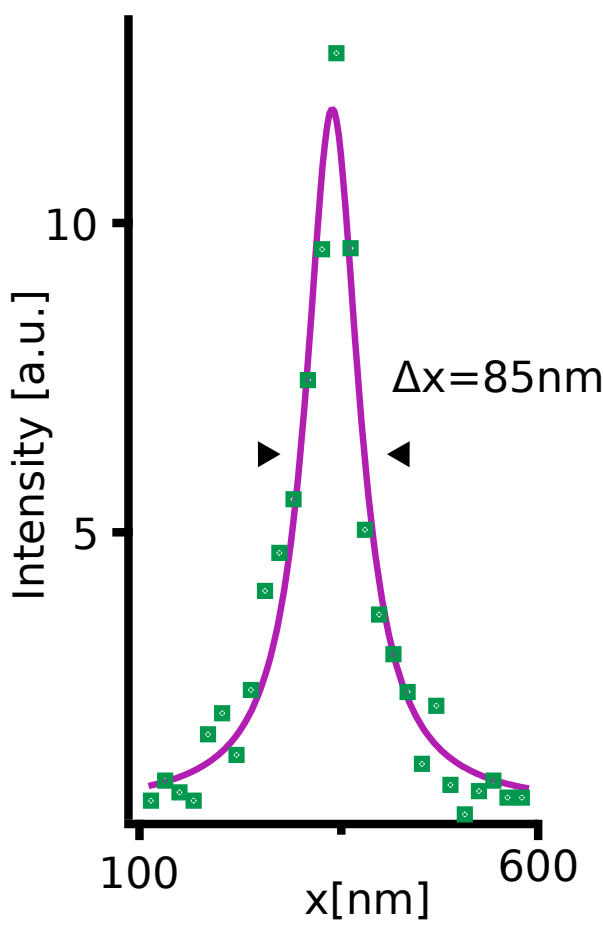

Figure 2.5: STED image of an alive cultured neuron expressing Lifeact, an F-actin label fused to mNeptune2. a) A labeled dendrite with spines can be seen. The green arrow marks the position where the lineprofile (width of three pixels) is taken. b) The lineprofile is plotted with a fitted Lorentzian function which shows a FWHM of $85 \mathrm{~nm}$. The white arrows indicate positions where the FWHM were $86 \mathrm{~nm}, 87 \mathrm{~nm}$ and $74 \mathrm{~nm}$. Image a) adapted from [76]. Scalebar: $2 \mu \mathrm{m}$. Sample preparation by Waja Wegner.

effects, moreover repeated imaging did not change the morphology. This construct was further used for in vivo STED microscopy in our lab [76].

\subsubsection{D two-color STED microscopy of fixed cultured cells}

Brain function is controlled by synapses, which are the fundamental information processing units within the neuronal circuit. On the post-synaptic side of the synapse, we find the Post-Synaptic Density protein 95 (PSD95), a scaffold protein essential for the glutamatergic transmission, synaptic plasticity and long-term potentiation (LTP) [78, 79]. PSD95 stabilizes the post-synaptic ionotropic receptors in the synapse, therefore increasing synaptic strength. On the pre-synaptic part of the synapse, Bassoon is a very large scaffold protein assembled at the active zone, where the neurotransmitters are released to induce the glutamatergic transmission and LTP. To observe the substructure of PSD95 opposite to the active zone after the induction of LTP, we performed an immunostaining of PSD95 and Bassoon on neuronal cells cultured of the hippocampus after a chemical 

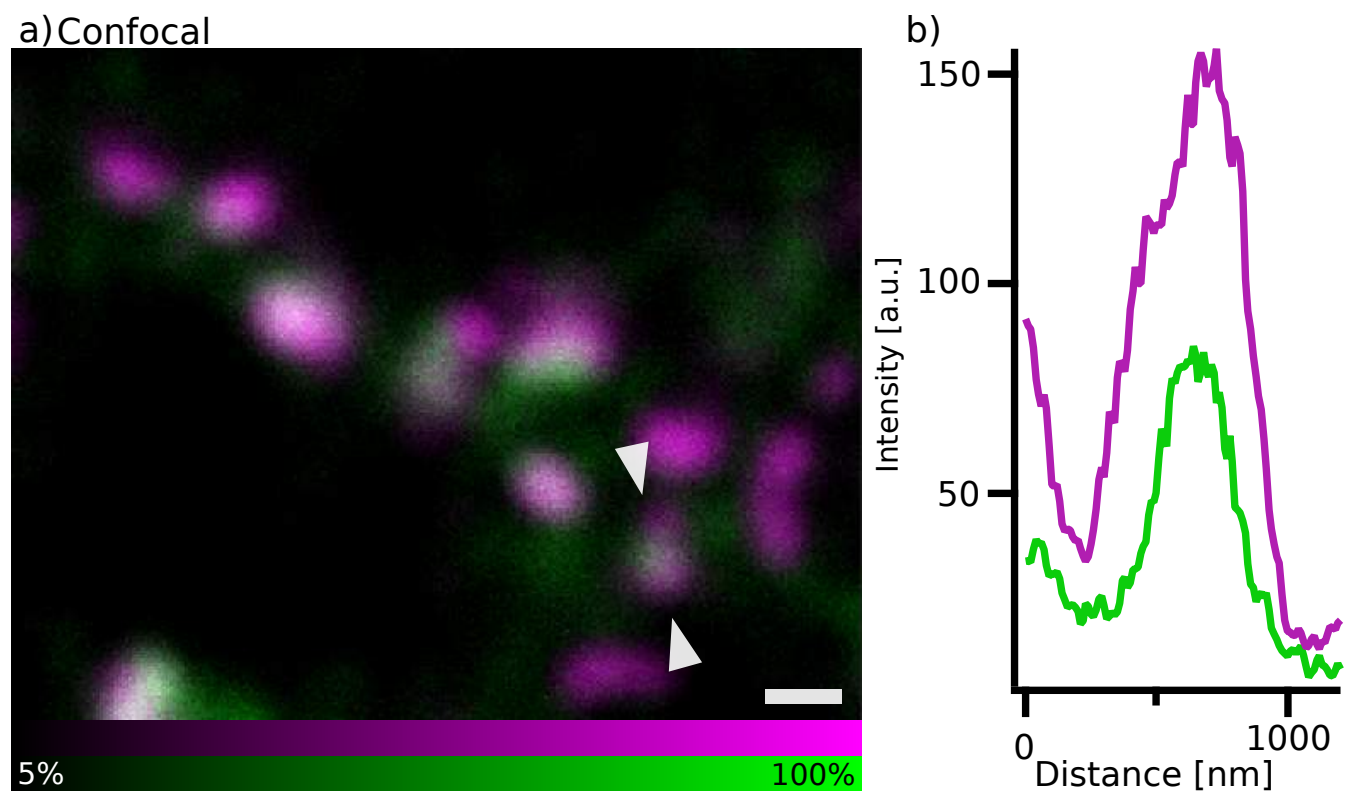

\section{c) STED}
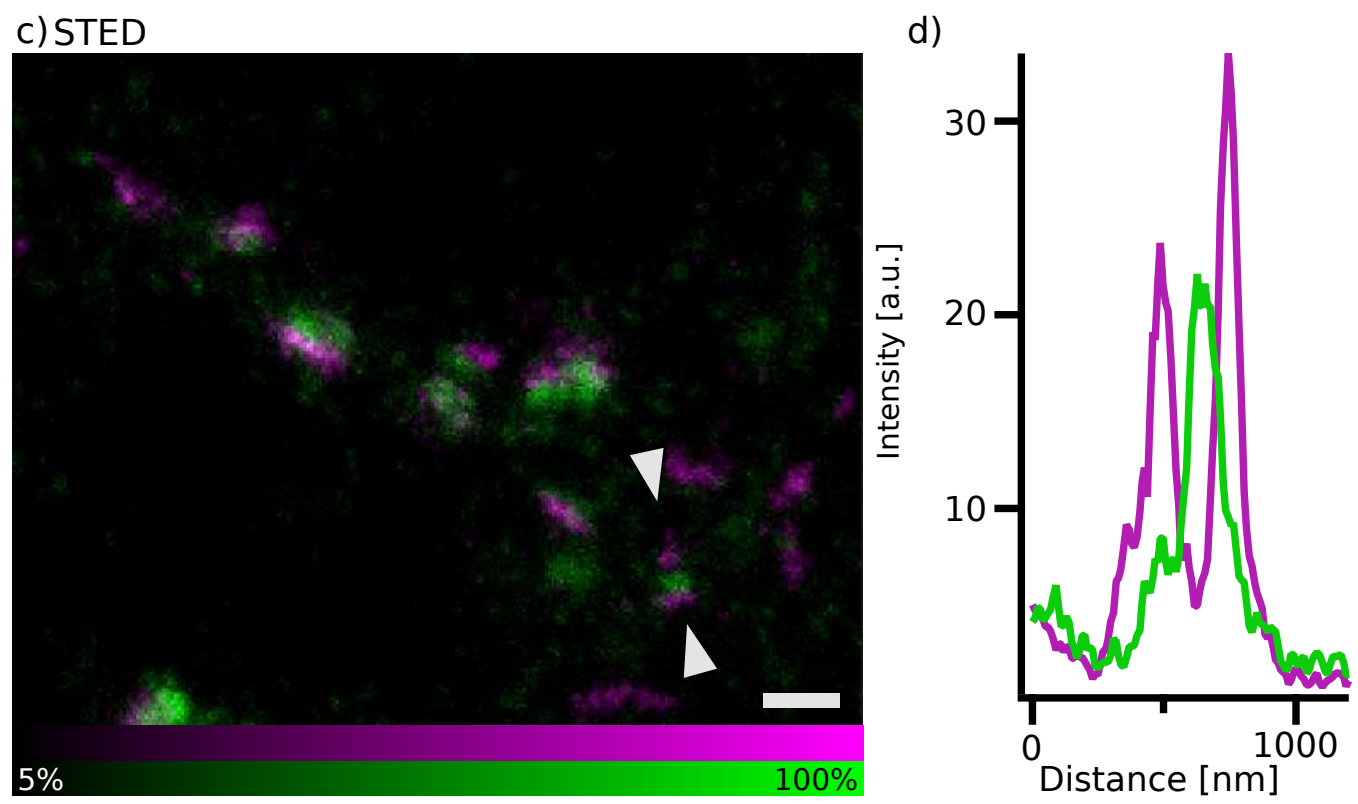

Figure 2.6: Confocal a) and STED image c) of the hippocampal brain cells of a rat with Bassoon colored in magenta and PSD95 in green (raw data). The antibody staining was done with STAR RED for Bassoon and Alexa Fluor 594 for PSD95. The two white arrows indicate where the lineprofiles (width of five pixel) were taken that are plotted in b) and d) respectively. Scalebar: 500nm. Sample preparation by Valérie Clavet-Fournier.

LTP stimulation. We used neuronal cells cultured of 20 days age and fixed them before incubating them with the primary antibodies. The primary antibody against PSD95 (75028, Neuromab) is used in a dilution of $1 / 300$. The antibody against Bassoon (141013, Synaptic System) is used in a dilution of $1 / 500$. The cells are then incubated with the 
secondary antibodies overnight. PSD95 is labeled with Alexa Fluor 594 (shown in green, Thermo Scientific) and Bassoon with STAR RED (shown in magenta, Abberior). The cells are mounted on slides to observe the co-localization of these synaptic proteins with superresolution. Figure 2.6 shows the confocal and STED image of the same area. The images were acquired with a pixel size of $20 \mathrm{~nm}$ and $20 \mu$ s pixel dwell time. The laser powers were $\sim 7 \mu \mathrm{W}$ for excitation I and $\sim 13 \mu \mathrm{W}$ for excitation II. The STED power was $260 \mathrm{~mW}$ in the back aperture of the objective. The better resolution of the STED image is clearly visible. The white arrows indicate where the lineprofiles b) and d) were taken. These were averaged over a width of five pixels. In the confocal lineprofile two large spots can be identified which are in close proximity to each other. The STED lineprofile shows that there are in fact two spots of Bassoon colored with STAR RED and one spot of PSD95 colored Alexa Fluor 594 which is clearly located in between (Figure 2.6). This is an example in which the proteins are well separated which is not visible in the confocal image. The work was done in collaboration with Valérie Clavet-Fournier of our group.

\subsubsection{D STED of the synaptic protein PSD95}

Besides the co-localization of proteins, features like the shape of a structure can also be studied using STED microscopy in more detail. Since these structures are typically three dimensional they appear to have different shapes in a $2 \mathrm{D}$ cut, depending on their orientation. Therefore it is necessary to use a three dimensional approach to visualize the structures in cells and tissue. This can be done using the presented setup and is here shown on the structure of PSD95 assemblies. Electron microscopy showed that the PSD95 appears as an approximately circular disc of $180 \mathrm{~nm}$ to $750 \mathrm{~nm}$ in diameter [80]. The substructure is below the diffraction limit what makes superresolution necessary to observe the structure in greater detail. It has further been observed, that the PSD95 is highly dynamic and appears in different shapes, like macular (homogeneous without interruption) or perforated [81]. To observe this, we stained the Post-Synaptic Density protein 95 with STAR RED in cultured cells. The preparation was similar to the one explained in section 2.3.3 except for the different secondary antibody. To create a 3D reconstruction, the object has to be imaged in all three dimensions which is done by taking a stack of images over the length of one micrometer. The images were then smoothed and processed with the Fiji function "3D project" [82]. The program interpolates the space between the images and reconstructs the three dimensional object (see Figure 2.7). A STED as well as a confocal stack were taken with the same imaging parameters. A STED power of $260 \mathrm{~mW}$ in the aperture was used, with $65 \%$ as $2 \mathrm{D}$ STED PSF and $35 \%$ as 3D STED PSF (see Figure 2.3). The superresolution makes it possible to see a gap in the structure, which is not visible in the confocal image and is only visible at a certain angle. The gap can only be seen in 2D STED if the orientation of the PSD95 assembly is right. In $3 \mathrm{D}$ superresolution it is always visible. The images were acquired with a pixel size of $20 \mathrm{~nm}$ in lateral direction and $50 \mathrm{~nm}$ in axial direction and $50 \mu$ s pixel dwell time. 
The laser powers were $\sim 15 \mu \mathrm{W}$ for excitation and $260 \mathrm{~mW}$ for STED. The work was done in collaboration with Valérie Clavet-Fournier of our group.

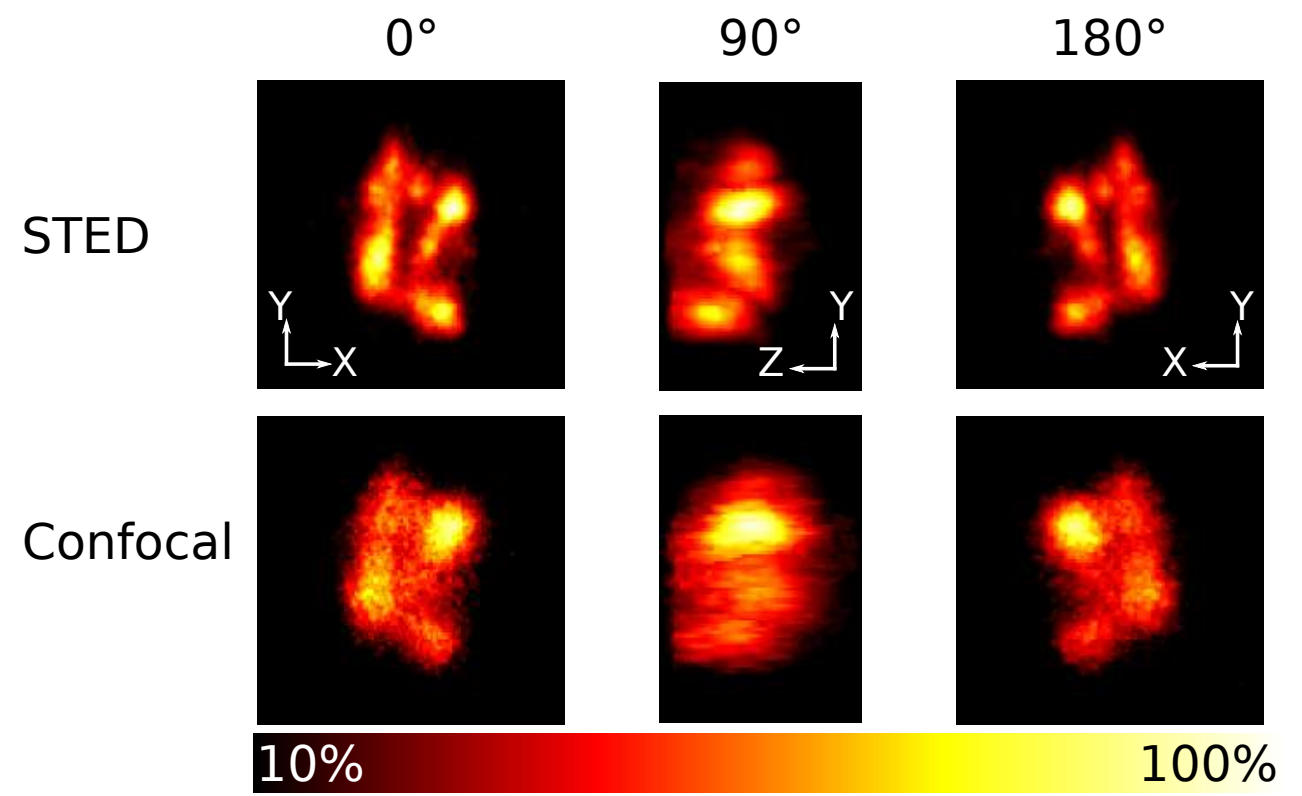

Figure 2.7: 3D STED and 3D confocal reconstruction of a PSD95 assembly. The reconstruction is shown in 3 different angles, rotated around the Y-direction. The images at $0^{\circ}$ and $180^{\circ}$ are of size $1.5 \mu \mathrm{m} \times 1.5 \mu \mathrm{m}$ and the images at $90^{\circ}$ are of size $1.5 \mu \mathrm{m} \times 1 \mu \mathrm{m}$. 


\section{Adaptive optics}

Aberrations are a big concern in deep tissue imaging, especially when superresolution microscopy is applied. The refractive index in tissue is not isotropical because of the composition of lipid membranes, organelles and cytosol which all have different refractive indices and therefore introduce distortions to the focus that need to be corrected. Due to the complex structure it is logical, that I cannot assume that the aberrations are equal over the whole sample. This means it is not possible to apply pre-known corrections in a distinct depth but I need to correct aberrations on the spot to be able to image with superresolution microscopy. Since fluorescent samples are prone to photobleaching and laser light can induce phototoxcitiy in biological samples the number of images taken needs to be reduced to a minimum [83]. In this chapter a STED microscope with a deformable mirror as adaptive element is presented and the improvement of imaging conditions of superresolution microscopy deep into tissue is shown.

\subsection{Zernike polynomials}

In this thesis wavefronts will be described by Zernike polynomials, which were first described by F.Zernike in 1934 [84] and are since then used for adaptive optics [85]. They are orthogonal over the unit circle and the coefficients for single aberrations do not change if additional modes are calculated, which makes them independent. To describe them, polar coordinates are used which are defined as follows:

$$
\begin{aligned}
r & =\sqrt{x^{2}+y^{2}} \quad \text { with } \quad 0 \leq r \leq 1 \\
\theta & =\frac{x}{\sqrt{x^{2}+y^{2}}}
\end{aligned}
$$

The wavefront $\mathrm{W}$ is a sum of single Zernike polynomials $Z$ (also called Zernike modes), with their amplitudes $A$.

$$
W(r, \theta)=\sum_{n, m} A_{n}^{m} Z_{n}^{m}(r, \theta)
$$

where $n \in \mathbb{N}$ is the radial degree of the Zernike polynomial and $m \in \mathbb{Z}$ is the azimuthal degree. The Zernike modes $Z$ are divided into even and odd by differentiating between 
positive and negative value of the azimuthal degree $\mathrm{m}$ :

$$
\begin{aligned}
& \text { even } \quad Z_{n}^{m}(r, \theta)=R_{n}^{m} \cdot r \cos (m \theta) \quad \text { if } m \geq 0 \\
& \text { odd } \quad Z_{n}^{m}(r, \theta)=R_{n}^{m} \cdot r \sin (m \theta) \quad \text { if } m<0
\end{aligned}
$$

The radial function $R_{n}^{m}(r)$ is described as follows:

$$
R_{n}^{m}(r)=\sum_{l=0}^{(n-m) / 2} \frac{(-1)^{l}(n-l) !}{l !\left[\frac{1}{2}(n+m)-l\right] !\left[\frac{1}{2}(n-m)-l\right] !} r^{n-2 l}
$$

The modes are normalized to $R_{n}^{m}(1)=1$ which was introduced by Noll in 1975 [86] to set the root means square (RMS) for every aberration to one, if the amplitude is one. Noll also connected the equations to corresponding names of classical aberrations. These and the normalized equations of the lower order polynomials are shown in Table 3.1 and are visualized in Figure 3.1 .

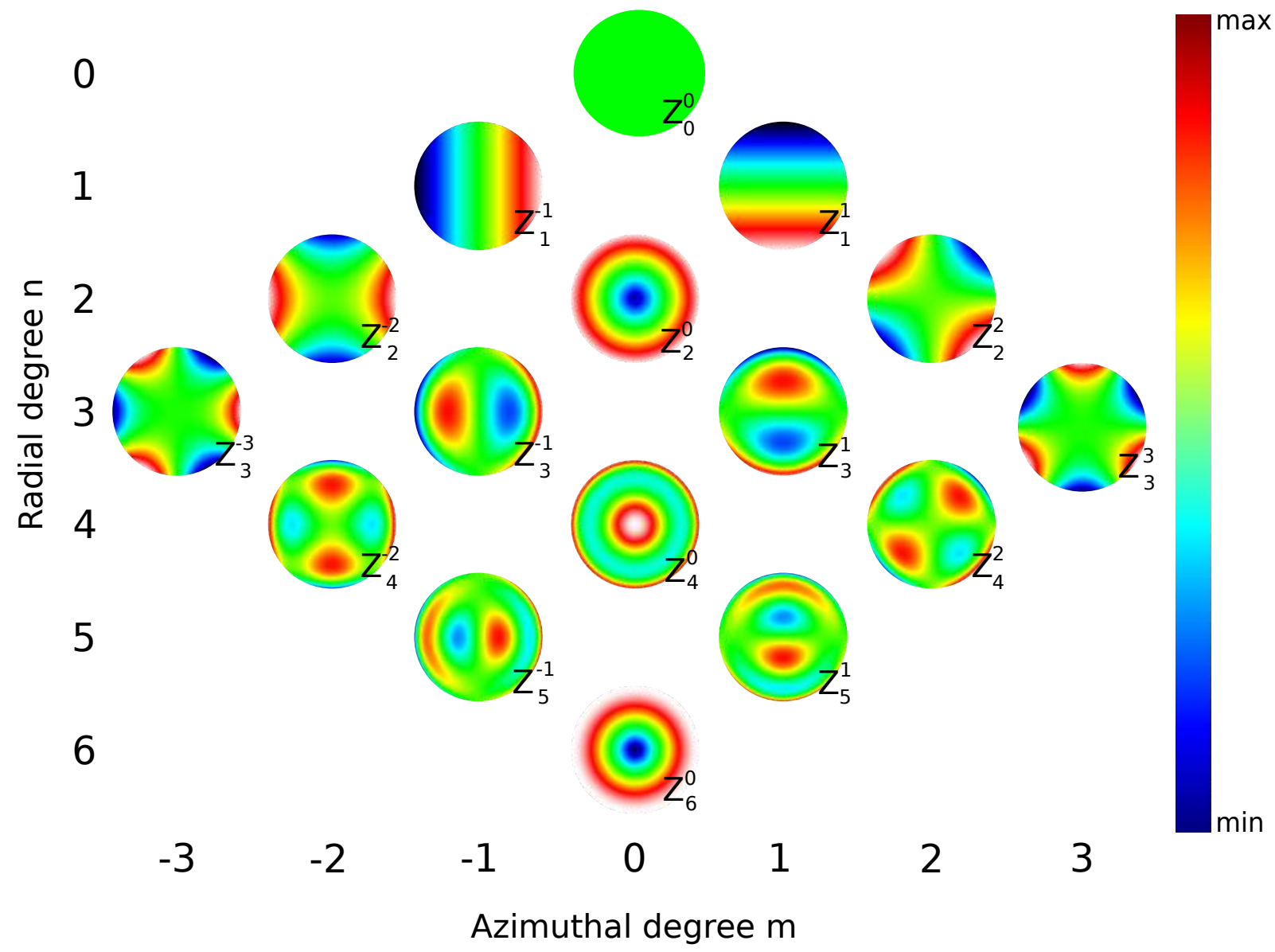

Figure 3.1: A selection of Zernike polynomials. They are ordered by their radial degree $\mathrm{n}$ and their azimuthal degree $\mathrm{m}$ as well as having their mode $Z_{n}^{m}$ written in their lower right corner. 


\begin{tabular}{l|r|r|l|l} 
Index & $\mathrm{n}$ & $\mathrm{m}$ & Normalized equation & Name of aberration \\
\hline $\mathrm{Z}_{0}^{0}$ & 0 & 0 & 1 & piston \\
$\mathrm{Z}_{1}^{1}$ & 1 & 1 & $2 \cdot r \cos (\theta)$ & tip \\
$\mathrm{Z}_{1}^{-1}$ & 1 & -1 & $2 \cdot r \sin (\theta)$ & tilt \\
$\mathrm{Z}_{2}^{0}$ & 2 & 0 & $\sqrt{3} \cdot\left(2 r^{2}-1\right)$ & focus \\
$\mathrm{Z}_{2}^{2}$ & 2 & 2 & $\sqrt{6} \cdot r^{2} \cos (2 \theta)$ & astigmatism at $0^{\circ}$ \\
$\mathrm{Z}_{2}^{-2}$ & 2 & -2 & $\sqrt{6} \cdot r^{2} \sin (2 \theta)$ & astigmatism at $45^{\circ}$ \\
$\mathrm{Z}_{3}^{1}$ & 3 & 1 & $\sqrt{8} \cdot\left(3 r^{2}-2\right) r \cos (\theta)$ & coma at $0^{\circ}$ \\
$\mathrm{Z}_{3}^{-1}$ & 3 & -1 & $\sqrt{8} \cdot\left(3 r^{2}-2\right) r \sin (\theta)$ & coma at $45^{\circ}$ \\
$\mathrm{Z}_{3}^{3}$ & 3 & 3 & $\sqrt{8} \cdot r^{3} \cos (3 \theta)$ & trefoil at $0^{\circ}$ \\
$\mathrm{Z}_{3}^{-3}$ & 3 & -3 & $\sqrt{8} \cdot r^{3} \sin (3 \theta)$ & trefoil at $45^{\circ}$ \\
$\mathrm{Z}_{4}^{0}$ & 4 & 0 & $\sqrt{5} \cdot\left(6 r^{4}-6 r^{2}+1\right)$ & 3rd order spherical \\
$\mathrm{Z}_{4}^{2}$ & 4 & 2 & $\sqrt{10} \cdot\left(4 r^{2}-3\right) r^{2} \cos (2 \theta)$ & 5th order astigmatism at $0^{\circ}$ \\
$\mathrm{Z}_{4}^{-2}$ & 4 & -2 & $\sqrt{10} \cdot\left(4 r^{2}-3\right) r^{2} \sin (2 \theta)$ & 5th order astigmatism at $45^{\circ}$ \\
$\mathrm{Z}_{5}^{1}$ & 5 & 1 & $\sqrt{12} \cdot\left(10 r^{4}-12 r^{2}+3\right) r \cos (\theta)$ & 5th order coma at $0^{\circ}$ \\
$\mathrm{Z}_{5}^{-1}$ & 5 & -1 & $\sqrt{12} \cdot\left(10 r^{4}-12 r^{2}+3\right) r \sin (\theta)$ & 5th order coma at $45^{\circ}$ \\
$\mathrm{Z}_{6}^{0}$ & 6 & 0 & $\sqrt{7} \cdot\left(20 r^{6}-30 r^{4}+12 r^{2}-1\right)$ & 5rd order spherical
\end{tabular}

Table 3.1: The first Zernike polynomials $\mathrm{Z}_{n}^{m}$ and the associated name of the aberration. The first factor of the given equation is the normalization.

\subsection{Setup design}

A STED setup was built to test the capabilities of a DM and to develop and test a routine to optimize the imaging quality automatically. I decided to use a stage scanning setup to simplify the optical path for the initial phase. The outline of the setup is shown in Figure 3.2. The STED beam is coming from the same laser used in the two-color setup described in section 2.1. After being parallelized, the light passes the vortex phase plate (VPP; VPP-1a, RPC Photonics, Rochester, NY, USA) to apply a helical phase delay of $2 \pi$. Afterwards the beam is expanded by a telescope to fit onto the $15 \mathrm{~mm}$ diameter deformable mirror and is reflected off the dichroic mirror (DC1, z780sprcd, Chroma). The size of the beam is reduced by another telescope, to fit into the aperture of the $1.3 \mathrm{NA}$ glycerol objective (HCX PL APO 63x/1.30 GLYC corr 37, Leica). Before the light is focused by the objective, it passes a QWP to ensure the circular polarization that is needed for the donut shape. For the excitation, the output of the fiber of the second channel of the twocolor setup is used, so again white light with a 650/13 fluorescence filter. The excitation is reflected by DC2 (zt633rdc, Chroma) and is also centered onto the DM. Being aligned on top of the STED beam it takes the same path, is reflected off the deformable mirror, gets its diameter reduced and is thereafter focused onto the sample. The epi-fluorescence is taking this exact path back to the DM, passes both dichroic mirrors and is cleaned up spectrally by a filter (708/75 Bright Line HC, Semrock). The fluorescence is focused into a $50 \mu \mathrm{m}$ multi mode fiber that acts as a confocal pinhole of the size of one Airy, where 


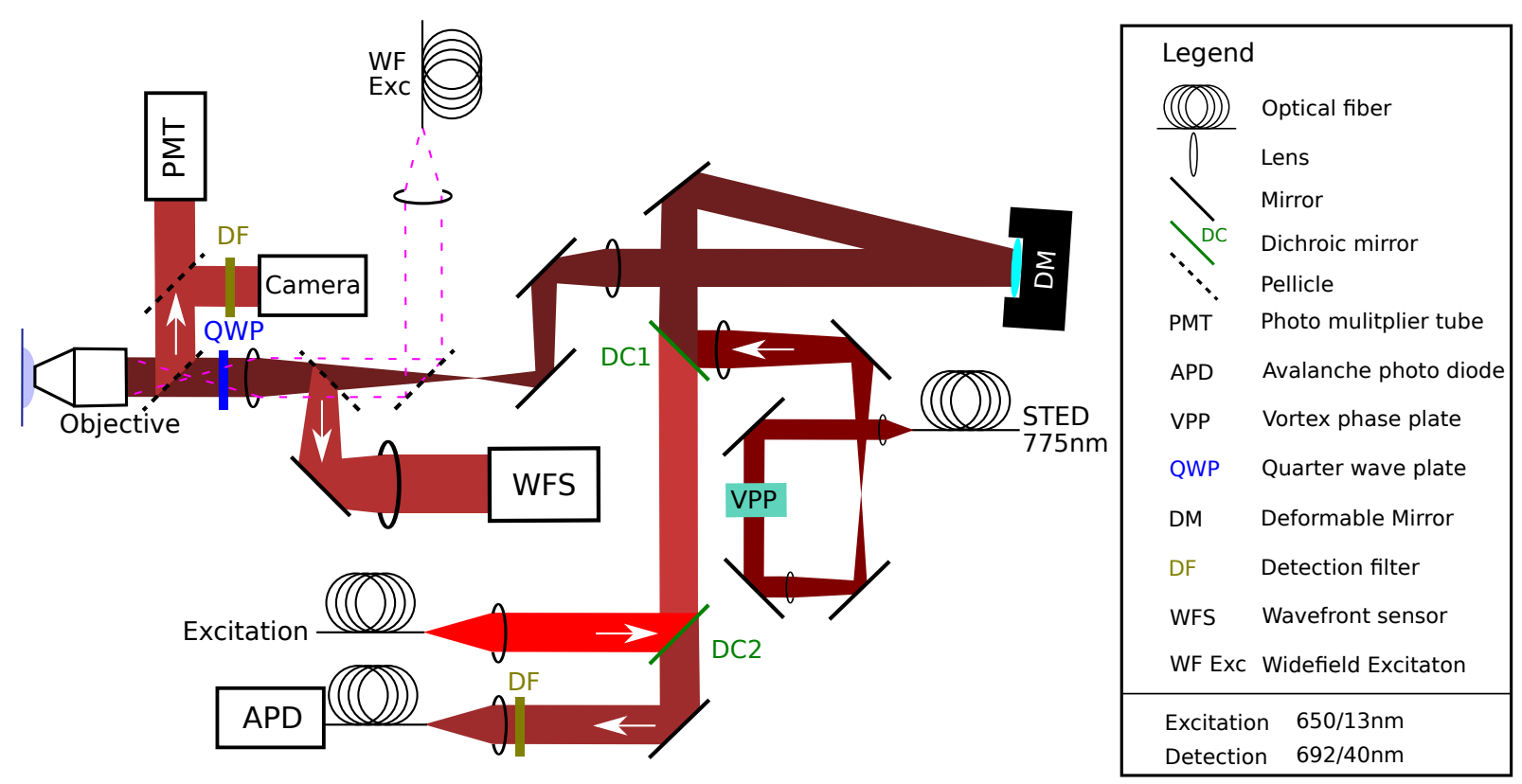

Figure 3.2: Outline of the adaptive optics setup. The 775nm STED laser passes a vortex phase plate (VPP) and is then enlarged to match the area of the deformable mirror (DM). Then it is merged with the excitation (wavelength $653 \mathrm{~nm}$ $657 \mathrm{~nm})$ via a dichroic mirror (DC1) and both are reflected by the DM. The beam diameter is reduced to fit into the glycerol objective. The quarter wave plate (QWP) is required in combination with the VPP to form the donut. The scanning stage moves the sample in all three directions. The emission is separated from the excitation with DC2 and detected by a fiber coupled avalanche photo diode (APD). A 650nm laser diode is used as a wide field excitation (WF Exc) and a camera with a detection filter (FF) will detect the emission. A photo multiplier tube (PMT) is used to adjust the setup. The wave front sensor (WFS) is used to calibrate the DM.

photons are counted with an avalanche photo diode (SPCM-AQRH-12-TR, Excelitas). The sample is moved by a three dimensional scanning stage (NanoMax 300, Thorlabs, Newton, NJ, USA) which is controlled by a piezo controller (BPC 303, Thorlabs). Not having a microscope stand requires a home built wide field fluorescence detection and excitation which is accomplished by using a 635nm laser diode (LDM635/5LJ, Roithner Laser, Vienna, Austria) as wide field excitation, which is focused into the back-aperture of the objective by the lens in front of the objective. The epi-fluorescence is detected with a simple USB widefield camera (DMK 22AUC03, The Imaging Source, Bremen, Germany) with a fluorescence filter (692/40 Bright Line HC, Semrock) in front. As for the two-color setup a PMT (H10723, Hamamatsu) was used to align the beams and check the shape of the STED donut, by detecting the reflected light of $100 \mathrm{~nm}$ gold beads. The wavefront sensor (WFS) is only used for the calibration of the deformable mirror and cannot be used during the experiments. It is important, that the DM, WFS and back aperture of 
the objective are all in conjugate planes to each other. This is achieved through the lens system that also optimizes the beam diameters.

\subsubsection{The deformable mirror}

The deformable mirror used here (DM; mirao 52e, Imagine Optic, Orsay, France) has 52 piezo actuators that cover a pupil of $15 \mathrm{~mm}$ diameter. The surface is coated with silver and therefore all wavelengths and polarizations are reflected. This is important because three beams are reflected by it. The first being the linear polarized STED beam at $775 \mathrm{~nm}$, the second the excitation beam at $650 \mathrm{~nm}$. The unpolarized epi-fluorescence light which has a wavelength ranging from $670 \mathrm{~nm}$ to $710 \mathrm{~nm}$ is also reflected by the DM while traveling to the detection. I assume that dispersion is negligible in biological samples, which means that the optical path length is the same for all wavelengths traveling through the same path. Therefore, the epi-fluorescence needs to be corrected as well, because it passes through the same material as the other two beams and is therefore aberrated. Since the beam needs to be focused into the detection pinhole, aberrations would lead to an imperfect focus and a non-optimal confocal detection. To calibrate the DM a Shack-Hartmann wave front sensor (HASO 3-32, Imagine Optic) is used. The sensor consists of an array of 1280 lenses, which each focuses a small part of the incoming light on a CMOS sensor. This leads to 1280 focal spots on the sensor. The positions of the spots depend on the local phase gradient in the corresponding part of the light. By analyzing the positions the wavefront shape can be reconstructed [87]. Because the wavefront is a sum of independent Zernike polynomials the program can also reconstruct which aberration is present to which extend. The overall divergence of the wavefront from a flat surface is called the RMS error. To calibrate the deformable mirror and have a first estimation of a flat wavefront a silver mirror is put onto the scanning stage and brought to focus. The excitation beam is turned on, reflected off the DM and onto the silver mirror. There it gets reflected onto the WFS and the wavefront can be measured. A routine that is implemented into the Casao software is started, which applies $-0,2 \mathrm{~V}$ and $0,2 \mathrm{~V}$ to it. For each of these measurements the wavefront change is recorded and an interaction matrix is calculated which couples the movement of each actuator to the wavefront change. By inverting the interaction matrix and combining certain elements the program then calculates which actuators to move to apply a certain aberration. These informations are saved in a control matrix and stored for later use. A first instrument flat is calculated by using a feedback loop between the DM and the WFS. Therefore, the measured wavefront is divided into the single aberration modes and the inverted aberrations are applied. This is repeated till the overall RMS error does not improve anymore. 


\subsubsection{Correcting the PSF movements from aberration modes}

Even though the modes are independent from each other, they exhibit a translational movement which complicates the optimization. This is problematic especially when the field of view changes in a measurement. Movements of the PSF are expressed by the aberrations of tip and tilt in the lateral direction and defocus axially. First, the amplitude of tip and tilt is correlated with the displacement measured on gold beads. The twodimensional shifts for astigmatism and coma can be corrected by applying tip and tilt respectively, in addition to the original mode. Hence, the PSF does not move if the aberration is changed. Axial shift is corrected similarly by adding defocus, which is required for spherical aberration. The approach used here is similar to those shown in [88, 89]. The factors for tip, tilt and defocus are always applied to the DM when controlling the aberrations with the Python script and whenever aberrations are mentioned they always refer to these corrected modes.

\subsection{Wavefront optimization}

Due to the difficulty of measuring the aberrations of a sample directly, I choose a sensorless approach to optimize the wavefront. This means, that different amplitudes of aberrations are introduced to the image in order to find the best imaging conditions. For this purpose an image metric is needed, by which the images are rated in respect to each other. This metric is either maximal or minimal when no aberrations are present and is used to describe the quality of the image. I apply different amplitudes of one aberration to the DM, take images and calculate the metric of each amplitude. If I now know how the metric evolves (e.g. quadratic dependence) I can estimate the amplitude for the best image [90]. If this is done with every aberration, the optimal settings of the DM can be found by sequential imaging and without the direct measurement of the wavefront. Different metrics have been used in the past, but since none of these satisfy my needs, I introduce a new one.

\subsubsection{Different optimization metrics}

The metrics that are often used are total image brightness, so the sum of all of pixels of an image, which is as a metric equivalent to the mean value of the pixels [91]. Another metric is the quadratic metric, where the intensities of each pixel are squared and then summed up [56]. Another parameter that can be used to determine the quality of an image is the sharpness. Different approaches to measure the sharpness of the image have been used, e.g. edge, gradient or frequency based measures [92, 93]. It has also been tried to combine these metrics to form a more robust optimization [64]. Unfortunately all of these metrics have drawbacks. The total image brightness has the drawback that background can disturb the optimization, as background is treated equal to signal. The same holds true 
for the square of the pixel intensities, yet the background is reduced due to the fact that the brighter pixels are contributing more. A good signal-to-noise ratio is needed for the image sharpness, since background tends to be sharper than the specimen and therefore being misleading. The metrics have a broad maximum, with the sharpness showing the broadest curve and the quadratic sum of the intensities having the steepest maximum [64]. Having a broad metric means, that its value only changes for severe aberrations and stays nearly constant around the maximum. This means that the single measurements of the metric need to be very precise in order to reliably find the optimal amplitude for the aberration or that large aberrations have to be applied to even see a change in the metric. If the error of a single measurement is larger than the change due to a different metric, many measurements for each amplitude have to be done to have a reliable average. I want to avoid this, by introducing a new metric. The goal of this new metric is to find the optimal amplitude with the fewest images possible. Minimizing the number of images has advantages as being faster, reducing the effect of possible drift of the sample and of course minimizing photo bleaching and phototoxicity in live measurements. The last one being the most important, when imaging fluorescent proteins in vivo. Metrics that have a broad maximum are not well suited, because more images of the same amplitude are needed to get a reliable result. If the total image brightness is used, up to 150 images are necessary to reliably determine the value of one aberration [65]. This renders the use of adaptive optics really difficult or even impossible in the living mouse, when fluorescent proteins are used. The preferred metric has a sharp optimum, so noise in a single measurement is less disturbing and therefore the measurements will be more reliable.

\subsubsection{Introducing the 5\%-metric}

I propose to only sum up the brightest $5 \%$ of the pixels and use this sum as my metric. The quality of this was analyzed with the following measurement. I imaged the same bead and analyzed the total sum of all pixels as well as the sum of the $5 \%$ brightest pixels with differing amplitudes of astigmatism $\left(Z_{2}^{2}\right.$, Figure 3.3). Here the same gold bead has been imaged with differing amplitudes of astigmatism $\left(Z_{2}^{2}\right)$. It can be seen that the sum of all pixels only drops down to about $70 \%$ of the aberration free brightness for severe aberrations of $2 \mathrm{rad}$. The brightness does not change noticeably till the amplitudes reaches values over $0,5 \mathrm{rad}$ from the optimum, even though the aberration can be clearly seen in the images and the STED donut will be severely distorted (see Figure 1.6). In this part of the curve deviations are visible, because the error from noise is overtaking the actual change in intensity due to the amplitude change. This means, that one image is not enough to reliably determine the brightness and many measurements have to be averaged. For the 5\%-metric it is different: the graph is steeper around the maximum and the brightness reduces to around $40 \%$ for severe aberration. The amplitude change has a bigger impact on the intensity, so the error does not effect the curve as much as before. This means, that single measurements are more reliable, also in the center region. 


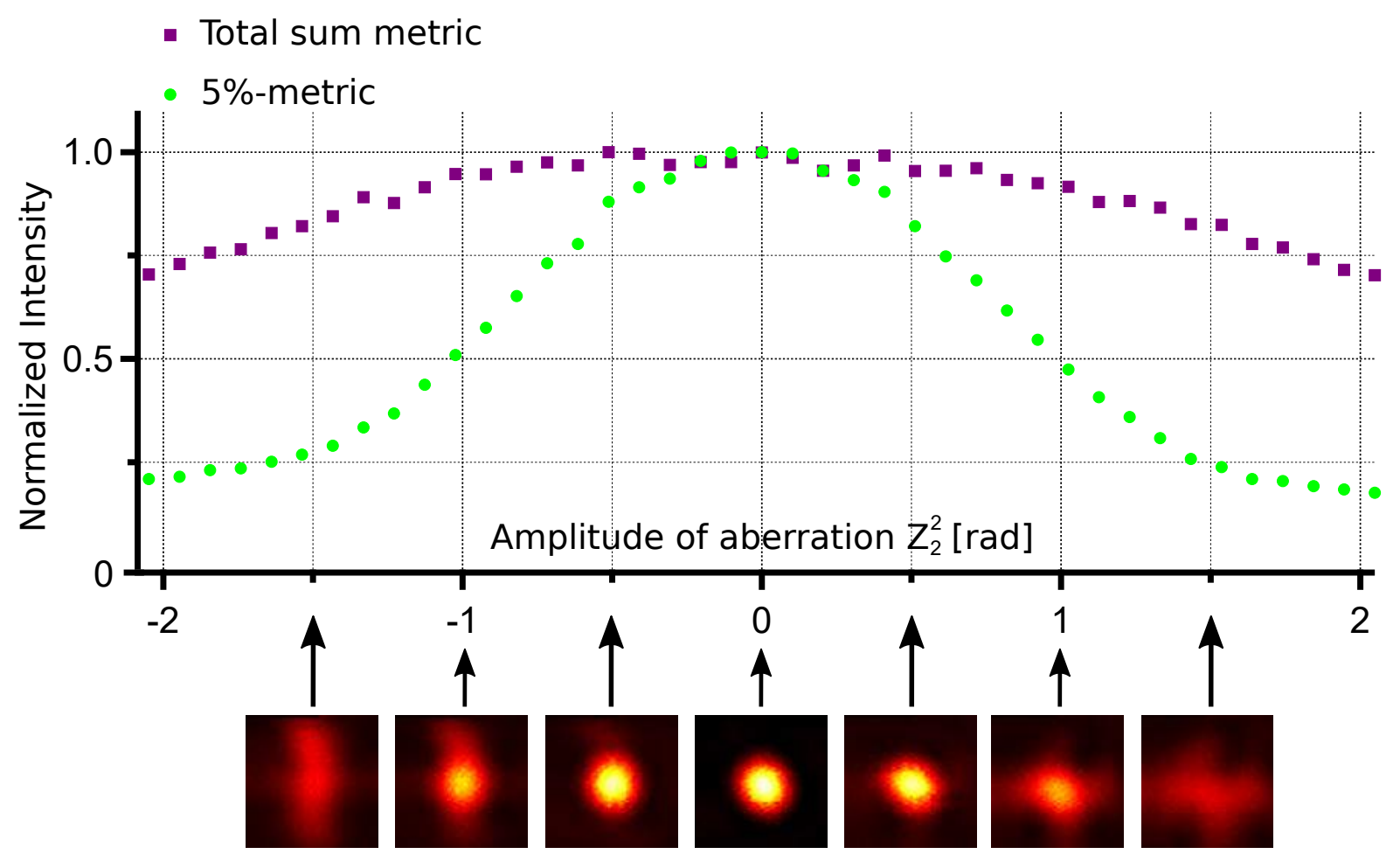

Figure 3.3: Visualization of the new metric. Images of the same gold bead are acquired with different amplitudes of astigmatism $\left(Z_{2}^{2}\right)$. The normalized value of the metric is plotted against the amplitude of the aberration. The bottom images show the beads acquired with the marked aberration amplitude. The standard metric, the total sum of all pixels, is shown with magenta boxes and the new metric, the sum of only the brightest $5 \%$ of pixels is shown with green dots.

\subsubsection{Optimization algorithm}

As described before, the goal of the new metric is to minimize the number of images and calculate the optimum amplitude for each mode. First, an aberration that is going to be corrected is chosen and the range of amplitudes is set. I typically used five amplitudes ranging from $-0.5 \mathrm{rad}$ to $0.5 \mathrm{rad}$. With each of the amplitudes an image is acquired and a metric is calculated. From the measurements the optimized amplitude of the aberration is determined. This calculated value is then set to the adaptive element and the next aberration is corrected. This procedure is repeated for all chosen Zernike modes. Since my goal is to minimize the number of images, the number of aberration modes that are corrected have to be minimized as well. It is therefore important to choose only the aberrations that will have the most impact on the image. These are spherical aberration, astigmatism and coma. Tip, tilt and defocus only position the PSF in all the three dimension and do not heavily change the shape of the focal spot. They will therefore not 
be corrected. Trefoil and the second order of aberrations are not corrected, because for my purpose reducing the number of images is of highest importance.

\subsubsection{Aberration correction}

To visualize the effect of aberrations (similar to Figure 1.6) the simulated PSFs of selected aberration modes are shown in Figure 3.4. STED PSFs, excitation PSFs as well as the effective PSFs are shown. The amplitude for each aberration is set to 0,5rad and the color scheme is normalized for each image. Since some of the effects of aberrations were already described in section 1.4.1, I mainly focus on the effective PSFs here. Astigmatism $Z_{2}^{2}$ and $\mathrm{Z}_{2}^{-2}$ only differ in the rotation of the main axis and therefore I discuss only one mode here. The same holds true for coma $\mathrm{Z}_{3}^{1}$ and $\mathrm{Z}_{3}^{-1}$ (see Table 3.1). Inhomogeneities in the intensity of the STED donut, with higher and lower intensities, results in an increased or decreased depletion respectively, resulting in non-symmetrical effective PSFs for astigmatism and coma. These two are also the aberrations with the most severe changes in the FWHM. The effective PSF for astigmatism changes from a uniform FWHM of 77nm in the unaberrated case (a) to $71 \mathrm{~nm}$ and $133 \mathrm{~nm}$ respectively in the aberrated case (b). Apart from this change in resolution the intensity drops by nearly $70 \%$ which happens because the intensity zero is filled with depletion intensity (see section 1.4.1). This is a dramatic loss of intensity in the effective PSF, even though the zero is only filled by approximately $1 \%$ of the maximum intensity. The reason for this is that the stimulated emission is non-linear and has a big effect, even for lower intensities (Figure 1.3). For coma (Figure 3.4c) the difference in the resolution is not as severe and it increases to $86 \mathrm{~nm}$ in $\mathrm{X}$ - and $95 \mathrm{~nm}$ in Y-direction. The intensity drop by $25 \%$ is mostly explained by the drop of intensity by the excitation beam alone and is not just an effect of the aberrated STED beam (Figure 1.6). The spherical aberration (d) only elongates the focus without disturbing the symmetry in lateral direction. Due to the elongation, the excitation as well as the STED beam loose intensity in the focal plane, which leads to a loss of intensity and a larger FWHM in the effective image. The effect of the aberrations on the effective PSF shows, that these aberrations need to be corrected. Since the aberrations are independent from each other and are corrected one after the other, the sequence should not play a role. However, the aberration which is present with the highest amplitude should be corrected first, to increase the signal to noise ratio of the other aberrations. Spherical aberration is usually the most dominant aberration, because of the refractive index mismatch that is always severe deep in a sample and will therefore be corrected first. For the other aberrations there is no clear difference, but astigmatism was chosen to be corrected before coma. After the sequence of aberrations is now set, the number of amplitudes which are applied need to be determined as well as the values of amplitudes and the fitting procedure. I want to apply small values from -0,5rad to 0,5rad to test the aberration, because they are usually not larger. Furthermore, it was shown that using these amplitudes lead to a more precise result (research was done in two photon excitation microscopy [91]). 


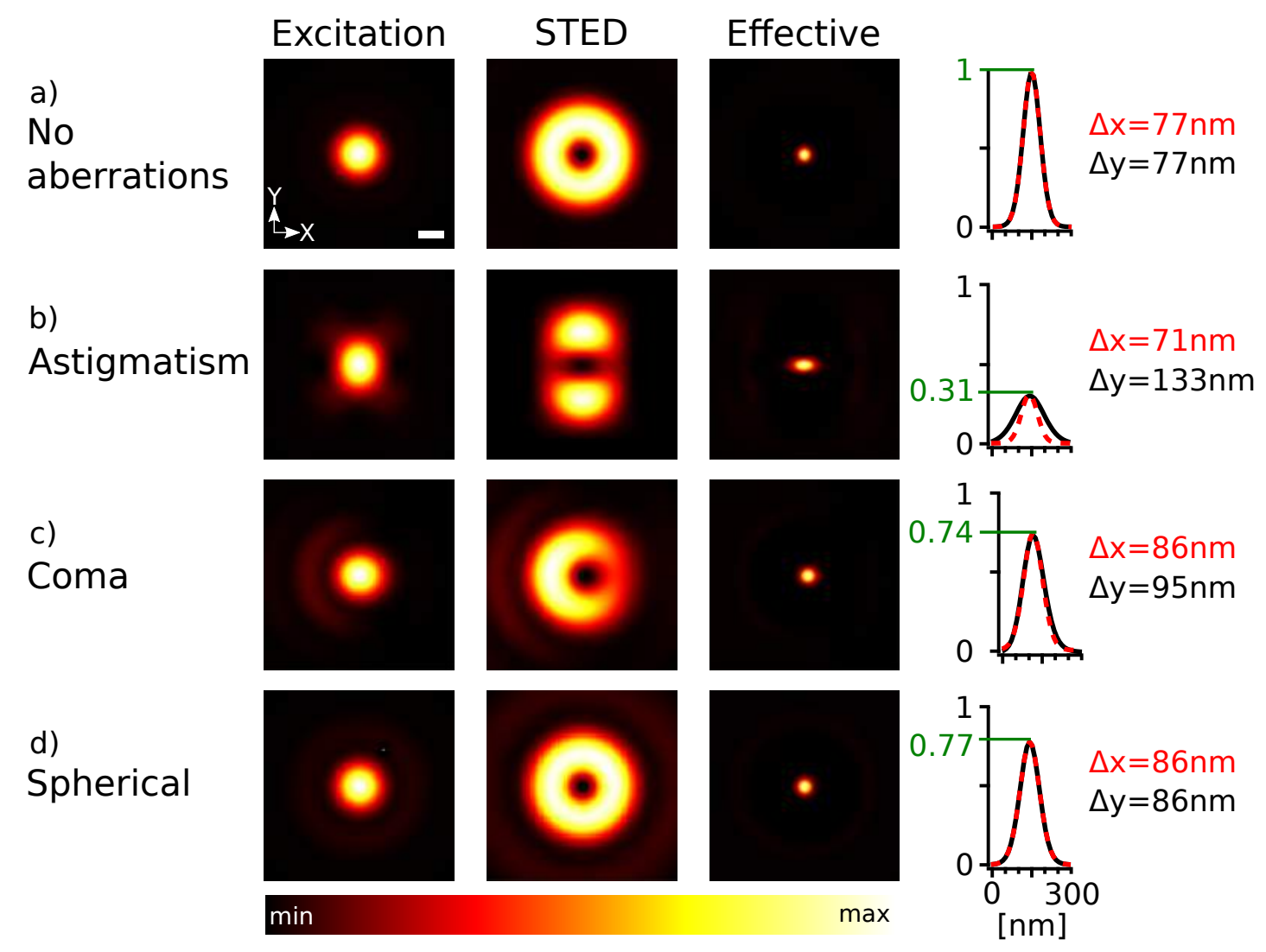

Figure 3.4: Simulated images that show the effect of selected aberrations on the PSF of the excitation beam, the STED donut and the effective PSF. Each row shows a different aberration, starting with a non-aberrated focus in a), followed by astigmatism in b) as well as coma in c) and spherical aberration in d). On the right hand side the lineprofiles in $\mathrm{X}$-(red dotted) and Y-direction (black solid) are shown. The FWHMs are given next to the plot and the maximum intensity is indicated in green. All images have the dimensions $1.5 \mu \mathrm{m} \times 1.5 \mu \mathrm{m}$.

As displayed in figure 3.3, I can approximate the central region by a parabola. The number of images taken for each aberration mode is set to be five. In principle three images would be sufficient, but the measurements were not as accurate and therefore had to be repeated. Hence, taking five images one time turned out to be more efficient than taking three images more often. To test the stability and reliability of the routine, I repeated the routine ten times for the same starting conditions. The imaging was done on $100 \mathrm{~nm}$ diameter fluorescent beads. The starting conditions were randomly set to different values for the tested aberration. The results are shown in Figure 3.5, where the standard deviation of these ten measurements are plotted against the amplitude that was found to be optimal. Here the values of three different metrics are shown, the total sum of pixel intensities, the quadratic sum of pixel intensities and the new introduced 5\%-metric. All three measurements show the same behavior, with the 5\%-metric being the most consistent overall. The standard deviation is minimal for starting amplitudes close to the 


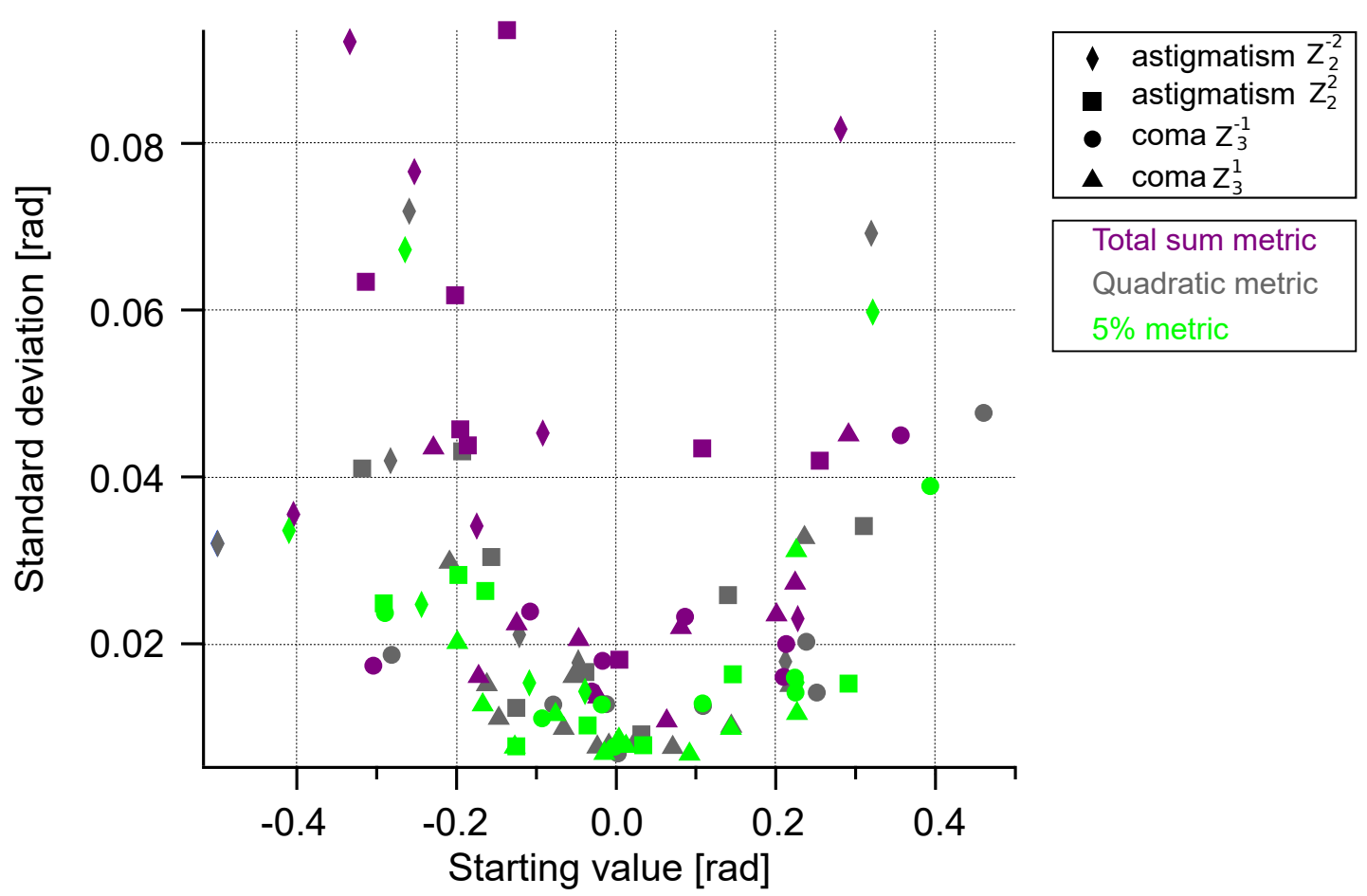

Figure 3.5: Reliability of the measurement for different metrics. Each point in the graph stands for the standard deviation of 10 runs of the routine one after the other. The $\mathrm{X}$-axis shows the random starting value. Different aberration modes have been chosen for the analysis and for each measurement five images have been recorded from $-0.5 \mathrm{rad}$ to $0.5 \mathrm{rad}$. The legend is divided into colors and shapes.

optimum. When the starting value is further away from the optimal value, the standard deviations are larger, which means, that a single experiment is not precise enough for correction. The total sum metric has the most and largest outliers and is therefore the least trustworthy metric. Quadratic and 5\%-metric are both better and when analyzing the values for starting amplitudes between -0.1rad and 0.1rad it becomes clear, that the averaged standard deviation of the 5\%-metric is 0.010rad, while the averaged standard deviation for the quadratic metric is 0.011rad. As reference, the value for the total sum metric is $0.029 \mathrm{rad}$. The highest standard deviation in the given range is 0.015 for the $5 \%$ metric and 0.021 for the quadratic metric. In the following I will use the $5 \%$-metric, since it is the most reliable and from this analysis I conclude, that I can trust a measurement if the difference between the starting amplitude and the optimized amplitude is smaller than 0.1rad. Taking this result into account I now present my algorithm to correct for aberrations, which is also visualized by a flow diagram in Figure 3.6. First, an aberration is chosen, typically spherical aberration for deep tissue imaging. For this aberration an amplitude is set at which an image is acquired and the metric is calculated. This is repeated five times with amplitude typically ranging from $-0.5 \mathrm{rad}$ to $0.5 \mathrm{rad}$. Then, the metrics are plotted against the amplitudes and by fitting a parabola to the points the optimal amplitude can be found. This amplitude is then taken as the optimized value 


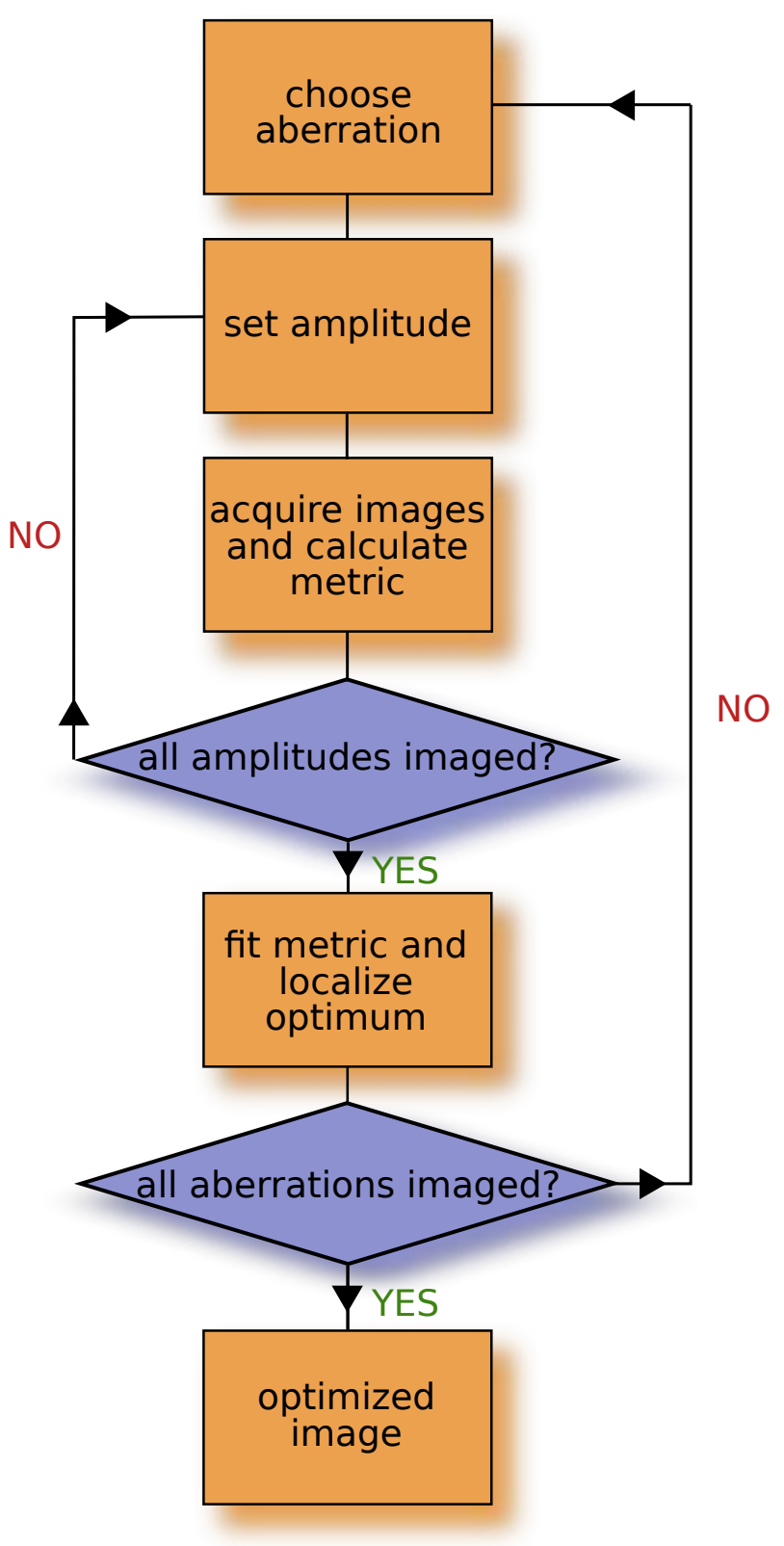

Figure 3.6: Flow diagram of the algorithm, adapted from [56]. The orange boxes describe what the routine executes in the steps and the blue boxes are switches that react to the conditions that apply.

and the next aberration mode is optimized, typically astigmatism. After both directions of astigmatism are corrected, both directions of coma will be corrected, finishing one cycle of the algorithm. If the calculated amplitude value is larger than 0.5rad or lower than -0.5rad the amplitude is set to either $0.5 \mathrm{rad}$ or $-0.5 \mathrm{rad}$. If there are optimization values that differ more than 0.1rad from the starting value, the routine is started again with all aberrations that have these large values. When all aberrations are corrected to a value where staring amplitude and optimized amplitude do not differ by more than 
$0.1 \mathrm{rad}$, the routine is stopped and the image is corrected. By doing so I have a minimum number of five images per amplitude, which makes 25 in total. In measurements I usually did not have large aberrations so two loops of the routine were enough to correct for all aberrations and a maximum of 50 images total were needed to correct for five aberrations. It is important to notice, that not only the choice of the metric is important for minimizing the number of images, but also how an accurate measurement is determined. Patton et al. [65] used repeated measurements to securely determine the optimal amplitude for the aberrations, which resulted in havening to take 50 images to correct for one amplitude. It is however enough to once optimize the amplitude on a position that is already close to the optimal amplitude. Therefore, I stop optimizing an aberration when the starting amplitude of the routine is closer than 0.1rad to the optimized amplitude.

\subsubsection{Software implementation of the optimization routine}

This section will explain how the routine is built. Two programs have been connected to be able to run the routine. The first being Imspector (Abberior Instruments) which is used to do the imaging and Casao (Imagine Optic), which is the software that controls the deformable mirror. As programming language Python (Python Software Foundation [94]) is chosen. One reason for this is, that Imspector comes with a Python Interface which is named specpy and can be run from an external console [95]. This means, that the data can be transfered and analyzed to the Python program in real time. Even Imspectors analyzing tools can be used from there. The second program used does not have a Python interface but can be controlled via the programming language $\mathrm{C}$. The Python package ctypes is used to control it with a small block of $\mathrm{C}$ code in Python. Therefore, I can take control of this program as well. Together the two can acquire the image and control the shape of the DM and both can be handled from a self written Python script. Jacopo Antonello from the group of M.Booth was so kind to provide some help and shared a version of the program they used in [56].

\subsection{Characterization of the deformable mirror}

While working with the deformable mirror I figured out, that it shows unexpected behavior when exposed to high laser powers. The mirror changes its surface which leads to additional aberrations depending on the STED laser power of the beam that is reflected. This was measured in the same way the deformable mirror was initially calibrated, by focusing onto silver mirror (section 3.2.1). The wavefront was analyzed by the WFS for different STED powers. Shown in Figure 3.7 are the amplitudes of aberration that are the the result to the DM when using a laser beam with higher powers. The amplitudes increase when the power increases and all aberration modes show linear increase with the laser power. The modes that show the strongest influence to the high power are tilt $\left(Z_{1}^{1}\right)$, defocus $\left(Z_{2}^{0}\right)$ and astigmatism at $0^{\circ}\left(Z_{2}^{-2}\right)$. The change in coma and spherical aberrations 


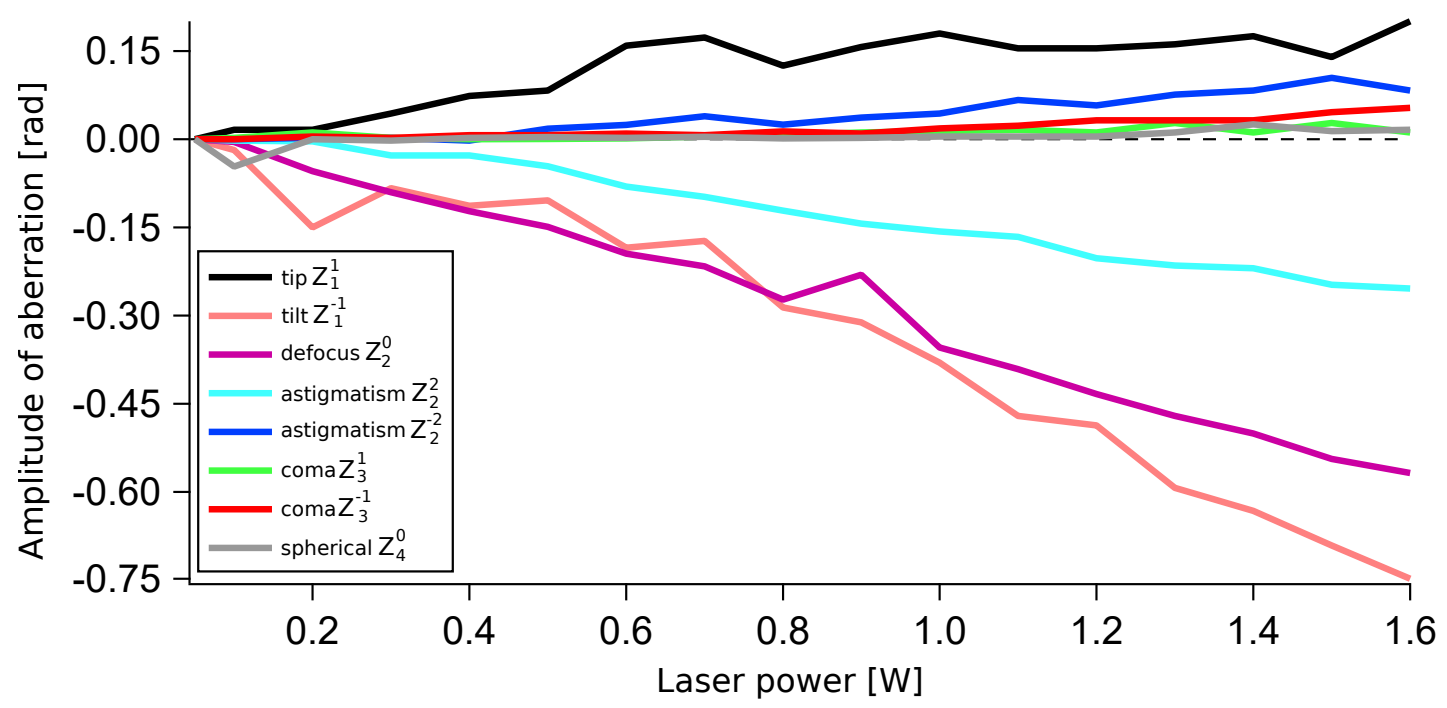

Figure 3.7: Influence of the STED power on the deformable mirror. The data has been recorded with the WFS. The power of the laser was increased in increments of $0.1 \mathrm{~W}$.

is negligible. In Figure 3.8 the resulting STED donuts and the surface change on the deformable mirror are shown. At low power, the donut is round and the wavefront is flat, since this is the reference setting. Applying medium STED power $(0,5 \mathrm{~W}$ output power, resulting in $180 \mathrm{~mW}$ power on the mirror) results in a donut that shows astigmatism. Tip, tilt and defocus cannot be seen in this image since the focal plane was adjusted and the donut was centered in the frame. Tilt and defocus can however be seen in the shape of the deformable mirror, resulting in a curved surface that is tilted to the top. With the high power laser beam $(1,6 \mathrm{~W}$ output power, $490 \mathrm{~mW}$ on the $\mathrm{DM})$, the astigmatism leaves two separated spots instead of the donut shape. The shape of the mirror shows more severe defocus and tilt which results in a valley that is open to one side. An explanation for this behavior cannot be given and also the company Imagine Optic did not know about this before. A possible reason might be a thermal effect, because the change takes up to two seconds to appear and they have no other explanation. Regardless of this, I used the mirror because the effect was constant over weeks and when using higher laser powers a correction was applied to the DM to counteract these changes. This does decrease imaging speed however because a small routine has to be run between the imaging of confocal and STED images. 


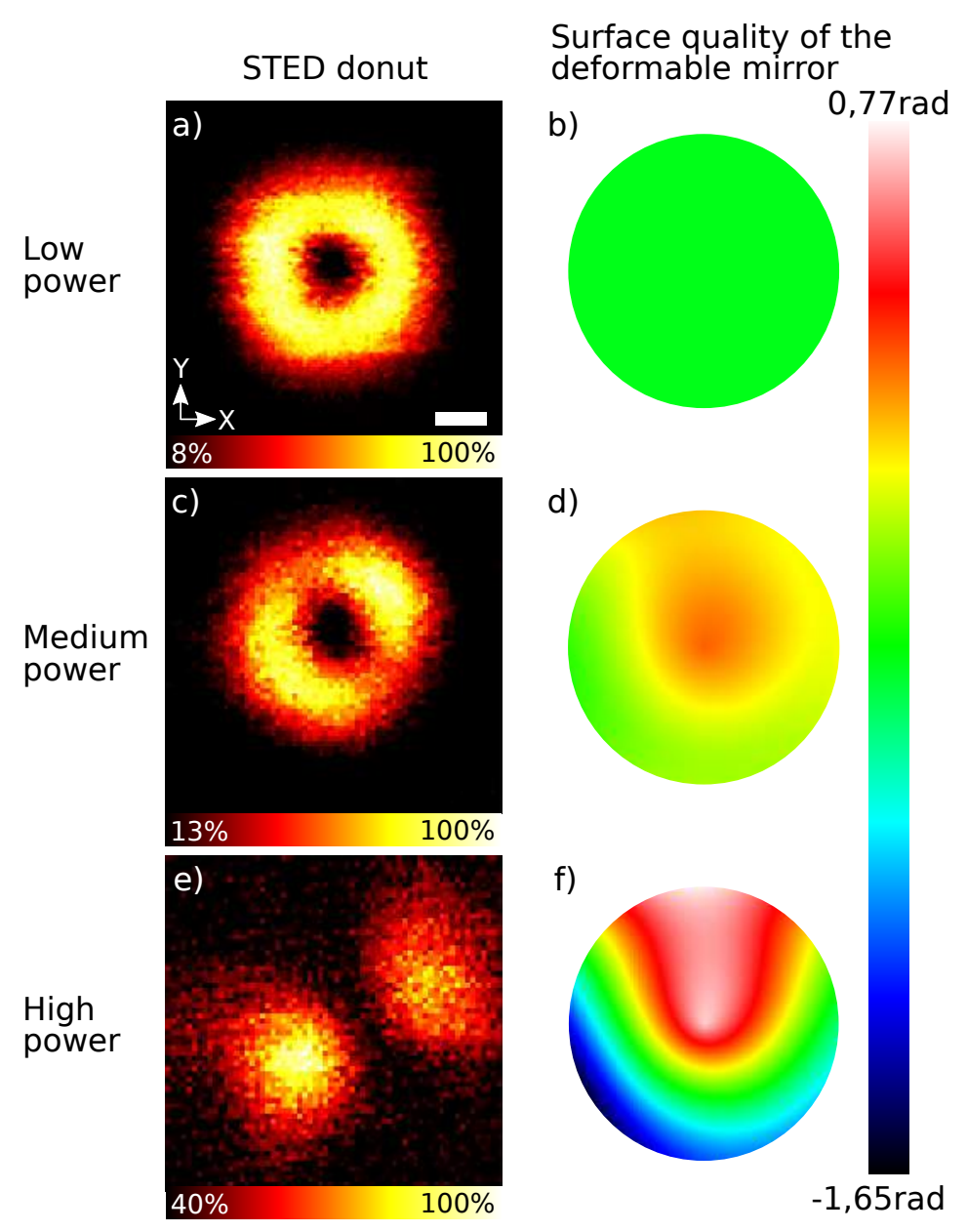

Figure 3.8: Visualization of the DM surface changes and STED PSFs at high STED laser intensities. Images a), c) and e) show the STED PSF imaged with low (1mW output power), medium $(0,5 \mathrm{~W})$ and high power $(1,6 \mathrm{~W}) . \mathrm{b}), \mathrm{d})$ and $\mathrm{f}$ ) show the respective shape of the deformable mirror. The PSF was always centered and refocused, so tip, tilt and defocus cannot be seen. The astigmatism is clearly visible in c) and e). In d) and f) the strong defocus and tilt are dominating the shape of the mirror.

\subsection{Correction of brain slice induced aberrations}

To test the optimization routine a small, photo stable fluorescent probe is needed for which I used 100nm and 40nm fluorescent beads (Crimson beads, Invitrogen, Carlsbad, CA, USA). I used slices of fixed mouse brain to create an aberrating sample. I immobilized the fluorescent beads on a microscope slide and placed a thin slice of varying thickness of brain tissue (layer one projections of cortex) on top. Another coverslip with attached beads is placed on top to create a sandwich structure of beads with the brain tissue in between. The tissue was embedded with Mowiol, and sealed with two component glue to fix it to the glass slide (Figure 3.9). Using the sandwich structure makes it possible to first correct the beads close to the surface as a reference. The settings of the reference image 
are used as a starting point for the algorithm when optimizing the aberrations deeper in the sample. Aberrations induced by the brain slice can therefore be measured in reference to the surface. Furthermore it is possible to measure the thickness of the brain slice.

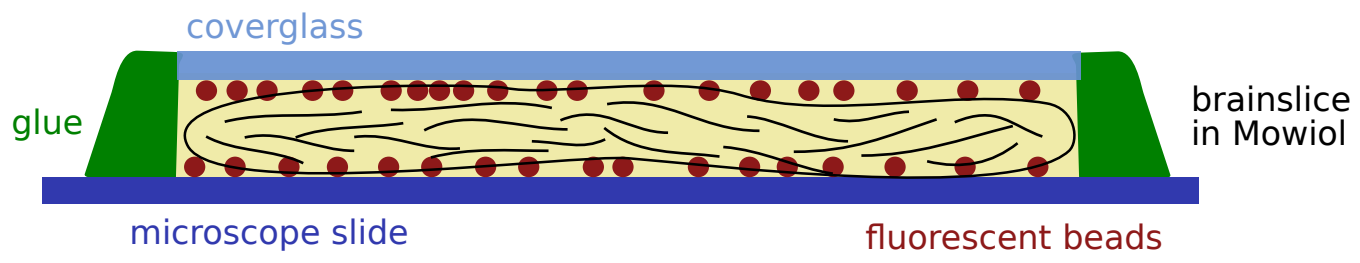

Figure 3.9: Sketch of the test sample. A brain slice is placed between two layers of fluorescent beads. It is embedded in Mowiol and sealed with glue.

For the samples with 100nm beads I used brainslices with a thickness of $20 \mu \mathrm{m}, 45 \mu \mathrm{m}$ and $75 \mu \mathrm{m}$. It is worth noting, that the thickness is measured directly in the sample and on different locations. The values varied more for the larger samples and the given values are averages. The slices were cut using a vibrotome settings of $40 \mu \mathrm{m}, 80 \mu \mathrm{m}$ and $120 \mu \mathrm{m}$. After focusing on the layer of fluorescent beads underneath the brain slice, the Z-position of the stage is noted. A fluorescent overview is recorded, before one single bead is chosen and the routine is executed. Therefore, a sequence of $1 \mu \mathrm{m} \times 1 \mu \mathrm{m}$ images are taken, as explained before. After the correction the bead is imaged with the STED donut applied at a power of $360 \mathrm{~mW}$ STED power in the back aperture of the objective. As an example, confocal and STED images with and without correction at a depth of $46 \mu \mathrm{m}$ are shown in Figure 3.10. The images were acquired with a pixel size of $20 \mathrm{~nm}$ and $0.5 \mathrm{~ms}$ pixel dwell time. The laser powers were $\sim 20 \mu \mathrm{W}$ for excitation and $360 \mathrm{~mW}$ for STED. The confocal images in Figure 3.10a) show the uncorrected and correct images of a 100nm bead in $\mathrm{XY}$ - and XZ-scans. The peak brightness in the corrected image is nearly doubled from 78 counts to 149 counts in the XY-image. Furthermore, in the XZ-scan it can be clearly seen, that the focal length in the corrected image is significantly shorter. This observation is confirmed by the $\sim 2$ fold decrease in FWHM in FWHM of the lineprofiles (average over three pixels, Figure 3.10 b). While the XY-size decreases slightly, the decrease in focal length in the Z-direction is very significant. This also explains the increased brightness, since the light is focused into a smaller volume. The STED images shown in Figure 3.10 ) are of the same bead that was imaged in confocal mode and are taken with the same imaging parameters. The peak brightness in the corrected STED image increases by $20 \%$ and even more important, the bead is smaller in size and looks less blurry. This can be confirmed in Figure 3.10d), where the FWHM in X-direction of the corrected image reduces to $64 \%$ of its original size. For the Z-direction the size is reduced to around two thirds. The FWHM in Z-direction should be the same for confocal and STED and do not differ much in this measurement. The averaged results of all three different thicknesses can be seen in Table 3.2. The data was acquired by correcting the aberrations of a bead near the center region of a $10 \mu \mathrm{m} \times 10 \mu \mathrm{m}$ image and then taking STED images of the region 
a) Confocal

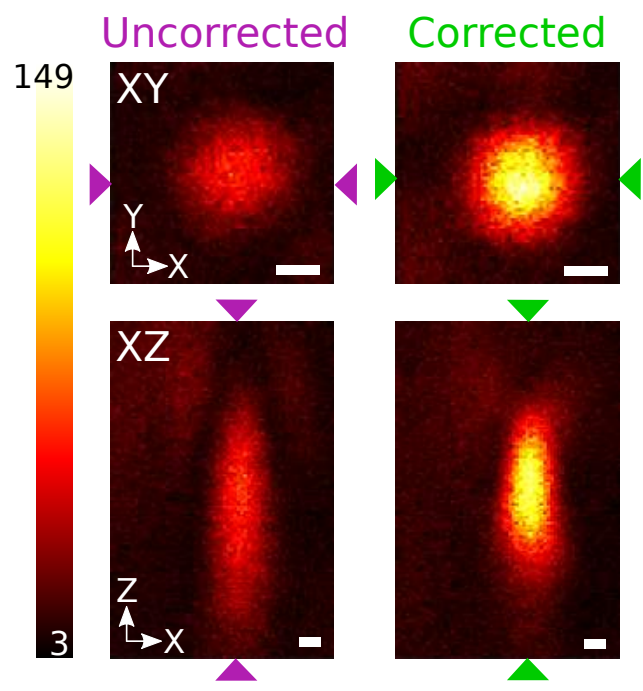

c) STED

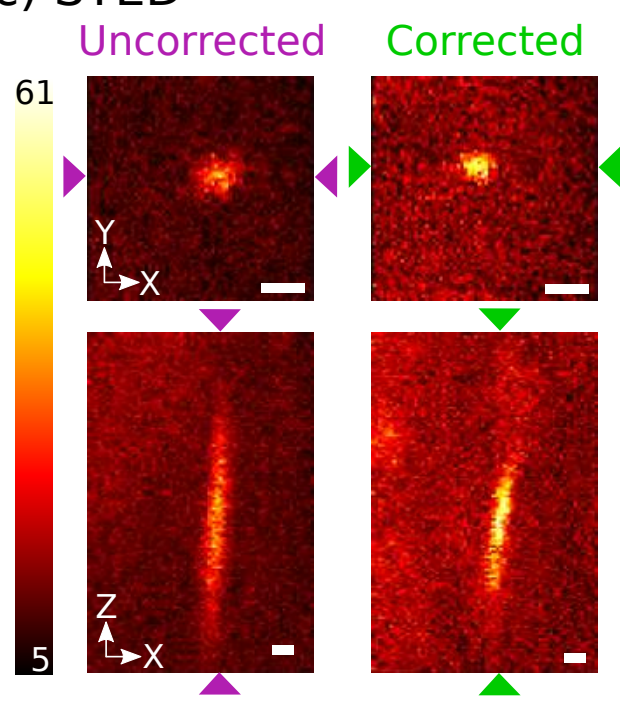

b)

$$
\Delta \mathrm{x}=329 \mathrm{~nm}
$$$$
\Delta \mathrm{x}=359 \mathrm{~nm}
$$
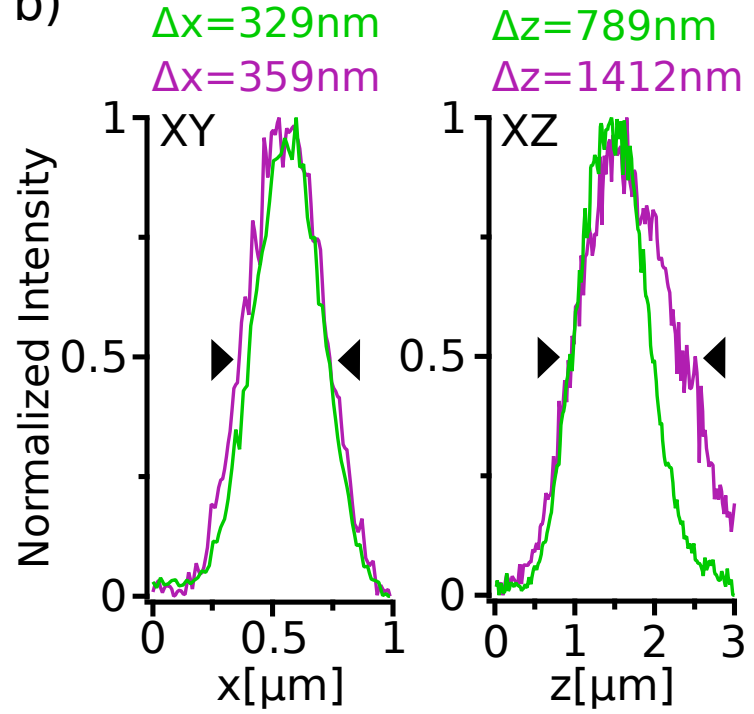

d)
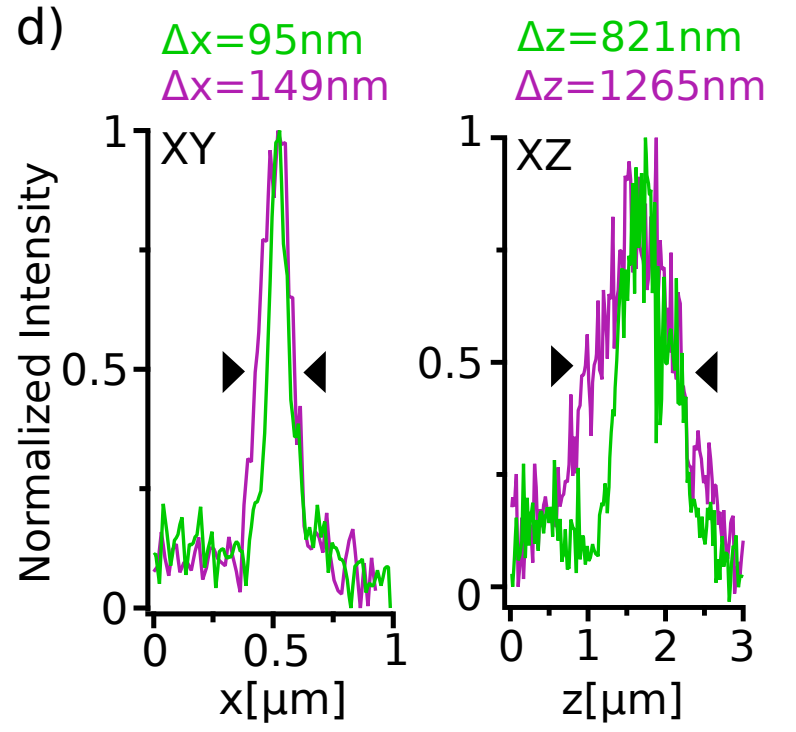

Figure 3.10: 100nm fluorescent bead underneath $45 \mu \mathrm{m}$ of mouse brain. a) uncorrected and corrected image of one bead imaged in confocal mode (all raw data). The XY-Scan is shown above the XZ-scan. b) lineprofile (width of three pixels) at the positions indicated by the green and magenta arrows. The FWHM of a Gaussian fit are printed. Accordingly c) STED images of the bead in XYand XZ-direction, each accordingly with and without the correction applied. Again, the lineprofiles as well as the according FWHM are shown. For the X-direction a Lorentzian function was fitted, instead of a Gaussian. Scalebar in the images: $200 \mathrm{~nm}$.

with the correction and without. The data was then analyzed by drawing a lineprofile of the width of three pixels in X-direction, over single beads. The beads were chosen by me, carefully selecting the smallest structures with a well enough signal to noise ratio. The FWHM was then measured in the Imspector software. As an example, an overview is 


\begin{tabular}{l|ccc} 
& \multicolumn{3}{|c}{ Thickness of the brain slice } \\
& $20 \mu \mathrm{m}$ & $45 \mu \mathrm{m}$ & $75 \mu \mathrm{m}$ \\
\hline FWHM uncorrected $[\mathrm{nm}]$ & $108 \pm 9$ & $143 \pm 22$ & $165 \pm 16$ \\
FWHM corrected $[\mathrm{nm}]$ & $98 \pm 5$ & $108 \pm 16$ & $119 \pm 10$
\end{tabular}

Table 3.2: FWHM in corrected and uncorrected STED images of 100nm fluorescent beads underneath brain slices of varying thickness. Imaged with a STED power of $360 \mathrm{~mW}$ in the back aperture of the objective.
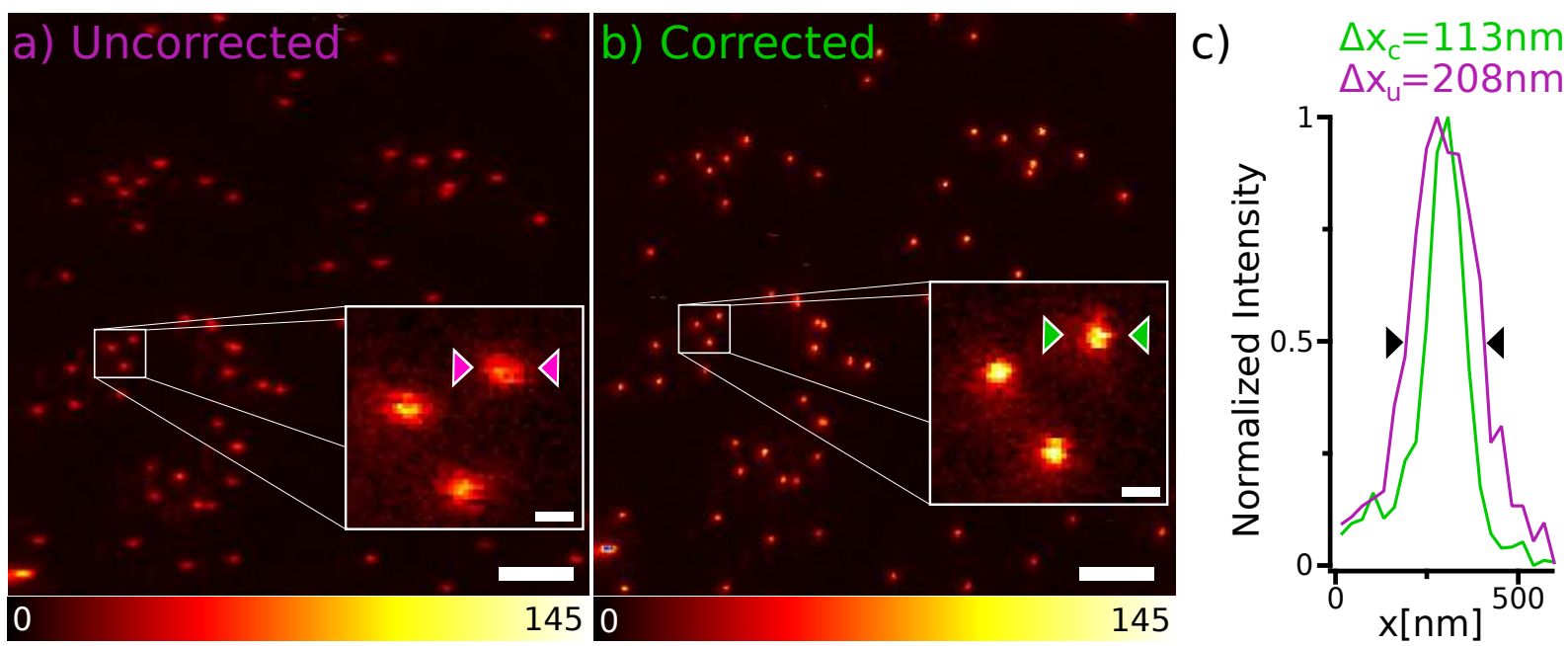

Figure 3.11: STED images of 100nm fluorescent beads underneath $79 \mu \mathrm{m}$ of brain tissue. a) uncorrected STED image of fluorescent beads with inset (raw data). In b) the correction was applied. c) lineprofiles (width of three pixels) at the locations indicated by the magenta and green arrows. The FWHM was calculated by a Lorentzian fit. Scalebars: $1 \mu \mathrm{m}$, Scalebar inset: 200nm.

shown in Figure 3.11. The images were acquired with a pixel size of $30 \mathrm{~nm}$ and $0.5 \mathrm{~ms}$ pixel dwell time. The laser powers were $\sim 23 \mu \mathrm{W}$ for excitation and $360 \mathrm{~mW}$ for STED. This was done on $100 \mathrm{~nm}$ beads underneath $79 \mu \mathrm{m}$ of brain tissue. The same area is scanned twice, once with the correction and once without. Both are STED images with an inset to enhance three of the beads. The positions of the lineprofiles are marked in magenta and green and the data is shown on the right side. In this image the FWHM in X-direction was improved from $208 \mathrm{~nm}$ to $113 \mathrm{~nm}$. Furthermore the improvement in brightness can be seen in the images. To test the performance of the STED microscope I used the correction also on smaller beads of 40nm diameter. These beads have weaker fluorescence and bleach faster and are therefore more difficult to correct aberrations on. The preparation of the samples was similar to before with the change that $40 \mathrm{~nm}$ beads instead of $100 \mathrm{~nm}$ beads are used. The beads were imaged with a STED power of $144 \mathrm{~mW}$ in the back aperture of the objective. The improvement of the correction can be seen in Table 3.3 . The 


\begin{tabular}{l|ccc} 
& \multicolumn{3}{|c}{ Thickness of the brain slice } \\
& $20 \mu \mathrm{m}$ & $30 \mu \mathrm{m}$ & $45 \mu \mathrm{m}$ \\
\hline FWHM uncorrected $[\mathrm{nm}]$ & $98 \pm 6$ & $119 \pm 9$ & $129 \pm 17$ \\
FWHM corrected $[\mathrm{nm}]$ & $71 \pm 13$ & $79 \pm 6$ & $85 \pm 13$
\end{tabular}

Table 3.3: FWHM in corrected and uncorrected STED images of 40nm fluorescent beads underneath brain slices of varying thickness. Imaged with a STED power of $144 \mathrm{~mW}$ in the back aperture of the objective.

FWHM under $45 \mu \mathrm{m}$ of tissue can be decreased from $129 \mathrm{~nm}$ to $85 \mathrm{~nm}$. Typically 5 to 10 beads in the same $10 \mu \mathrm{m} \times 10 \mu \mathrm{m}$ area were used to correct for aberrations. To analyze if the aberrations are only depending on the thickness of the sample, the aberrations were averaged for each area. The averages do vary from area to area and also the standard deviations differ. This is shown for four areas on the same $47 \mu \mathrm{m}$ thick sample in Figure 3.12 .

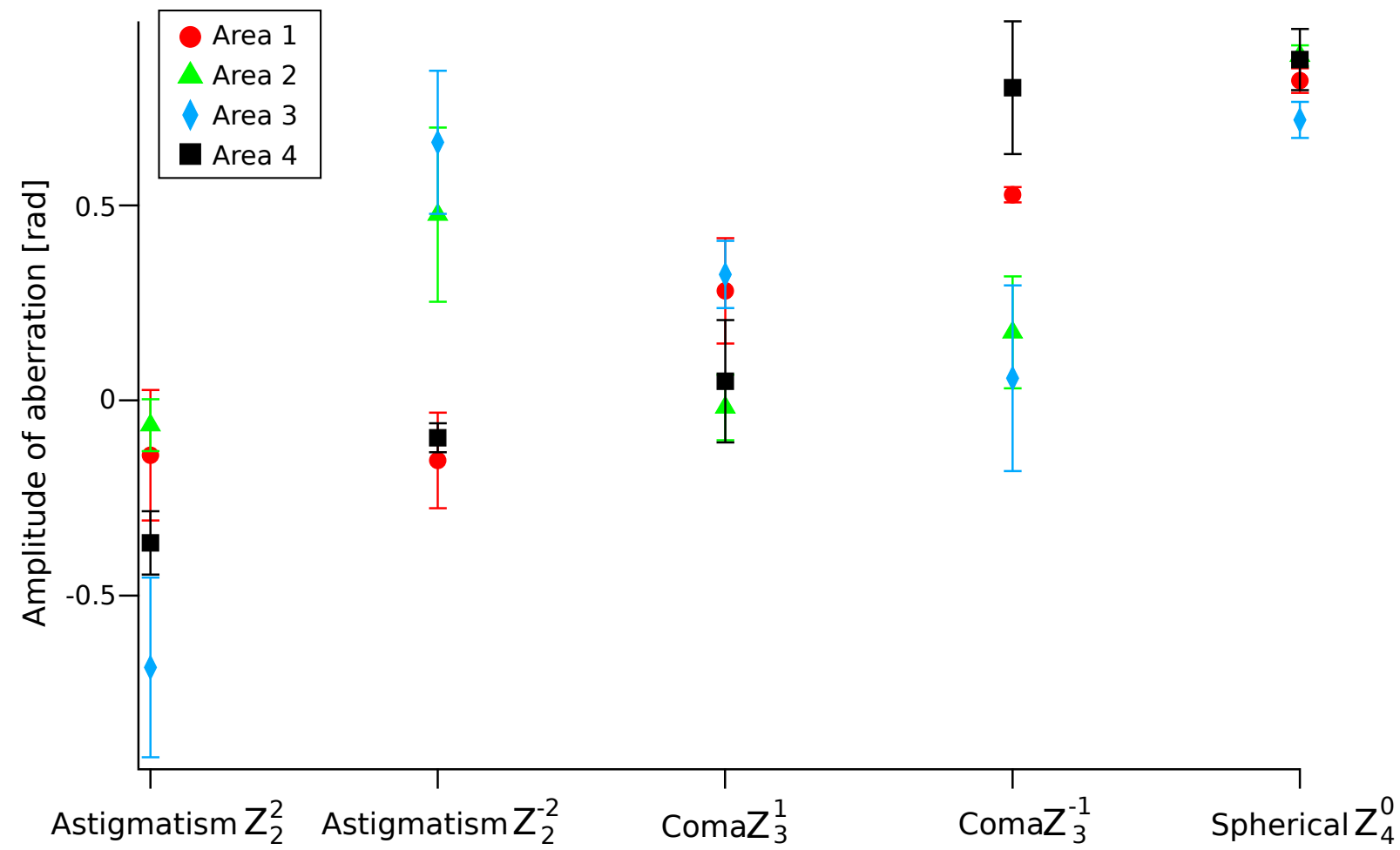

Figure 3.12: Amplitudes of aberration in different areas underneath $47 \mu \mathrm{m}$ of mouse brain tissue.

The average values are plotted in differing colors and shapes and the standard deviation is indicated. Taking $Z_{2}^{-2}$ as example, the averages of area 1 and area 3 differ from $-0.15 \pm 0.12 \mathrm{rad}$ to $0.66 \pm 0.18 \mathrm{rad}$. In the same areas the amplitudes of $Z_{1}^{3}$ only differs from $0.33 \pm 0.08 \mathrm{rad}$ to $0.28 \pm 0.14 \mathrm{rad}$ and can therefore be considered the same. It is also worth 
noting, that the errors are vastly different. That means, there might be a difference in aberrations on beads that are just micrometers apart from each other or the aberrations can be constant over a larger field of view. The significant differences in the behavior of astigmatism and coma between the different areas shows, that there can be no general aberration for a specific depth, since the aberrations depend more on the region than on the depth and the amplitudes of aberrations are independent from each other. The only exception from this is spherical aberration, that shows fairly consistent aberration values in different areas. Spherical aberrations are known to be dependent on the depth, as it is already described in the literature [96]. I also observed this consistently during the measurements (data not shown). Since the spherical aberrations depend strongly on the depth in which the image is taken, it is possible to estimate the spherical aberrations beforehand. This reduces the optimization of the spherical aberrations to one cycle of five images and also makes it easier to find the beads in the first place. 


\section{Discussion}

\subsection{D and two-color STED microscopy}

My first goal was to build a 3D two-color STED microscope attached to an inverted microscope stand, (for easy sample alignment) to do co-localization studies and 3D resolution imaging. This goal was achieved with the developed setup in which even live cell imaging can be performed (section 2.3). By using just one STED beam, the co-localization in the STED image is a build-in feature and scanning each line twice, alternating between excitation I and II as well as detection I and II, secures minimal crosstalk of the channels. However, doubling the number of lines does have the drawback of being slower than one color scanning. Göttfert et al. [97] showed, that it is also possible to acquire a two-color image by using interleaved pulses, hence only scanning once. To realize this a depletion laser of $20 \mathrm{MHz}$ was used and the excitation lasers were pulsed at $10 \mathrm{MHz}$, with a time frame of 100ns between them. Every 50ns there will be an excitation pulse, followed by a STED pulse, but excitations I and II alternate. This can be observed by two detection channels that use a gating mechanism to only record signal during certain time periods.

The field of view of my microscope is limited to an $80 \mu \mathrm{m} \times 80 \mu \mathrm{m}$ area. I use a simple scanner, consisting of glavanometric mirrors. The mirrors are placed close together and the conjugated plane of the objective has to be placed between them. This reduces the aberrations and misalignment of excitation and STED beam when the scanning area is too far of center [98], but will not eliminate them. A way of avoiding aberrations is to use four mirrors in total, which is realized in a quadscanner (Abberior Instruments, used in [99]).

Instead of using a phaseplate to create the typical donut shape, an SLM is used. This adds more versatility in the setup by making it possible to correct for aberrations. I mostly corrected aberrations of the system, but correcting specimen induced aberrations is also possible. Also, with the SLM it is possible to create a 3D STED PSF by applying a PPP phase pattern. If one would use a phase plate made of glass, the VPP and PPP phase pattern would be created on different beams that need to be recombined to form the final STED PSF [21]. Since the creation of both phase patterns is done on the same beam with the SLM, a beam recombination is not necessary. Furthermore, the intensity of both beam patterns can be adjusted easily by adjusting the polarization of the beam electronically, using a HWP in front of the SLM. Using the SLM for PPP and VPP allows to image fluorescent structures with superresolution in all three dimensions. The FWHM of the effective PSF during the 3D measurement was optimized to $76 \mathrm{~nm} \times 76 \mathrm{~nm} \times 145 \mathrm{~nm}$. 
It would be best to image with an isotropical resolution in all three dimensions, but when doing so, I only reach a FWHM of $120 \mathrm{~nm}$ in each direction. I chose to sacrifice some resolution in the axial direction for better resolution in the lateral direction.

The FWHM of the two imaged fluorophores Alexa Fluor 594 and STAR RED are $54 \mathrm{~nm}$ and $39 \mathrm{~nm}$ in 2D STED microscopy. This difference can be mainly explained by the different stimulated emission cross sections of the two dyes at $775 \mathrm{~nm}$. The stimulated emission wavelength is much closer to the emission maximum of STAR RED than of Alexa Fluor 594 and therefore the depletion is more efficient in STAR RED. Hence, the two dyes show a difference in FWHM.

\subsection{Adaptive optics}

I showed, that aberration correction is possible in STED microscopy with an approach, that uses only one adaptive element (a deformable mirror) for all beams and by correcting only five aberration modes (section 3). For STED microscopy two laser beams of different wavelength are required. The excitation at $650 \mathrm{~nm}$ differs by $125 \mathrm{~nm}$ from the STED wavelength at $775 \mathrm{~nm}$. With only one deformable mirror I cannot account for both wavelength at the same time. I therefore assumed, that the difference in OPL can be neglected between excitation and STED beam. This means, that while I was adjusting the aberrations with the excitation wavelength, aberrations might still be present in the STED beam, which might decreases the imaging capabilities. The magnitude of aberration in the STED beam is hard to quantify as it cannot be measured easily. To correct the aberrations of excitation and STED beam individually, an additional adaptive element needs to be placed in the STED beam path. This has been shown to be efficient in the work by Patton et al. which used an additional SLM for the STED beam. This was especially important for 3D STED microscopy [65]. However, this results in an even more advanced setup with many more degrees of freedom which need to be adjusted. Since the aberrations in the STED beam need to be corrected additionally, the number of images that have to be taken for correction increases even more.

\subsubsection{Correcting the PSF movements from aberration modes}

A problem for the correction is that the aberrations are not always independent of each other. This means, that if I apply e.g. coma to the deformable mirror the position of the focal spot will be shifted slightly. It is important to keep the PSF centered in the focal plane for all amplitudes of aberrations to have comparable brightness. To avoid movement of the focus during the correction the shift has been eliminated by adding tip, tilt and defocus. Most critical was the correction of spherical aberration with defocus, since the change of focal plane is an essential part of the aberration. It is challenging to estimate the focal plane from a stretched out focus, because it is lacking a well defined peak in the axial direction. The amplitude of spherical aberrations is mostly dependent 
on the depth and can therefore be estimated roughly. Also, since spherical aberrations are nearly constant in a sample at a certain depth, the amplitude can be measured first and on nearly any structure thereby having a good estimate for the whole sample (Figure 3.12 .

\subsubsection{The adaptive optics setup}

Unfortunately the deformable mirror that was used in this setup showed unexpected behavior when exposed to high laser powers. As presented, unwanted aberrations are introduced to the beam, that are roughly proportional to the applied laser power. I was only able to work with the deformable mirror because the power dependency was reproducible. I measured the aberrations for different laser powers and corrected for them by applying the inverted aberrations to the mirror. Still, it leaves a source of uncertainty if a device is not working as it is supposed to. As these deformations appeared at high STED laser powers, I had to apply a different set of corrections when switching between confocal and STED mode and the correction depends on the power of the STED beam. This set of corrections had to be measured at least weekly for each STED power. The different deformations at high and low STED power also made it challenging to overlay the excitation and STED beam, since the high power STED beam and excitation beam cannot be observed simultaneously. To observe the high power STED beam on the PMT, gray filters are used to reduce the intensity of the light. Therefore the far less intense excitation light cannot be seen on the PMT anymore. This leaves room for improvement in the adjustment of the microscope, since I adjusted and overlaid the low power beams and could not optimize after the correction of the high power effect.

Using a scanning stage in the setup has the advantage that the beam path is kept simple. However, a disadvantage is a smaller field of view (maximum $20 \mu \mathrm{m} \times 20 \mu \mathrm{m}$ ) and a slower scanning speed. The slow scanning is the most time consuming part of my correction routine, which makes the runtime of the calculations and setting time of the deformable mirror negligible.

\subsubsection{The optimization routine}

Patton et al. have shown that aberration correction is possible, even in 3D STED microscopy [65]. However, they used a complicated setup with a deformable mirror for all beams and an additional SLM just for the STED beam. Furthermore, they need to take at least 50 images per aberration mode (sometimes 150 per mode), just to do the correction of the deformable mirror. Afterwards they need to take even more images to correct for additional aberrations in the STED beam. The 3D STED PSF is even more susceptible to aberrations than the 2D STED PSF, which is why the STED beam has to be corrected precisely. I set out to built a simpler setup using just one deformable mirror to correct for the largest aberrations and using as few images as possible. With photobleaching in the 
sample the number of images needs to be minimal. The optimization process I developed uses the 5\%-metric presented in this thesis. I showed that the routine leads to reliable results for just 5 to 10 images per aberration mode (section 3.3.4). I have tested my routine with fluorescent beads beneath brain slices of varying thickness (section 3.5) and shown improvements in all of them (section 3.5). The reason why Patton et al. take 50 images per aberration mode is, that they average over ten images per mode and amplitude. I elimiated the need to average by showing that the algorithm finds the optimal amplitude reliably. The reliability improves when the starting amplitude is close to the optimal amplitude (Figure 3.5). This means, that one loop of the routine is enough to reliably correct small aberrations. If the routine measures a large amplitude, the measured value gets applied to the deformable mirror and the routine is started again. This step by step adjustment did not need more than a maximum of three steps during the experiments and usually two loops (10 images) were enough to correct the aberration reliably.

In principle this is not limited to only one metric. I compared three metrics here, where the total sum of pixels was not as reliable as the quadratic metric and the 5\%metric (section 3.3.4).

My correction was done with five aberration modes and used a maximum of 45 images ( 5 images for spherical, $2 \times 10$ for astigmatism and $2 \times 10$ for coma). Bleaching had no visible effects on the fluorescent beads that were tested. Even if bleaching is stronger, there must be a noticeable effect within five consecutive images to impact the optimization, as only these images are compared to each other. A bleaching correction can be implemented by retaking an image with the settings of the first image and calculating the differences between the first and sixths image. Assuming a linear bleaching effect, it can be corrected requiring only one additional image per aberration mode by just adding one more image per aberration mode.

\subsubsection{Correction of brain slice induced aberrations}

To test the routine with samples that resemble the aberrations in the brain, I used fluorescent beads of $100 \mathrm{~nm}$ diameter and placed them underneath brain slices with varying thickness (section 3.5). Even if the perfect optimization is found on a small area (typically an area of $1 \mu \mathrm{m} \times 1 \mu \mathrm{m}$ was used for optimization), it does not mean that an aberration free image can be taken of a larger field of view. In Figure 3.12 I have shown that the optimizations found in different areas of the same sample differ largely. The Figure shows the averaged values of the amplitudes of 5 to 10 beads in an area of $10 \mu \mathrm{m} \times 10 \mu \mathrm{m}$. The amplitudes differ, even though they are taken in the same sample at approximately the same depth. Hence, the aberrations need to be corrected for each region individually. In some areas the aberration changes even within $10 \mu \mathrm{m} \times 10 \mu \mathrm{m}$, so the aberrations are not constant over a larger field of view. Therefore, it is crucial for the best possible correction to correct directly on the location that is of interest. The drawback of this is, that photobleaching will reduce the maximum intensity in the final image, therefore as few 
images as possible should be taken in the optimization routine. For an approximation the aberrations of a spot close to the area of interest can be taken for aberration correction. Another aspect is, that I did not correct for all aberrations so there are still higher order aberrations present. In locations where these aberrations are large the correction from the algorithm will not be enough to achieved the expected resolution. This is a possible reason why the corrected FWHM for thicker samples are larger than for thin samples (Tables 3.2 and 3.3). The 100nm beads underneath $20 \mu \mathrm{m}$ of brain tissue were corrected to a FWHM of $98 \pm 5 \mathrm{~nm}$, while the beads underneath $75 \mu \mathrm{m}$ of brain tissue could only be measured with $119 \pm 10 \mathrm{~nm}$ FWHM. In fact, it might be worth to include trefoil into the correction, as Li et al. [100] showed in simulations, that it has a strong effect on the STED images. In this publication they also show, that apart from astigmatism, amplitudes of aberrations of 0.1rad or lower do not effect the effective image largely. I also observed this but did not quantify the effect. To reduce the number of images further, the parameters of the individual images can also be adjusted to decrease photobleaching and lower acquisition time. For the total intensity metric it was shown that the number of pixels does not influence the result of the optimization, so the number of pixels per image can be optimized [88]. Furthermore the dwelltime of the pixels can be further reduced to speed up imaging and lower photobleaching.

\subsubsection{Adaptive optics in in vivo measurements}

The goal of my thesis was to show that aberration correction is possible with a simple setup and a low number of images. This was motivated by in vivo experiments in the living mouse where the penetration capability needs to be optimized for deep tissue imaging [101. Even though I showed that it is possible to correct for aberrations underneath brain tissue in a depth of up to $\sim 80 \mu \mathrm{m}$, correcting aberrations in vivo will be more challenging. One reason is that antibodies cannot be used inside the mouse brain and therefore labeling relys on fluorescent proteins. These are darker than antibodies, which are typically carrying an organic fluorophore and are also more susceptible to bleaching. Imaging and optimizing with on 40nm fluorescent beads has already been more challenging than on 100nm beads, as they emit weaker fluorescence. Hence, I was able to correct $100 \mathrm{~nm}$ beads in a depth of up to $\sim 80 \mu \mathrm{m}$ whereas $40 \mathrm{~nm}$ beads were only corrected in a depth of up to $\sim 50 \mu \mathrm{m}$. Weak fluorescence will not be the only challenge, because instead of static beads that I have been using in fixed brain slices, the structures are dynamic in the living brain. If the structure changes during the correction process the optimization is not reliable anymore. Also, if the structure that is corrected is larger than the field of view for correction, edge effects can influence the optimization.

Additionally, the signal to noise ratio will be lower, as background fluorescence is typically brighter in vivo. However, the background fluorescence has no impact on the $5 \%$-metric because it is not taken into account. If the structure is carefully chosen to be brightest in the center region of the field of view this is also true for the edge effects. 
Bleaching will not influence the optimization if one additional image is taken per aberration mode because then the single images can be corrected. Taking 65 confocal images ( 5 for spherical, $2 \times 10$ for astigmatism, coma and trefoil) however, can decrease the fluorescence so far, that not all corrections can be done in the same field of view. Since I use images of $1 \mu \mathrm{m} \times 1 \mu \mathrm{m}$ I could correct some of the aberrations on one spot of the sample and the others on another spot nearby.

Another aspect about in vivo imaging is, that focusing deep into tissue will reduce the intensity of the light in the focal spot, since the tissue will scatter parts of the light [71. Scattering will cause loss of intensity, which affects excitation and STED beam and therefore reduces the image intensity as well as the FWHM.

\subsection{Outlook}

Before adaptive optics can be used for STED microscopy in the living mouse, the following needs to be improved. The deformable mirror needs to be exchanged, as the power dependence hinders a good workflow and the additional aberrations add difficulties to adjusting the setup. As well it would be beneficial to use a model with more actuators, which are necessary when higher order aberrations are going to be corrected. Furthermore, it needs to be tested if an additional SLM in the STED beam can increase the aberration correction so far, that the more complicated setup and longer adjustment routine are justified. A SLM is absolutely necessary for 3D STED in the living mouse, as it will be used for creating the 3D STED PSF. Additionally the 3D PSF is more sensitive to aberrations than the 2D STED PSF and therefore the additional correction of the STED beam is needed. A beam scanner in the setup would increase imaging speed as well as the field of view. Furthermore, the possibility of injecting non-bleaching point like emitters into the mouse brain can be used for the correction, as it would make the correction easier. It has been shown that carbon-nanotubes as well as quantum dots can be injected into living tissue, as they have been used in in vivo experiments before [102, 103. In summary, the correction of aberrations in the living mouse will be a challenging task but the possibility of imaging deeper into the brain is worth the challenge. 


\section{Appendix}

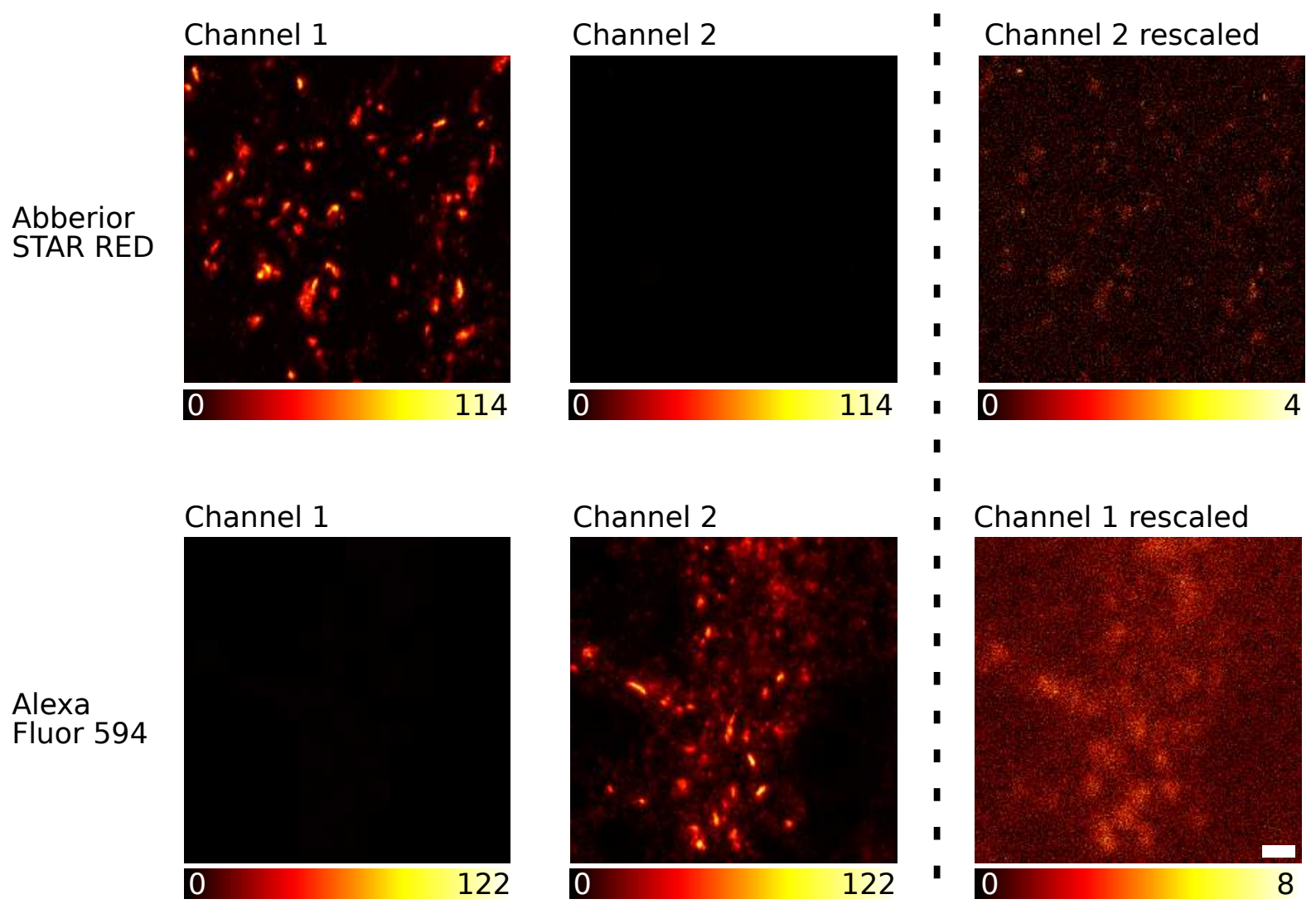

Figure 5.1: Single color STED images to visualize the channel crosstalk. The staining conditions were the same as in Figure 2.6. On the right side the intensity of the cross talk channel is rescaled to show that the crosstalk is negligible. The images were acquired with a pixel size of $30 \mathrm{~nm}$ and $50 \mu$ s pixel dwell time. The laser powers were $\sim 7 \mu \mathrm{W}$ for excitation I, $\sim 13 \mu \mathrm{W}$ for excitation II and $260 \mathrm{~mW}$ for the STED laser. Scalebar: 500nm 
a) Alexa Fluor 594
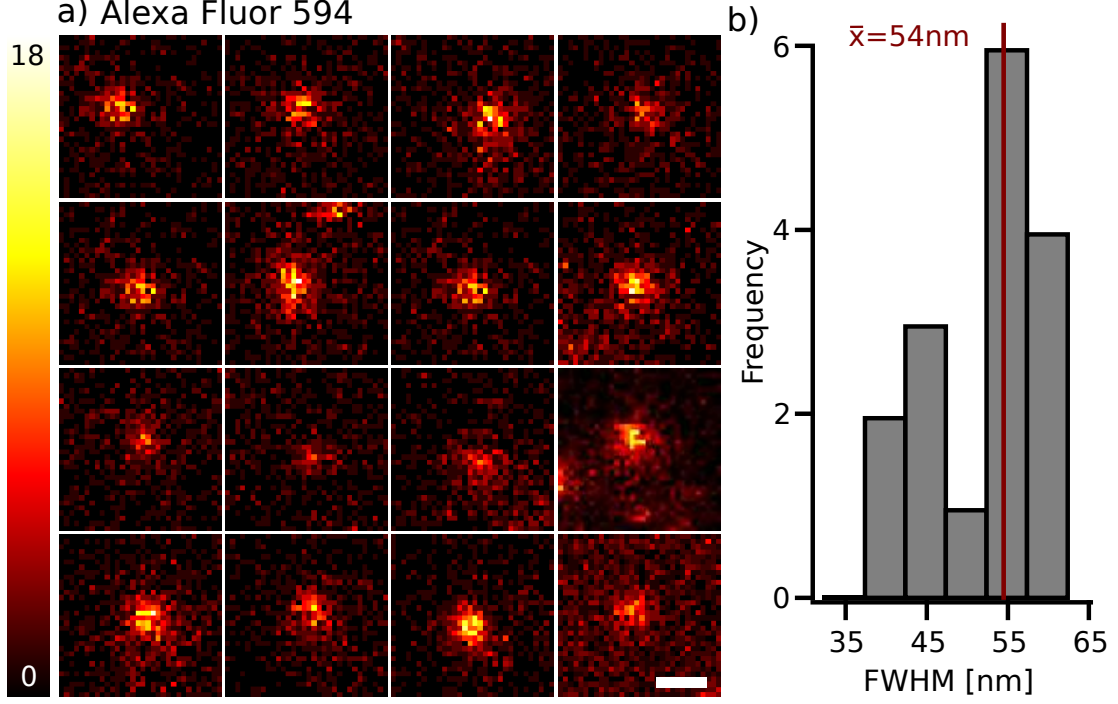

c) STAR RED

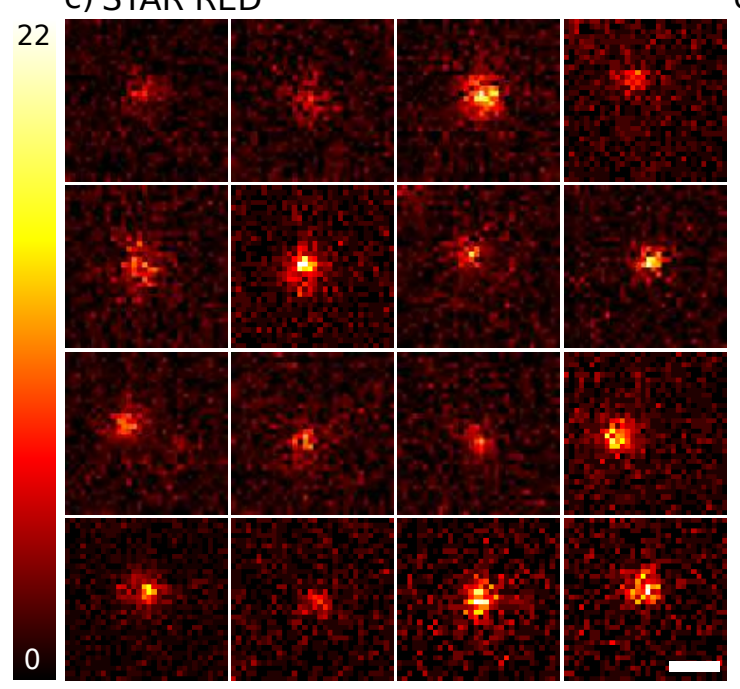

d)

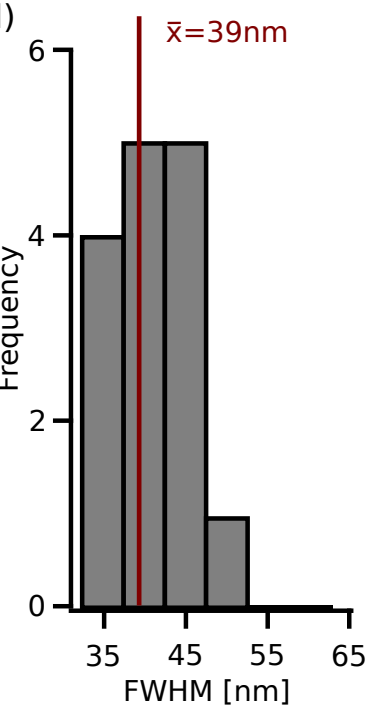

Figure 5.2: Measuring the resolution in a two-color image by selecting single primary plus secondary antibodies in the background of immunolabeled cells and determining the FWHM. The FWHM was determined by fitting a Lorentzian function to the line profile ( 3 pixels wide) of the point-like structures in x-direction. Done for a) Alexa Fluor 594 and c) STAR RED. b) and d) show the histograms of the measured FWHMs and their average. The images were acquired with a pixel size of $10 \mathrm{~nm}$ and $50 \mu$ s pixel dwell time. The laser powers were $\sim 12 \mu \mathrm{W}$ for excitation I, $\sim 20 \mu \mathrm{W}$ for excitation II and $260 \mathrm{~mW}$ for the STED laser. Scalebar: $100 \mathrm{~nm}$ 


\section{Bibliography}

[1] British Museum The Nimrud Lens / The Layard Lens. http://www.britishmuseum.org accessed 2018-10-09.

[2] David C. Lindberg. Theories of vision from al-Kindi to Kepler. University of Chicago Press, 1976.

[3] Henry C. King. The history of the telescope. Dover Publications, 1979.

[4] Roshdi Rashed. A Pioneer in Anaclastics: Ibn Sahl on Burning Mirrors and Lenses. 81(3):464-491, 2017.

[5] Kilby, Jack and Robert Noyce. Inventors and Inventions. Marshall Cavendish Coporation, volume 4 edition, 2008.

[6] Savile. Bradbury. The evolution of the microscope. Pergamon Press, New York, 1967.

[7] B. Matrin. New Elements of Optics; Or, the Theory of the Aberrations, Dissipation, and Colours of Light. Gale Ecco, Print Editions, 1759.

[8] Lisa. Yount. Antoni van Leeuwenhoek: first to see microscopic life. Enslow Publishers, 2008.

[9] Emil Born, Max, Wolf. Principles of Optics. Cambridge University Press, 7 edition, 1980.

[10] H. Volkmann. Ernst Abbe and His Work. Applied Optics, 5(11):1720, 1966.

[11] Brad Amos, G. McConnell, and Tony Wilson. Confocal Microscopy. In Comprehensive Biophysics, chapter 2.2, pages 3-23. Academic Press, 2012.

[12] C. R. Burch and J. P. P. Stock. Phase-Contrast Microscopy. Journal of Scientific Instruments, 19(5):71-75, may 1942.

[13] G.G. Stokes. On the Change of Refrangibility of Light. Royal Society, 142(1852):463-562, 2016.

[14] Joseph R Lakowicz. Principles of fluorescence spectroscopy. In Principles of Fluorescence Spectroscopy, pages 529-569. Springer US, third edit edition, 1999. 
[15] Shlomo Pundak and Rodney S. Roche. Tyrosine and tyrosinate fluorescence of bovine testes calmodulin: calcium and $\mathrm{pH}$ dependence. Biochemistry, 23(7):15491555, mar 1984.

[16] Elizabeth A. Jares-Erijman and Thomas M. Jovin. FRET imaging. Nature Biotechnology, 21(11):1387-1395, 2003.

[17] Sarah Rice, Abel W. Lin, Daniel Safer, Cynthia L. Hart, Nariman Naber, Bridget O. Carragher, Shane M. Cain, Elena Pechatnikova, Elizabeth M. Wilson-Kubalek, Michael Whittaker, Edward Pate, Roger Cooke, Edwin W. Taylor, Ronald A. Milligan, and Ronald D. Vale. A structural change in the kinesin motor protein that drives motility. Nature, 402(6763):778-784, dec 1999.

[18] Daisuke Kajihara, Ryoji Abe, Issei Iijima, Chie Komiyama, Masahiko Sisido, and Takahiro Hohsaka. FRET analysis of protein conformational change through position-specific incorporation of fluorescent amino acids. Nature Methods, 3(11):923-929, nov 2006.

[19] Lars Kastrup. Fluorescence Depletion by Stimulated Emission in Single-Molecule Spectroscopy. PhD thesis, Ruperto-Carola University of Heidelberg, 2004.

[20] E. Rittweger, B. R. Rankin, V. Westphal, and S. W. Hell. Fluorescence depletion mechanisms in super-resolving STED microscopy. Chemical Physics Letters, 442(46):483-487, 2007.

[21] Benjamin Harke, Jan Keller, Chaitanya K. Ullal, Volker Westphal, Andreas Schönle, and Stefan W. Hell. Resolution scaling in STED microscopy. Optical Society of America, 16(6):1347-1355, 2008.

[22] Dominik Wildanger, Brian R. Patton, Heiko Schill, Luca Marseglia, J. P. Hadden, Sebastian Knauer, Andreas Schönle, John G. Rarity, Jeremy L. O’Brien, Stefan W. Hell, and Jason M. Smith. Solid immersion facilitates fluorescence microscopy with nanometer resolution and sub-Ångström emitter localization. Advanced Materials, 24(44):309-313, 2012.

[23] Marcel Leutenegger, Christian Eggeling, and Stefan W. Hell. Analytical description of STED microscopy performance. Optics Express, 18(25):26417, 2010.

[24] Derek Huang, Henry Timmers, Adam Roberts, Niranjan Shivaram, and Arvinder S. Sandhu. A low-cost spatial light modulator for use in undergraduate and graduate optics labs. American Journal of Physics, 80(3):211-215, 2012.

[25] Pinaki Sarder and Arye Nehorai. Deconvolution methods for 3-D fluorescence microscopy images. IEEE Signal Processing Magazine, 23(3):32-45, 2006. 
[26] Jan Keller, Andreas Schönle, and Stefan W. Hell. Efficient fluorescence inhibition patterns for RESOLFT microscopy. Optics Express, 15(6):3361, 2007.

[27] Jennifer Lippincott-Schwartz, Eric Betzig, George H. Patterson, Rachid Sougrat, O. Wolf Lindwasser, Scott Olenych, Juan S. Bonifacino, Michael W. Davidson, and Harald F. Hess. Supplement: Imaging intracellular fluorescent proteins at nanometer resolution. Science (New York, N.Y.), 313(5793):1642-5, 2006.

[28] M.J. Rust, Mark Bates, and Xiaowei Zhuang. Stochastic optical reconstruction miscroscopy (STORM) provides sub-diffraction-limit image resolution. Nat. Methods, 3(10):793-795, 2006.

[29] Eric Betzig. Proposed method for molecular optical imaging. Optics letters, 20(3):237-9, 1995.

[30] Suliana Manley, Jennifer M. Gillette, George H. Patterson, Hari Shroff, Harald F. Hess, Eric Betzig, and Jennifer Lippincott-Schwartz. High-density mapping of single-molecule trajectories with photoactivated localization microscopy. Nature Methods, 5(2):155-157, 2008.

[31] A. Sharonov and R. M. Hochstrasser. Wide-field subdiffraction imaging by accumulated binding of diffusing probes. Proceedings of the National Academy of Sciences, 103(50):18911-18916, 2006.

[32] Jonas Fölling, Mariano Bossi, Hannes Bock, Rebecca Medda, Christian A. Wurm, Birka Hein, Stefan Jakobs, Christian Eggeling, and Stefan W. Hell. Fluorescence nanoscopy by ground-state depletion and single-molecule return. Nature Methods, $5(11): 943-945,2008$.

[33] Francisco Balzarotti, Yvan Eilers, Klaus C Gwosch, Arvid H Gynnå, Volker Westphal, Fernando D Stefani, Johan Elf, and Stefan W Hell. With Minimal Photon Fluxes. Science, 355(February):606-612, 2017.

[34] Jennifer-Magdalena Masch. STED nanoscopy of synaptic substructures in living mice. Phd thesis, Georg-August-Universität Göttingen, 2017.

[35] Steen J. Madsen. Optical methods and instrumentation in brain imaging and therapy. Springer Science, New York, 2013.

[36] Jörn Heine, Matthias Reuss, Benjamin Harke, Elisa D'Este, Steffen J. Sahl, and Stefan W. Hell. Adaptive-illumination STED nanoscopy. Proceedings of the National Academy of Sciences, 114(37):9797-9802, sep 2017.

[37] U. Valentin Nägerl, Katrin I. Willig, Birka Hein, Stefan W. Hell, and Tobias Bonhoeffer. Live-cell imaging of dendritic spines by STED microscopy. PNAS, 105(48):18982-7, 2008. 
[38] Katrin I. Willig, Silvio O. Rizzoli, Volker Westphal, Reinhard Jahn, and Stefan W. Hell. STED microscopy reveals that synaptotagmin remains clustered after synaptic vesicle exocytosis. Nature, 440(7086):935-939, 2006.

[39] Nicolai T. Urban, Katrin I. Willig, Stefan W. Hell, and U. Valentin Nägerl. STED Nanoscopy of Actin Dynamics in Synapses Deep Inside Living Brain Slices. Biophysical Journal, 101(5):1277-1284, sep 2011.

[40] Elisa D'Este, Dirk Kamin, Fabian Göttfert, Ahmed El-Hady, and Stefan W. Hell. STED Nanoscopy Reveals the Ubiquity of Subcortical Cytoskeleton Periodicity in Living Neurons. Cell Reports, 10(8):1246-1251, mar 2015.

[41] B. Harke. 3D STED microscopy with pulsed and continuous wave lasers. Phd thesis, Georg-August-Universität Göttingen, 2008.

[42] Bo Huang, Sara A. Jones, Boerries Brandenburg, and Xiaowei Zhuang. Whole-cell 3D STORM reveals interactions between cellular structures with nanometer-scale resolution. Nature Methods, 5(12):1047-1052, dec 2008.

[43] Zhiping Zeng and Peng Xi. Advances in three-dimensional super-resolution nanoscopy. Microscopy Research and Technique, 79(10):893-898, oct 2016.

[44] Martin J. Booth. Adaptive optical microscopy: The ongoing quest for a perfect image. Light: Science and Applications, 3(November 2013):1-7, 2014.

[45] Martin Booth, Débora Andrade, Daniel Burke, Brian Patton, and Mantas Zurauskas. Aberrations and adaptive optics in super-resolution microscopy. $\mathrm{Mi}$ croscopy, 64(4):251-261, aug 2015.

[46] H. W. Babcock. The Possibility of Compensating Astronomical Seeing. Publications of the Astronomical Society of the Pacific, 65(386):229, 1953.

[47] John W. Hardy. Adaptive optics for astronomical telescopes. Oxford University Press, 1998.

[48] Ben C. Platt and Roland Shack. History and Principles of Shack-Hartmann Wavefront Sensing. Journal of Refractive Surgery, 17(Sept/Oct):573-577, 2001.

[49] Daniel R. Neal, James Copland, and David A. Neal. Shack-Hartmann wavefront sensor precision and accuracy. SPIE., 4779:148, 2002.

[50] Francois Roddier. Adaptive Optics in Astronomy. Cambridge University Press, 1999.

[51] Martin J. Booth. Adaptive optics in microscopy. Philosophical Transactions of the Royal Society A: Mathematical, Physical and Engineering Sciences, 365(1861):28292843, 2007. 
[52] N. Ji, T. R. Sato, and E. Betzig. Characterization and adaptive optical correction of aberrations during in vivo imaging in the mouse cortex. Proceedings of the National Academy of Sciences, 109(1):22-27, 2012.

[53] P. Marsh, D. Burns, and J. Girkin. Practical implementation of adaptive optics in multiphoton microscopy. Optics Express, 11(10):1123, 2003.

[54] Wei Yan, Yanlong Yang, Yu Tan, Xun Chen, Yang Li, Junle Qu, and Tong Ye. Coherent optical adaptive technique improves the spatial resolution of STED microscopy in thick samples. Photonics Research, 5(3):176, 2017.

[55] Kai Wang, Wenzhi Sun, Christopher T. Richie, Brandon K. Harvey, Eric Betzig, and $\mathrm{Na}$ Ji. Direct wavefront sensing for high-resolution in vivo imaging in scattering tissue. Nature Communications, 6:1-6, 2015.

[56] Martin J. Booth. Adaptive Optics in Microscopy. In Optical and Digital Image Processing: Fundamentals and Applications, pages 295-322. Wiley, 2011.

[57] Jacopo Antonello, Daniel Burke, and Martin J. Booth. Aberrations in stimulated emission depletion (STED) microscopy. Optics Communications, 404:203-209, 2017.

[58] Wen Lo, Yen Sun, Sun-Jan Lin, Shiou-Hwa Jee, and Chen-Yuan Dong. Spherical aberration correction in multiphoton fluorescence imaging using objective correction collar. Journal of biomedical optics, 10(3):034006, 2005.

[59] A. M. Weiner. Femtosecond pulse shaping using spatial light modulators. Review of Scientific Instruments, 71(5):1929-1960, 2000.

[60] V. Boyer, R. M. Godun, G. Smirne, D. Cassettari, C. M. Chandrashekar, A B Deb, Z J Laczik, and C J Foot. Dynamic manipulation of Bose-Einstein condensates with a spatial light modulator. Physical Review A - Atomic, Molecular, and Optical Physics, 73(3):2-5, 2006.

[61] Hamamatsu. Phase spatial light modulator LCOS-SLM. In Opto-semiconductor handbook, chapter 12, pages 1-14. Hamamatsu Photonics, 2017.

[62] Martin O. Lenz, Hugo G. Sinclair, Alexander Savell, James H. Clegg, Alice C.N. Brown, Daniel M. Davis, Chris Dunsby, Mark A.A. Neil, and Paul M.W. French. 3-D stimulated emission depletion microscopy with programmable aberration correction. Journal of Biophotonics, 7(1-2):29-36, 2014.

[63] A. R. Jewel, V. Akondi, and B. Vohnsen. A direct comparison between a MEMS deformable mirror and a liquid crystal spatial light modulator in signal-based wavefront sensing. Journal of the European Optical Society, 8(October), 2013. 
[64] Travis J. Gould, Daniel Burke, Joerg Bewersdorf, and Martin J. Booth. Adaptive optics enables 3D STED microscopy in aberrating specimens. Optics Express, 20(19):20998, 2012.

[65] Brian R. Patton, Daniel Burke, David Owald, Travis J. Gould, Joerg Bewersdorf, and Martin J. Booth. Three-dimensional STED microscopy of aberrating tissue using dual adaptive optics. Optics Express, 24(8):8862, 2016.

[66] Travis J. Gould, Emil B. Kromann, Daniel Burke, Martin J. Booth, and Joerg Bewersdorf. Auto-aligning stimulated emission depletion microscope using adaptive optics. Optics Letters, 38(11):1860, 2013.

[67] P. Kner, J. W. Sedat, D. A. Agard, and Z. Kam. High-resolution wide-field microscopy with adaptive optics for spherical aberration correction and motionless focusing. Journal of Microscopy, 237(2):136-147, feb 2010.

[68] Sebastian Berning, Katrin I. Willig, Heinz Steffens, Payam Dibaj, and Stefan W. Hell. Nanoscopy in a living mouse brain. Science, 335(6068):551, 2012.

[69] Katrin I. Willig, Heinz Steffens, Carola Gregor, Alexander Herholt, Moritz J. Rossner, and Stefan W. Hell. Nanoscopy of filamentous actin in cortical dendrites of a living mouse. Biophysical Journal, 106(1):2013-2015, 2014.

[70] Md. Islam, Masato Honma, Takakazu Nakabayashi, Masataka Kinjo, Nobuhiro Ohta, Md. Serajul Islam, Masato Honma, Takakazu Nakabayashi, Masataka Kinjo, and Nobuhiro Ohta. pH Dependence of the Fluorescence Lifetime of FAD in Solution and in Cells. International Journal of Molecular Sciences, 14(1):1952-1963, jan 2013.

[71] Saif I. Al-Juboori, Anna Dondzillo, Elizabeth A. Stubblefield, Gidon Felsen, Tim C. Lei, and Achim Klug. Light Scattering Properties Vary across Different Regions of the Adult Mouse Brain. PLoS ONE, 8(7):1-9, 2013.

[72] Vasilis Ntziachristos. Going deeper than microscopy: The optical imaging frontier in biology. Nature Methods, 7(8):603-614, aug 2010.

[73] Fabian Göttfert, Christian A. Wurm, Veronika Mueller, Sebastian Berning, Volker C. Cordes, Alf Honigmann, and Stefan W. Hell. Coaligned dual-channel STED nanoscopy and molecular diffusion analysis at $20 \mathrm{~nm}$ resolution. Biophysical Journal, 105(1):L01-L03, jul 2013.

[74] Kristjan R. Jessen and Rhona Mirsky. The origin and development of glial cells in peripheral nerves. Nature Reviews Neuroscience, 6(9):671-682, 2005. 
[75] Martin Chalfie, Yuan Tu, Ghia Euskirchen, William W. Ward, and Douglas C. Prashert. Green Fluorescent Protein as a Marker for Gene Expression. Science (New York, N.Y.), 263(5148):802-805, 2009.

[76] Waja Wegner, Peter Ilgen, Carola Gregor, Joris Van Dort, Alexander C. Mott, Heinz Steffens, and Katrin I. Willig. In vivo mouse and live cell STED microscopy of neuronal actin plasticity using far-red emitting fluorescent proteins. Scientific Reports, 7(1):1-10, 2017.

[77] Jun Chu, Russell D. Haynes, Stéphane Y. Corbel, Pengpeng Li, Emilio GonzálezGonzález, John S. Burg, Niloufar J. Ataie, Amy J. Lam, Paula J. Cranfill, Michelle A. Baird, Michael W. Davidson, Ho Leung Ng, K Christopher Garcia, Christopher H. Contag, Kang Shen, Helen M. Blau, and Michael Z. Lin. Noninvasive intravital imaging of cellular differentiation with a bright red-excitable fluorescent protein. Nature Methods, 11(5):572-578, may 2014.

[78] Thomas C. Südhof. The presynaptic active zone. Neuron, 75(1):11-25, jul 2012.

[79] Cecile Bats, Laurent Groc, and Daniel Choquet. The Interaction between Stargazin and PSD-95 Regulates AMPA Receptor Surface Trafficking. Neuron, 53(5):719-734, mar 2007.

[80] Jennifer D. Petersen, Xiaobing Chen, Lucia Vinade, Ayse Dosemeci, John E. Lisman, and Thomas S. Reese. Distribution of postsynaptic density (PSD)-95 and $\mathrm{Ca} 2+/$ calmodulin-dependent protein kinase II at the PSD. The Journal of Neuroscience, 23(35):11270-11278, 2003.

[81] Waja Wegner, Alexander C. Mott, Seth G.N. Grant, Heinz Steffens, and Katrin I. Willig. In vivo STED microscopy visualizes PSD95 sub-structures and morphological changes over several hours in the mouse visual cortex. Scientific Reports, $8(1): 1-11,2018$.

[82] Johannes Schindelin, Ignacio Arganda-Carreras, Erwin Frise, Verena Kaynig, Mark Longair, Tobias Pietzsch, Stephan Preibisch, Curtis Rueden, Stephan Saalfeld, Benjamin Schmid, Jean-Yves Tinevez, Daniel James White, Volker Hartenstein, Kevin Eliceiri, Pavel Tomancak, and Albert Cardona. Fiji: an open-source platform for biological-image analysis. Nature Methods, 9(7):676-682, jul 2012.

[83] Stephen W. Paddock. Principles and Practices of Laser Scanning Confocal Microscopy. Molecular Biotechnology, 16(2):127-150, 2000.

[84] F. Zernike. Diffraction theory of the knife-edge test and its improved form, the phase-contrast method. Royal Astronomical Society, Mar:377-384, 1934. 
[85] Vasudevan lakshminarayanana. Zernikepolynomials: A guide. Journal of Modern Optics, 58(7):545-561, 2011.

[86] Robert J. Noll. Zernike polynomials and atmospheric turbulence*. Journal of the Optical Society of America, 66(3):207, 1976.

[87] Larry N. Thibos. Principles of Hartmann-Shack Aberrometry. Journal of Refractive Surgery, 16(October):1-3, 2000.

[88] Emmanuel Beaurepaire and D. Delphine. Accuracy of correction in modal sensorless adaptive optics. Optical Society of America, 20(3):18209-18219, 2012.

[89] Anisha Thayil and Martin J. Booth. Self calibration of sensorless adaptive optical microscopes. Journal of the European Optical Society, 6:16, 2011.

[90] Alexander Jesacher and Martin J. Booth. Sensorless adaptive optics for microscopy. In Adaptive Optics for Biological Imaging, page 79310G. CRC Press, 2013.

[91] Delphine Débarre, Edward J. Botcherby, Tomoko Watanabe, Shankar Srinivas, Martin J. Booth, and Tony Wilson. Image-based adaptive optics for two-photon microscopy. Optics Letters, 34(16):2495, 2009.

[92] L. P. Murray, J. C. Dainty, J. Coignus, and F. Felberer. Wavefront correction of extended objects through image sharpness maximisation. SPIE., 5823:60181A, 2005.

[93] Delphine Debarre, Martin J. Booth, and Tony Wilson. Image based adaptive optics through optimisation of low spatial frequencies. Optics Express, 15(13):8176, jun 2007.

[94] Python Software Foundation. www.Python.org.

[95] Phyton software package Specpy. https://pypi.org/project/specpy/.

[96] Sarah Frisken Gibson and Frederick Lanni. Experimental test of an analytical model of aberration in an oil-immersion objective lens used in three-dimensional light microscopy. Journal of the Optical Society of America A, 9(1):154, jan 1992.

[97] Fabian Göttfert. STED Microscopy with Scanning Fields Below the Diffraction Limit. Phd thesis, Georg-August-Universität Göttingen, 2015.

[98] Frederik Görlitz, Stina Guldbrand, Timothy H. Runcorn, Robert T. Murray, Angel L. Jaso-Tamame, Hugo G. Sinclair, Enrique Martinez-Perez, James R. Taylor, Mark A. A. Neil, Christopher Dunsby, and Paul M. W. French. easySLM-STED: Stimulated emission depletion microscopy with aberration correction, extended field of view and multiple beam scanning. Journal of Biophotonics, page e201800087, jul 2018. 
[99] Pit Bingen, Matthias Reuss, Johann Engelhardt, and Stefan W. Hell. Parallelized STED fluorescence nanoscopy. Optics Express, 19(24):23716, nov 2011.

[100] Yanghui Li, Hui Zhou, Xiaoyu Liu, Yuxue Li, and Le Wang. Effects of aberrations on effective point spread function in STED microscopy. Applied Optics, 57(15):4164, may 2018.

[101] Stefan W. Hell Sebastian Berning, Katrin I. Willig, Heinz Steffens, Payam Dibaj. Nanoscopy in a Living Mouse Brain. Science, 316(5828):1153-1158, may 2007.

[102] Zhuang Liu, Scott Tabakman, Kevin Welsher, and Hongjie Dai. Carbon nanotubes in biology and medicine: In vitro and in vivo detection, imaging and drug delivery. Nano Research, 2(2):85-120, 2009.

[103] S. S. Gambhir and S. Weiss. Quantum Dots for Live Cells. Science, 307(January):538-545, 2005. 


\section{Acknowledgments}

Writing a scientific thesis is not the work of just one person. Many people have helped me over the years and made it possible for me to achieve my goal and I am very grateful to everyone who contributed. Therefore I would like to thank the following people:

First of all I would like to thank Katrin Willig for giving me the chance to work in her group and providing me with everything I needed. Proposing the project, showing me the world of superresolution, leaving me a lot of space and time to make my own decisions and always being there to help with new ideas, discussions and encouraging me to finish my research. Christoph Schmidt for the interest in my thesis and ideas for further research. Stefan Hell for being part of my Thesis Committee. The members of my examination board for the time they spend, even though they could have spend it preparing for Christmas instead.

I would like to thank Waja Wegner for making my time in the lab even more enjoyable and helping wherever she could. It was always a pleasure when you could provide me with new samples, whether it were biological samples or cake. Also for the tips about labwork and skiing as well as for letting me "lend" the scotch tape and other things. Jannik Heise, Antonia Müller, Valérie Clavet-Fournier and Alexander Charles Mott for the shared time in the office and discussions about political and not so political topics. I learned a lot from you! Furthermore I want to thank Heinz Steffens and Peter Ilgen for the nice work atmosphere and the help they provided throughout my thesis. I want to thank Jan Keller for the software support and suppling the simulation routine.

Without the many custom made parts of the mechanical workshop, it would not have been possible to build the setups, so a big thank you for the help and optimizations of the parts. The software support is equality as important, so thank you Lothar Demel and Rolf Merker for always being available on short notice. I would like to thank all of the people I collaborated with for explaining the biology behind their research (more than once) and for the time they invested to create the perfect samples for imaging.

I want to thank my friends and family for the support and help they provided in any regard. I especially thank Waja Wegner, Maurice Seer and Alexander Charles Mott for proofreading, they eliminated lost of spelling mistakes and helped me to improve the logical structure my sentences were in.

Last and most I want to thank my wife Julia for everything; the sacrifices, the mental support, always being there for me and believing in me when I couldn't. Ik hou van jou! 